Foundations and Trends ${ }^{\circledR}$ in

Human-Computer Interaction

Vol. 3, Nos. 1-2 (2009) 1-137

(C) 2010 O. Shaer and E. Hornecker

DOI: $10.1561 / 1100000026$

\title{
Tangible User Interfaces: Past, Present, and Future Directions
}

\author{
By Orit Shaer and Eva Hornecker
}

\section{Contents}

1 Introduction

2 Origins of Tangible User Interfaces 6

2.1 Graspable User Interface $\quad 7$

2.2 Tangible Bits 8

2.3 Precursors of Tangible User Interfaces 10

3 Tangible Interfaces in a Broader Context 14

3.1 Related Research Areas 14

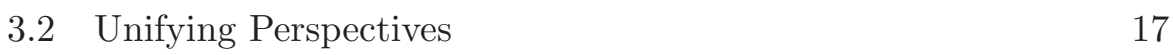

$\begin{array}{lll}3.3 & \text { Reality-Based Interaction } & 19\end{array}$

4 Application Domains $\quad 22$

4.1 TUIs for Learning 23

4.2 Problem Solving and Planning $\quad 27$

4.3 Information Visualization 31

4.4 Tangible Programming 33

4.5 Entertainment, Play, and Edutainment 36

$\begin{array}{lll}4.6 & \text { Music and Performance } & 39\end{array}$ 
4.7 Social Communication 43

4.8 Tangible Reminders and Tags 44

5 Frameworks and Taxonomies 46

5.1 Properties of Graspable User Interfaces 47

5.2 Conceptualization of TUIs and the MCRit

Interaction Model 48

5.3 Classifications of TUIs 49

5.4 Frameworks on Mappings: Coupling the Physical

5.5 Tokens and Constraints 54

5.6 Frameworks for Tangible and Sensor-Based Interaction 56

5.7 Domain-Specific Frameworks 59

6 Conceptual Foundations $\quad 62$

6.1 Cuing Interaction: Affordances, Constraints, Mappings and Image Schemas 62

6.2 Embodiment and Phenomenology 64

6.3 External Representation and Distributed Cognition 66

6.4 Two-Handed Interaction $\quad 69$

$\begin{array}{lll}6.5 \text { Semiotics } & 70\end{array}$

7 Implementation Technologies $\quad 73$

$\begin{array}{lll}7.1 & \text { RFID } & 74\end{array}$

$\begin{array}{lll}7.2 & \text { Computer Vision } & 75\end{array}$

$\begin{array}{lll}7.3 & \text { Microcontrollers, Sensors, and Actuators } & 77\end{array}$

7.4 Comparison of Implementation Technologies 79

7.5 Tool Support for Tangible Interaction 81

8 Design and Evaluation Methods $\quad 88$

8.1 Design and Implementation $\quad 88$

$\begin{array}{lll}8.2 \text { Evaluation } & 93\end{array}$ 
9 Strengths and Limitations of Tangible User Interfaces $\quad 96$

$\begin{array}{llr}9.1 & \text { Strengths } & 97\end{array}$

$\begin{array}{lll}9.2 \text { Limitations } & 105\end{array}$

10 Research Directions 109

$\begin{array}{ll}10.1 \text { Actuation } & 109\end{array}$

10.2 From Tangible User Interfaces to Organic User Interfaces 111

10.3 From Tangible Representation to Tangible Resources for Action

10.4 Whole-Body Interaction and Performative Tangible Interaction 114

$\begin{array}{ll}10.5 \text { Aesthetics } & 115\end{array}$

10.6 Long-Term Interaction Studies 115

11 Summary 118

$\begin{array}{ll}\text { Acknowledgments } & 120\end{array}$

$\begin{array}{ll}\text { References } & 121\end{array}$ 
Foundations and Trends ${ }^{\circledR}$ in

Human-Computer Interaction

Vol. 3, Nos. 1-2 (2009) 1-137

(C) 2010 O. Shaer and E. Hornecker

DOI: $10.1561 / 1100000026$

\title{
Tangible User Interfaces: Past, Present, and Future Directions
}

\section{Orit Shaer ${ }^{1}$ and Eva Hornecker ${ }^{2}$}

1 Wellesley College, 106 Central St., Wellesley, MA, 02481, USA, oshaer@wellesley.edu

2 University of Strathclyde, 26 Richmond Street, Glasgow, Scotland, G11XH,UK, eva@ehornecker.de

\begin{abstract}
In the last two decades, Tangible User Interfaces (TUIs) have emerged as a new interface type that interlinks the digital and physical worlds. Drawing upon users' knowledge and skills of interaction with the real non-digital world, TUIs show a potential to enhance the way in which people interact with and leverage digital information. However, TUI research is still in its infancy and extensive research is required in order to fully understand the implications of tangible user interfaces, to develop technologies that further bridge the digital and the physical, and to guide TUI design with empirical knowledge.

This monograph examines the existing body of work on Tangible User Interfaces. We start by sketching the history of tangible user interfaces, examining the intellectual origins of this field. We then present TUIs in a broader context, survey application domains, and review frameworks and taxonomies. We also discuss conceptual foundations
\end{abstract}


of TUIs including perspectives from cognitive sciences, psychology, and philosophy. Methods and technologies for designing, building, and evaluating TUIs are also addressed. Finally, we discuss the strengths and limitations of TUIs and chart directions for future research. 


\section{1}

\section{Introduction}

"We live in a complex world, filled with myriad objects, tools, toys, and people. Our lives are spent in diverse interaction with this environment. Yet, for the most part, our computing takes place sitting in front of, and staring at, a single glowing screen attached to an array of buttons and a mouse." [253]

For a long time, it seemed as if the human-computer interface was to be limited to working on a desktop computer, using a mouse and a keyboard to interact with windows, icons, menus, and pointers (WIMP). While the detailed design was being refined with ever more polished graphics, WIMP interfaces seemed undisputed and no alternative interaction styles existed. For any application domain, from productivity tools to games, the same generic input devices were employed.

Over the past two decades, human-computer interaction (HCI) researchers have developed a wide range of interaction styles and interfaces that diverge from the WIMP interface. Technological advancements and a better understanding of the psychological and social aspects of HCI have lead to a recent explosion of new post-WIMP 
interaction styles. Novel input devices that draw on users' skill of interaction with the real non-digital world gain increasing popularity (e.g., the Wii Remote controller, multi-touch surfaces). Simultaneously, an invisible revolution takes place: computers become embedded in everyday objects and environments, and products integrate computational and mechatronic components,

This monograph provides a survey of the research on Tangible User Interfaces (TUIs), an emerging post-WIMP interface type that is concerned with providing tangible representations to digital information and controls, allowing users to quite literally grasp data with their hands. Implemented using a variety of technologies and materials, TUIs computationally augment physical objects by coupling them to digital data. Serving as direct, tangible representations of digital information, these augmented physical objects often function as both input and output devices providing users with parallel feedback loops: physical, passive haptic feedback that informs users that a certain physical manipulation is complete; and digital, visual or auditory feedback that informs users of the computational interpretation of their action [237]. Interaction with TUIs is therefore not limited to the visual and aural senses, but also relies on the sense of touch. Furthermore, TUIs are not limited to two-dimensional images on a screen; interaction can become three-dimensional. Because TUIs are an emerging field of research, the design space of TUIs is constantly evolving. Thus, the goal of this monograph is not to bound what a TUI is or is not. Rather, it describes common characteristics of TUIs and discusses a range of perspectives so as to provide readers with means for thinking about particular designs.

Tangible Interfaces have an instant appeal to a broad range of users. They draw upon the human urge to be active and creative with one's hands [257], and can provide a means to interact with computational applications in ways that leverage users' knowledge and skills of interaction with the everyday, non-digital, world [119].

TUIs have become an established research area through the contributions of Hiroshi Ishii and his Tangible Media Group as well as through the efforts of other research groups worldwide. The word 'tangible' now appears in many calls for papers or conference session titles. 
Following diverse workshops related to tangible interfaces at different conferences, the first conference fully devoted to tangible interfaces and, more generally, tangible interaction, took place in 2007 in Baton Rouge, Louisiana. Since then, the annual TEI Conference (Tangible, Embedded and Embodied Interaction) serves as a focal point for a diverse community that consists of HCI researchers, technologists, product designers, artists, and others.

This monograph is the result of a systematic review of the body of work on tangible user interfaces. Our aim has been to provide a useful and unbiased overview of history, research trends, intellectual lineages, background theories, and technologies, and open research questions for anyone who wants to start working in this area, be it in developing systems or analyzing and evaluating them. We first surveyed seminal work on tangible user interfaces to expose lines of intellectual influence. Then, in order to clarify the scope of this monograph we examined past TEI and CHI proceedings for emerging themes. We then identified a set of questions to be answered by this monograph and conducted dedicated literature research on each of these questions.

We begin by sketching the history of tangible user interfaces, taking a look at the origins of this field. We then discuss the broader research context surrounding TUIs, which includes a range of related research areas. Section 4 is devoted to an overview of dominant application areas of TUIs. Section 5 provides an overview of frameworks and theoretical work in the field, discussing attempts to conceptualize, categorize, analyze, and describe TUIs, as well as analytical approaches to understand issues of TUI interaction. We then present conceptual foundations underlying the ideas of TUIs in Section 6. Section 7 provides an overview of implementation technologies and toolkits for building TUIs. We then move on to design and evaluation methods in Section 8. We close with a discussion of the strengths and limitations of TUIs and future research directions. 


\section{Origins of Tangible User Interfaces}

The development of the notion of a "tangible interface" is closely tied to the initial motivation for Augmented Reality and Ubiquitous Computing. In 1993, a special issue of the Communications of the ACM titled "Back to the Real World" [253] argued that both desktop computers and virtual reality estrange humans from their "natural environment". The issue suggested that rather than forcing users to enter a virtual world, one should augment and enrich the real world with digital functionality. This approach was motivated by the desire to retain the richness and situatedness of physical interaction, and by the attempt to embed computing in existing environments and human practices to enable fluid transitions between "the digital" and "the real". Ideas from ethnography, situated cognition, and phenomenology became influential in the argumentation for Augmented Reality and Ubiquitous Computing: "humans are of and in the everyday world" [251]. Tangible Interfaces emerged as part of this trend.

While underlying ideas for tangible user interfaces had been discussed in the "Back to the Real World" special issue, it took a few years for these ideas to evolve into an interaction style in its own right. In 1995, Fitzmaurice et al. [67] introduced the notion of a Graspable Interface, where graspable handles are used to manipulate digital objects. Ishii and his students [117] presented the more 
comprehensive vision of Tangible Bits in 1997. Their vision centered on turning the physical world into an interface by connecting objects and surfaces with digital data. Based on this work, the tangible user interface has emerged as a new interface and interaction style.

While Ishii and his students developed a rich research agenda to further investigate their Tangible Bits vision, other research teams focused on specific application domains and the support of established work practices through the augmentation of existing media and artifacts. Such efforts often resulted in systems that can also be classified as Tangible Interfaces. Particularly notable is the work of Wendy Mackay on the use of flight strips in air traffic control and on augmented paper in video storyboarding [150]. Similar ideas were developed simultaneously worldwide, indicating a felt need for a countermovement to the increasing digitization and virtualization. Examples include the German Real Reality approach for simultaneous building of real and digital models $[24,25]$, and the work of Rauterberg and his group in Switzerland. The latter extended Fitzmaurice's graspable interface idea and developed Build-IT, an augmented reality tabletop planning tool that is interacted via the principle of graspable handles. In Japan, Suzuki and Kato [230, 231] developed AlgoBlocks to support groups of children in learning to program. Cohen et al. [41] developed Logjam to support video logging and coding.

For most of the decade following the proposition of TUIs as a novel interface style, research focused on developing systems that explore technical possibilities. In recent years, this proof-of-concept phase has led on to a more mature stage of research with increased emphasis on conceptual design, user and field tests, critical reflection, theory, and building of design knowledge. Connections with related developments in the design disciplines became stronger, especially since a range of toolkits have become available which considerably lower the threshold for developing TUIs.

\subsection{Graspable User Interface}

In 1995, Fitzmaurice et al. [67] introduced the concept of a Graspable Interface, using wooden blocks as graspable handles to manipulate 
digital objects. Their aim was to increase the directness and manipulability of graphical user interfaces. A block is anchored to a graphical object on the monitor by placing it on top of it. Moving and rotating the block has the graphic object moving in synchrony. Placing two blocks on two corners of an object activates a zoom as the two corners will be dragged along with the blocks. This allowed for the kinds of two-handed or two-fingered interactions that we nowadays know from multi-touch surfaces. A further focus was the use of functionally dedicated input tools.

Graspable handles in combination with functionally dedicated input tools were argued to distribute input in space instead of time, effectively de-sequentializing interaction, to support bimanual action and to reduce the mediation between input devices and interaction objects. A system that directly builds on this idea is Rauterberg's Build-IT [69]. This utilizes said input mechanisms in combination with Augmented Reality visualizations for architectural and factory planning tasks.

\subsection{Tangible Bits}

Only a few years later, Hiroshi Ishii and his students introduced the notion of Tangible Bits which soon led to proposition of a Tangible User Interface [117]. The aim was to make bits directly accessible and manipulable, using the real world as a display and as medium for manipulation - the entire world could become an interface. Data could be connected with physical artifacts and architectonic surfaces, making bits tangible. Ambient displays on the other hand would represent information through sound, lights, air, or water movement. The artwork of Natalie Jeremijenko, in particular LiveWire, a dangling, dancing string hanging from the ceiling with its movement visualizing

network and website traffic served as an inspiration for the concept of ambient displays.

The change of term from graspable to tangible seems deliberate. Whereas "graspable" emphasizes the ability to manually manipulate objects, the meaning of "tangible" encompasses "realness/sureness", being able to be touched as well as the action of touching, which 
includes multisensory perception:

"GUIs fall short of embracing the richness of human senses and skills people have developed through a lifetime of interaction with the physical world. Our attempt is to change 'painted bits' into 'tangible bits' by taking advantage of multiple senses and the multimodality of human interactions with the real world. We believe the use of graspable objects and ambient media will lead us to a much richer multi-sensory experience of digital information." [117]

Ishii's work focused on using tangible objects to both manipulate and represent digital content. One of the first TUI prototypes was Tangible Geospace, an interactive map of the MIT Campus on a projection table. Placing physical icons onto the table, e.g., a plexiglas model of the MIT dome, had the map reposition itself so that the model was positioned over the respective building on the map. Adding another tangible model made the map zoom and turn to match the buildings. Small movable monitors served as a magic lens showing a 3D representation of the underlying area. These interfaces built on the graspable interface's interaction principle of bimanual direct manipulation, but replaced its abstract and generic blocks with iconic and symbolic stand-ins.

Still, the first TUI prototypes were influenced strongly from GUImetaphors. Later projects such as Urp [241] intentionally aimed to divert from GUI-like interaction, focusing on graspable tokens that serve for manipulating as well as representing data. Urp supports urban planning processes (see Figure 2.1). It enables users to interact with wind flow and sunlight simulations through the placement of physical building models and tools upon a surface. The tangible building models cast (digital) shadows that are projected onto the surface. Simulated wind flow is projected as lines onto the surface. Several tangible tools enable users to control and alter the urban model. For example, users can probe the wind speed or distances, change the material properties of buildings (glass or stone walls), and change the time of day. Such 


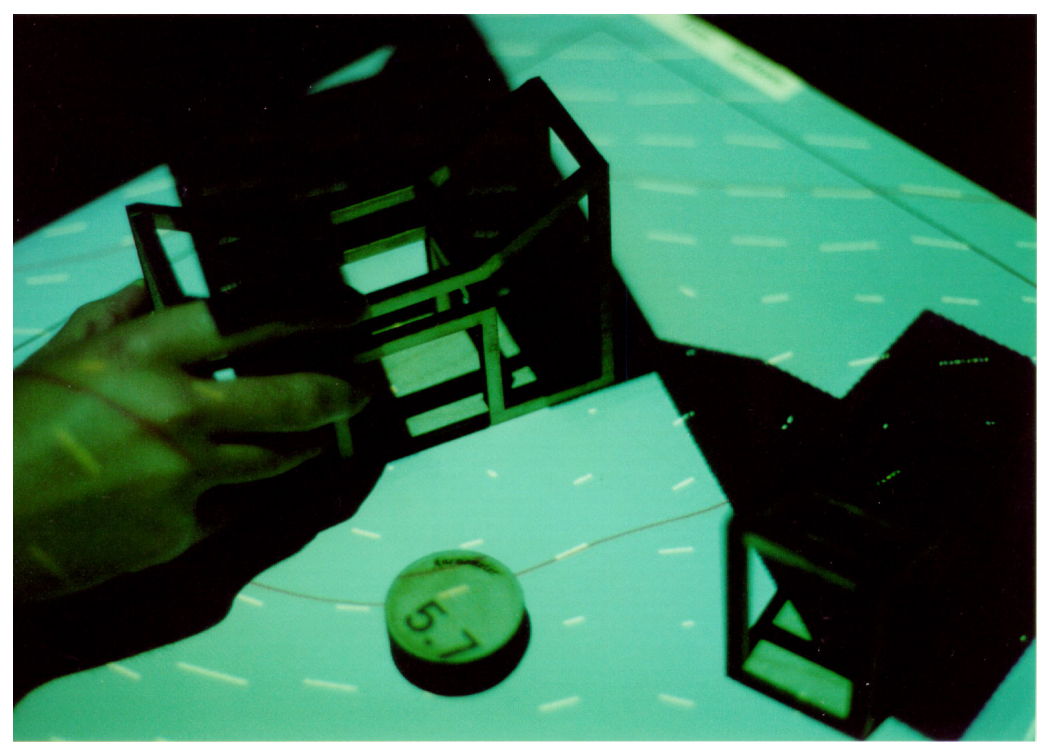

Fig. 2.1 Urp [241], a TUI for urban planning that combines physical models with interactive simualation. Projections show the flow of wind, and a wind probe (the circular object) is used to investigate wind speed (photo: by E. Hornecker).

changes affect the digital shadows that are projected and the wind simulation.

\subsection{Precursors of Tangible User Interfaces}

Several precursors to the work of Ishii and his students have influenced the field. These addressed issues in specific application domains such as architecture, product design, and educational technology. The ideas introduced by these systems later inspired HCI researchers in their pursuit to develop new interface and interaction concepts.

\subsubsection{The Slot Machine}

Probably the first system that can be classified as a tangible interface was Perlman's Slot Machine [185]. The Slot Machine uses physical cards to represent language constructs that are used to program the Logo Turtle (see also [161]). Seymour Papert's research had shown that while the physical turtle robot helped children to understand how geometric 
forms are created in space, writing programs was difficult for younger children and impossible for preschoolers who could not type. Perlman believed that these difficulties result not only from the language syntax, but also from the user interface. Her first prototype consisted of a box with a set of buttons that allowed devising simple programs from actions and numbers. The box then was used as a remote control for the turtle. This device could also record and replay the turtle movement, providing a programming-by-demonstration mode. Her final prototype was the Slot Machine, which allowed modifying programs and procedure calls.

In the Slot Machine, each programming language construct (an action, number, variable, or condition) is represented by a plastic card. To specify a program, sequences of cards are inserted into one of three differently colored racks on the machine. On the left of the rack is a "Do It" button, that causes the turtle to execute the commands from left to right. Stacking cards of different type onto each other creates complex commands such as "move forward twice". Placing a special colored card in a rack invokes a procedure call for the respectively colored rack that upon execution returns to the remainder of the rack. This mechanism implements function calls as well as simple recursion.

\subsubsection{The Marble Answering Machine}

Often mentioned as inspiration for the development of tangible interfaces [117] are the works of product designer Durrell Bishop. During his studies at the Royal College of Art, Bishop designed the Marble Answering Machine as a concept sketch $[1,190]$. In the Marble Answering Machine, incoming calls are represented with colored marbles that roll into a bowl embedded in the machine (see Figure 2.2). Placed into an indentation, the messages are played back. Putting a marble onto an indentation on the phone calls the number from which the call originated.

Bishop's designs rely on physical affordances and users' everyday knowledge to communicate the functionality and the how to interact [1]. These ideas were very different to the dominant school of product design in the 1990s, which employed product semantics primarily to influence 

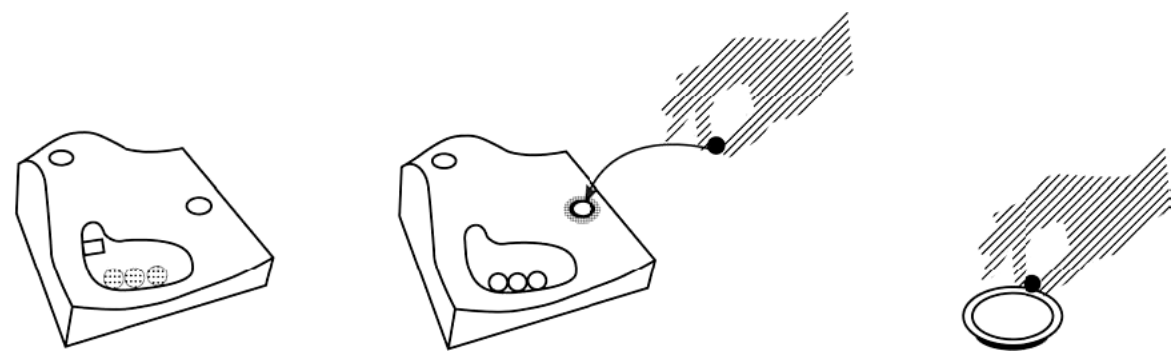

Fig. 2.2 The Marble Answering Machine [1]. Left: new messages have arrived and the user chooses to keepsake one to hear later. Right: the user plays back the selected message (graphics by Yvonne Baier, reprinted from form+zweck No. 22 www.formundzweck.de).

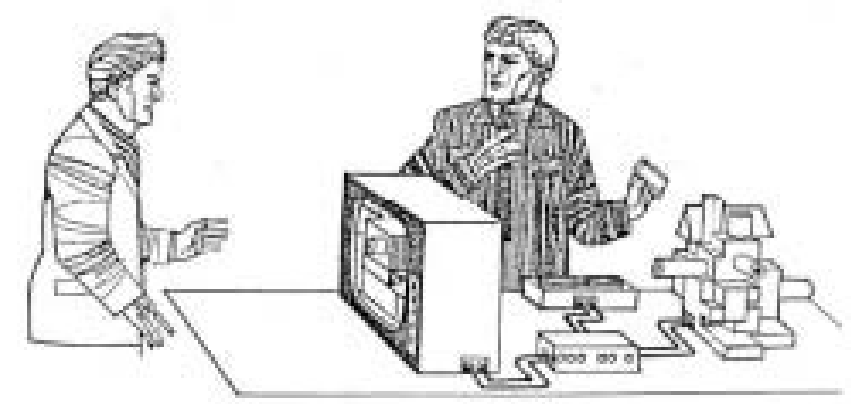

Fig. 2.3 Frazer and Frazer [71] envisioned an intelligent 3D modeling system that creates a virtual model from tangible manipulation (graphic courtesy: John Frazer).

users' emotions and associations. Most striking is how Bishop's works assign new meanings to objects (object mapping), turning them into pointers to something else, into containers for data and references to other objects in a network. Many of his designs further employ spatial mappings, deriving meaning from the context of an action (e.g., its place). Bishop's designs use known objects as legible references to the aesthetics of new electronic projects, yet they refrain from simplistic literal metaphors. Playfully recombining meanings and actions, Bishop's designs have remained a challenge and inspiration.

\subsubsection{Intelligent 3D Modeling}

In the early 1980s, independently of each other, both Robert Aish $[3,4]$ and the team around John Frazer $[70,71,72]$ were looking for 
alternatives to architectural CAD systems which at that time were clunky and cumbersome. These two groups were motivated by similar ideas. They sought to enable the future inhabitants of buildings to partake in design discussions with architects, to simplify the "manmachine dialog" with CAD, and to support rapid idea testing.

Thus, both came up with the idea of using physical models as input devices for CAD systems. Aish described his approach in 1979 [3], arguing that numerical CAD-modeling languages discourage rapid testing and alteration of ideas. Frazer was then first to build a working prototype, demoed live at the Computer Graphics conference in 1980. Aish and Frazer both developed systems for "3D modelling" where users build a physical model from provided blocks. The computer then interrogates or scans the assembly, deduces location, orientation and type of each component, and creates a digital model. Users can configure the digital properties of blocks and let the computer perform calculations such as floor space, water piping, or energy consumption. The underlying computer simulation could also provide suggestions on how to improve the design. Once the user is satisfied, the machine can produce the plans and working drawings.

Frazer's team (for an overview see [70]) experimented with a variety of application areas and systems, some based on components that could be plugged onto a $2 \mathrm{D}$ grid, others based on building blocks that could be connected to 3D structures. The blocks had internal circuitry, being able to scan its connections, poll its neighbours, and to pass messages. By 1982 the system was miniaturized to bricks smaller than two sugar cubes. Aish, on the other hand, experimented with a truly bi-directional human-machine dialog [4], using a robot to execute the computer's suggestions for changing the physical model. 


\section{Tangible Interfaces in a Broader Context}

In this section, we survey research areas that are related to and overlap with TUIs. We also discuss literature that interprets TUIs as part of an emerging generation of HCI, or a larger research endeavor. We begin by describing the fields of Tangible Augmented Reality, Tangible Tabletop Interaction, Ambient displays, and Embodied Interaction. We then discuss unifying perspectives such as Tangible Computing, Tangible Interaction, and Reality-Based Interaction.

\subsection{Related Research Areas}

Various technological approaches in the area of next generation user interfaces have been influencing each other, resulting in mixed approaches that combine different ideas or interaction mechanisms. Some approaches, such as ambient displays, were originally conceived as part of the Tangible Bits vision, others can be considered a specialized type of TUI or as sharing characteristics with TUIs.

\subsubsection{Tangible Augmented Reality}

Tangible Augmented Reality (Tangible AR) interfaces [132, 148, 263] combine tangible input with an augmented reality display or output. 
The virtual objects are "attached" to physical objects that the user manipulates. A 3D-visualization of the virtual object is overlaid onto the physical manipulative which is tagged with a visual marker (detectable with computer vision). The digital imagery becomes visible through a display, often in the form of see-through glasses, a magic lens, or an augmented mirror. Such a display typically shows a video image where the digital imagery is inserted at the same location and $3 \mathrm{D}$ orientation as the visual marker. Examples of this approach include augmented books [18, 263] and tangible tiles [148].

\subsubsection{Tangible Tabletop Interaction}

Tangible tabletop interaction combines interaction techniques and technologies of interactive multi-touch surfaces and TUIs. Many tangible interfaces use a tabletop surface as base for interaction, embedding the tracking mechanism in the surface. With the advancement in interactive and multi-touch surfaces the terminology has become more specific, tabletop interaction referring predominantly to finger-touch or penbased interaction. But simultaneously, studies within the research area of interactive surfaces increasingly investigate mixed technologies [135], typically utilizing a few dedicated tangible input devices and artifacts on a multi-touch table. Research in this field is starting to investigate the differences between pure touch-based interaction and tangible handles (e.g., [232]) and to develop new techniques for optical object sensing through the surface (e.g., [118]). Toolkits such as reacTIVision [125] enable a blend of tangible input and multi-touch, the most prominent example being the reacTable [125], a tool for computer music performers.

\subsubsection{Ambient Displays}

Ambient displays were originally a part of Ishii's Tangible Bits vision [117], but soon developed into a research area of its own, many ambient displays being based on purely graphical representations on monitors and wall displays. The first example of an ambient display with a physical world realization is likely Jerimijenko's LiveWire. 
Greenberg and Fitchett [82] describe a range of student projects that used the Phidgets toolkit to build physical awareness devices, for example, a flower that blooms to convey the availability of a work colleague. The active-Hydra project [83] introduced a backchannel, where user's proximity to and handling of a figurine affect the fidelity of audio and video in a media window (an always-on teleconference). Some more recent projects employ tangible interfaces as ambient displays. Many support distributed groups in maintaining awareness [23], using physical artifacts for input as well as output. Commercial applications include the Nabaztag bunnies, which in response to digital events received via a network connection blink and move their ears. Edge and Blackwell [51] suggest that tangible objects can drift between focus and periphery of a user's attention and present an example of peripheral (and thus ambient) interaction with tangibles. Here tangible objects on a surface next to an office worker's workspace represent tasks and documents, supporting personal and group task management and coordination.

\subsubsection{Embodied User Interfaces}

The idea of embodied user interfaces [54, 64] acknowledges that computation is becoming embedded and embodied in physical devices and appliances. The manual interaction with a device can thus become an integral part of using an integrated physical-virtual device, using its body as part of the interface:

"So, why can't users manipulate devices in a variety of ways - squeeze, shake, flick, tilt - as an integral part of using them? (...) We want to take user interface design a step further by more tightly integrating the physical body of the device with the virtual contents inside and the graphical display of the content." [64]

While research prototypes have been developed since 2000, only with the iPhone has tilting a device become a standard interaction technique, the display changing orientation accordingly. While conceived of as an interface vision of its own, the direct embodiment of 

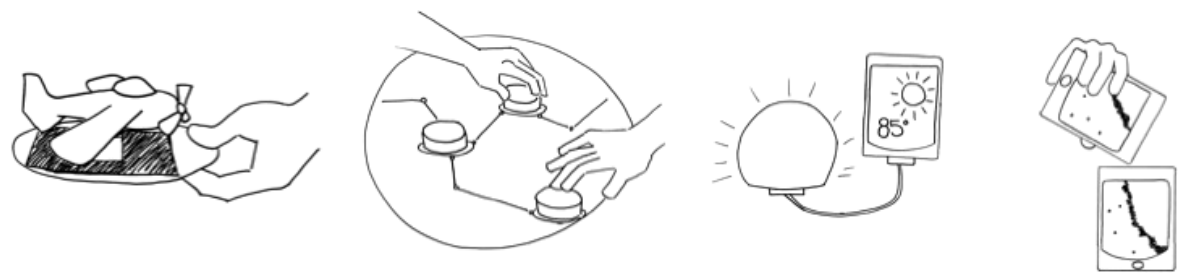

Fig. 3.1 Research areas related to TUIs. From left to right: Tangible Augmented Reality, virtual objects (e.g., airplane) are "attached" to physically manipulated objects (e.g., card); Tangible Tabletop Interaction, physical objects are manipulated upon a multi-touch surface; Ambient Displays, physical objects are used as ambient displays; Embodied User Interfaces, physical devices are integrated with their digital content.

computational functionality can be considered a specialized type of tangible interface where there is only one physical input object (which may have different parts that can be manipulated).

\subsection{Unifying Perspectives}

\subsubsection{Tangible Computing}

Dourish [50] discusses multiple concepts that are based on the idea of integrating computation into our everyday world under the term tangible computing. These concepts include TUIs, Ubiquitous Computing, Augmented Reality, Reactive Rooms, and Context-Aware Devices. Tangible Computing covers three trends: distributing computation over many specialized and networked devices in the environment, augmenting the everyday world computationally so that it is able to react to the user, and enabling users to interact by manipulating physical objects. The concepts share three characteristics [50]:

- no single locus of control or interaction. Instead of just one input device, there is a coordinated interplay of different devices and objects;

- no enforced sequentiality (order of actions) and no modal interaction; and

- the design of interface objects makes intentional use of affordances which guide the user in how to interact. 
Embedding computation in the environment creates embodied interaction - it is socially and physically situated. As a core research question Dourish [50] identifies the relation of actions with the space in which they are performed. This refers to the configuration of the environment effecting computational functionality, and the position and orientation of the user being relevant for how actions are interpreted (e.g., a device is activated if one walks toward it). The term tangible computing emphasizes the material manifestation of the interface (this is where tangible interfaces go the farthest) and the embedding of computing in the environment.

Tangible Interfaces differ from the other approaches by making evident that representations are artifacts in their own right that the user can directly act upon, lift up, rearrange, sort and manipulate [50]. In particular, at one moment in time, several levels of meaning can be present. Moving a prism token in Illuminating Light (a physics learning system that emulates a laser light installation with laser beams and prisms on a surface) [240] can be done simply to make space, to explore the system response, as moving the prism (seeing the token as stand-in), as moving the laser beam (using the token as a tool), or to manipulate the mathematical simulation underneath (the entire system is a tool). The user can freely switch attention between these different levels. This seamless nesting of levels is made possible through the embodiment of computation.

\subsubsection{Tangible Interaction}

Hornecker and Buur [105] suggest the term tangible interaction to describe a field of approaches related to, but broader than TUIs. They argue that many systems developed within arts and design aimed at creating rich physical interactions share characteristics with TUIs. But the definitions used to describe tangible user interfaces are too restrictive for these related areas. Instead of focusing on providing tangible "handles" (physical pointers) to support the manipulation of digital data, many of these related systems aim at controlling things in the real world (e.g., a heating controller) or at enabling rich or skilled bodily interaction [29]. In the latter case the emphasis lies more on the 
expressiveness and meaning of bodily movement and less on the physical device employed in generating this movement or the "data" being manipulated.

The tangible interface definition "using physical objects to represent and manipulate digital data" is identified as a data-centered view because this phrasing indicates that data is the starting point for design. The expressive-movement view, in contrast, focuses on bodily movement, rich expression and physical skill, and starts design by thinking about the interactions and actions involved. In the arts, a space-centered view is more dominant, emphasizing interactive and reactive spaces where computing and tangible elements are means to an end and the spectator's body movement can become an integral part of an art installation. Interaction designers have also developed an interest in bodily interaction, which can be pure movement (gestures, dance) or is related to physical objects.

Tangible Interaction adopts a terminology preferred by the design community, which focuses on the user experience and interaction with a system [14, 243]. As an encompassing perspective it emphasizes tangibility and materiality, physical embodiment of data, bodily interaction, and the embedding of systems in real spaces and contexts. This embeddedness is why tangible interaction is always situated in physical and social contexts (cf. [50]).

\subsection{Reality-Based Interaction}

Jacob et al. [119] proposed the notion of reality-based interaction as a unifying framework that ties together a large subset of emerging interaction styles and views them as a new generation of HCI. This notion encompasses a broad range of interaction styles including virtual reality, augmented reality, ubiquitous and pervasive computing, handheld interaction, and tangible interaction [119].

The term reality-based interaction results from the observation that many new interaction styles are designed to take advantage of users' well-entrenched skills and experience of interacting with the real nondigital world to a greater extent than before. That is, interaction with digital information becomes more like interaction with the real world. 

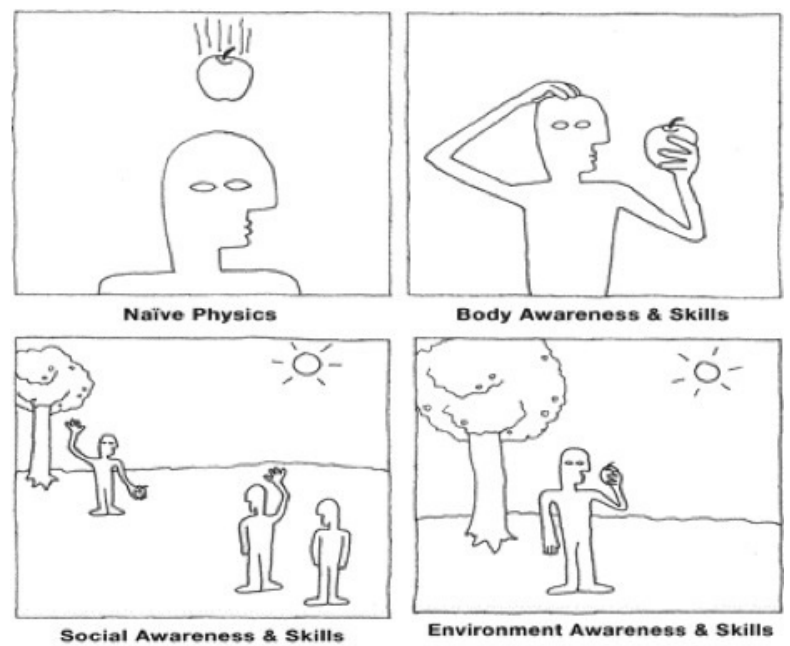

Fig. 3.2 Four themes of reality-based interaction [119].

Furthermore, emerging interaction styles transform interaction from a segregated activity that takes place at a desk to a fluid free form activity that takes place within the non-digital environment. Jacob et al. [119] identified four themes of interaction with the real world that are typically leveraged (see Figure 3.2):

- Naïve Physics: the common sense knowledge people have about the physical world.

- Body Awareness and Skills: the awareness people have of their own physical bodies and their skills of controlling and coordinating their bodies.

- Environment Awareness and Skills: the sense of surroundings people have for their environment and their skills of manipulating and navigating their environment.

- Social Awareness and Skills: the awareness people have that other people share their environment, their skills of interacting with each other verbally or non verbally, and their ability to work together to accomplish a common goal.

These four themes play a prominent role and provide a good characterization of key commonalities among emerging interaction styles. 
Jacob et al. further suggest that the trend toward increasing realitybased interaction is a positive one, because basing interaction on preexisting skills and knowledge from the non-digital world may reduce the mental effort required to operate a system. By drawing upon preexisting skills and knowledge, emerging interaction styles often reduce the gulf of execution [168], the gap between users' goals for actions and the means to execute those goals. Thus, Jacob et al. encourage interaction designers to design their interfaces so that they leverage reality-based skills and metaphors as much as possible and give up on reality only after explicit consideration and in return for other desired qualities such as expressive power, efficiency, versatility, ergonomics, accessibility, and practicality.

The reality-based interaction framework is primarily a descriptive one. Viewing tangible interfaces through this lens provides explanatory power. It enables TUI developers to analyze and compare alternative designs, bridge gaps between tangible interfaces and seemingly unrelated research areas, and apply lessons learned from the development of other interaction styles to tangible interfaces. It can also have a generative role by guiding researchers in creating new designs that leverage users' pre-existing skills and knowledge. To date, most TUIs rely mainly on users' understanding of naïve physics, simple body awareness, and skills such as grasping and manipulating physical objects as well as basic social skills such as the sharing of physical objects and the visibility of users' actions. The RBI frameworks highlights new directions for TUI research such as the use of a much richer vocabulary of body awareness and skills as well as the leveraging of environment awareness skills. 


\section{Application Domains}

In this section we discuss a sample of existing TUIs. While some of the interfaces we discuss here are central examples that are obviously considered a TUI, others are more peripheral and have TUI-like characteristics. The goal of the paper is to describe these characteristics and provide readers with ways for thinking and discussing them rather than bounding what a TUI is or is not. Dominant application areas for TUIs seem to be learning, support of planning and problem solving, programming and simulation tools, support of information visualization and exploration, entertainment, play, performance and music, and also social communication. Recently, we have seen an even wider expansion of application examples into areas such as facilitating discussions about health information among women in rural India [179], tracking and managing office work [51], or invoice verification and posting [112].

The domains we discuss here are not mutually exclusive, as very often a TUI can be, for example, a playful learning tool. For some areas there are already specialized accounts. An excellent and detailed overview of the argumentations for learning with tangibles and of the research literature available in 2004 is provided in a Futurelab report on Tangibles and Learning [174]. Jorda [124] provides an overview of the history of and motivation for music performance tangible interfaces. 


\subsection{TUls for Learning}

A large number of TUIs can be classified as computer-supported learning tools or environments. There are several underlying reasons for this. First, learning researchers and toy designers have always followed the strategy of augmenting toys to increase their functionality and attractiveness. Second, physical learning environments engage all senses and thereby support the overall development of the child. With reference to Bruner and Piaget, research and theory on learning stresses the role of embodiment, physical movement, and multimodal interaction (cf. $[6,174])$. Furthermore, studies on gesture have shown how gesturing supports thinking and learning [80]. Moreover, if a system supports learning the fundamentals of a particular domain and is thus aimed at beginners, it rarely needs to cater for complex or large examples. TUI developers thus evade some of the design problems inherent for TUIs (see Section 9 on strength and limitations of TUIs), such as scaling up to large numbers of objects or connections between objects and of screen estate or "physical space". A TUI might also abstract from some of the details that beginners do not deal with yet.

A range of learning systems relates to the categories of problem solving, planning, and simulation systems, which are described in detail later-on. These include, for example, Tinkersheets, which supports learning about logistics [267], and Illuminating Light [240], a learning environment for holography and optics. Many TUI systems also combine learning with entertainment, as is the case for educational toys or museum installations. We here mention some learning-related systems that also belong to other categories, but defer the discussion of TUIs for tangible programming to a separate section.

Digital Manipulatives [199, 266] are TUIs that build on educational toys such as construction kits, building blocks, and Montessori materials. They are computationally enhanced versions of physical objects that allow children to explore concepts, which involve temporal processes and computation. Well known and commercially marketed as Lego Mindstorms ${ }^{\mathrm{TM}}$ is the Lego/Logo robotic construction kit that evolved from the MIT Media Lab Lifelong Kindergarten group [198]. A newer addition to this family line are the Pico crickets, which enable 


\section{Application Domains}

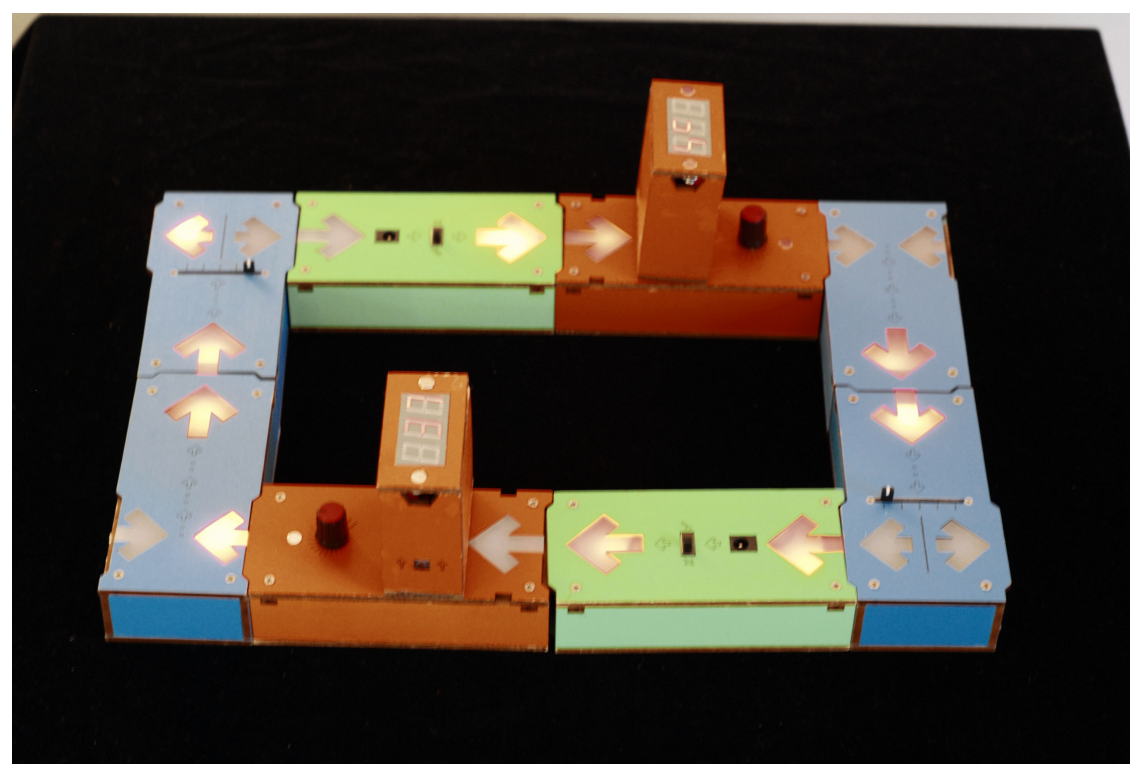

Fig. 4.1 The Flow Blocks [266] allow children to explore concepts relevant for understanding causality. The blocks can be annotated to represent a real-world system such as virus spread in a population. The blocks light up to show the data flow, and children can probe the current values being propagated through a block by sticking a little display onto it (image courtesy of Oren Zuckerman).

children to build their own apparatus for scientific experiments from sensors, actuators, and robotic parts (http://www.picocricket.com/).

Computationally enhanced construction kits can make concepts accessible on a practical level that are normally considered to be beyond the learner's abilities and age-related level of abstract thinking. Smart Blocks is an augmented mathematical manipulative that allows learners to explore the concepts of volume and surface area of $3 \mathrm{D}$ objects constructed by the user [79]. Schwiekard et al. [212] investigate how a tangible construction kit can be used to explore graph theory. A range of digital manipulatives support exploration of movement. For example, Curlybot [73] is a robotic ball that records its movement on a surface and then replays this movement repeatedly. Topobo [196] enables the building of robotic creatures from parts (see Figure 4.2), where movement of special joints can be programmed individually through demonstration. Similar joints can also copy the movement demonstrated on 

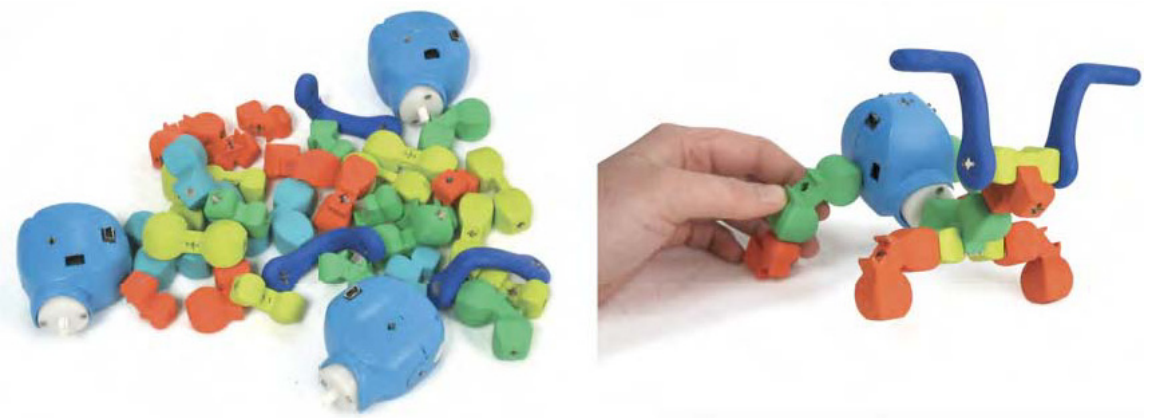

Fig. 4.2 Topobo [196] consists of connectible movable parts that are attached to an "active" joint (the slightly bigger, blue part) that can record and replay motion of attached parts (images from http://www.topobo.com/topobo-hires/index.html courtesy: Hayes Raffle).

one joint. As a learning tool, Topobo enables children to learn about balance, movement dynamics, and anatomy.

A compelling application for TUIs seems to be storytelling, supporting early literacy education. Storytelling applications build on traditional toys and play environments or books, and augment these. Ryokai and Cassell [204] developed Storymat, a play carpet that can record and replay children's stories. Storymat detects RFID-tagged toys that are placed upon it. In replay, an image of the moving toy is projected onto the carpet and the recorded audio played. The Kidstory project [173] tagged children's drawings so children could interact and navigate physically with wall projections. A range of projects have followed on from these early endeavors, often combining augmented reality techniques with tangible interface notions (e.g. [263]). Africano et al. [2] present "Ely the Explorer", an interactive play system that supports collaboraive learning about geography and culture while practicing basic literacy skills. The system mixes touch-screen technology, use of physical knobs to interact with screen content, tangible toys, and RFID-tagged cards. Related to literacy education is WebKit, a system supporting the teaching of rhetorical skills to school children [227]. Using tagged statement cards, children can prepare an argument, order and connect them with supporting evidence (i.e., webpages) by placing cards on a row of argument squares. Then, children "walk" a special speaker tag across the argument squares from first to last as they deliver their speech. 
A more recent development is TUI's supporting learning for children with special needs. Digital construction kits such as Topobo [196] and Lego Mindstorms ${ }^{\mathrm{TM}}$ are increasingly used within educational robotics specifically for special needs education [248]. Hengeveld has systematically explored the design space for speech therapy through storyreading for severely handicapped toddlers in the Linguabytes project [93, 94], see Figure 4.3. Physical interaction here has benefits of slowing down interaction, training perceptual-motor skills, providing sensorial experience, supporting collaborative use, and giving more control to the toddler. Overall, a tangible interface can provide access to a rich learning environment with more opportunities for cognitive, linguistic, and social learning than a traditional GUI system.

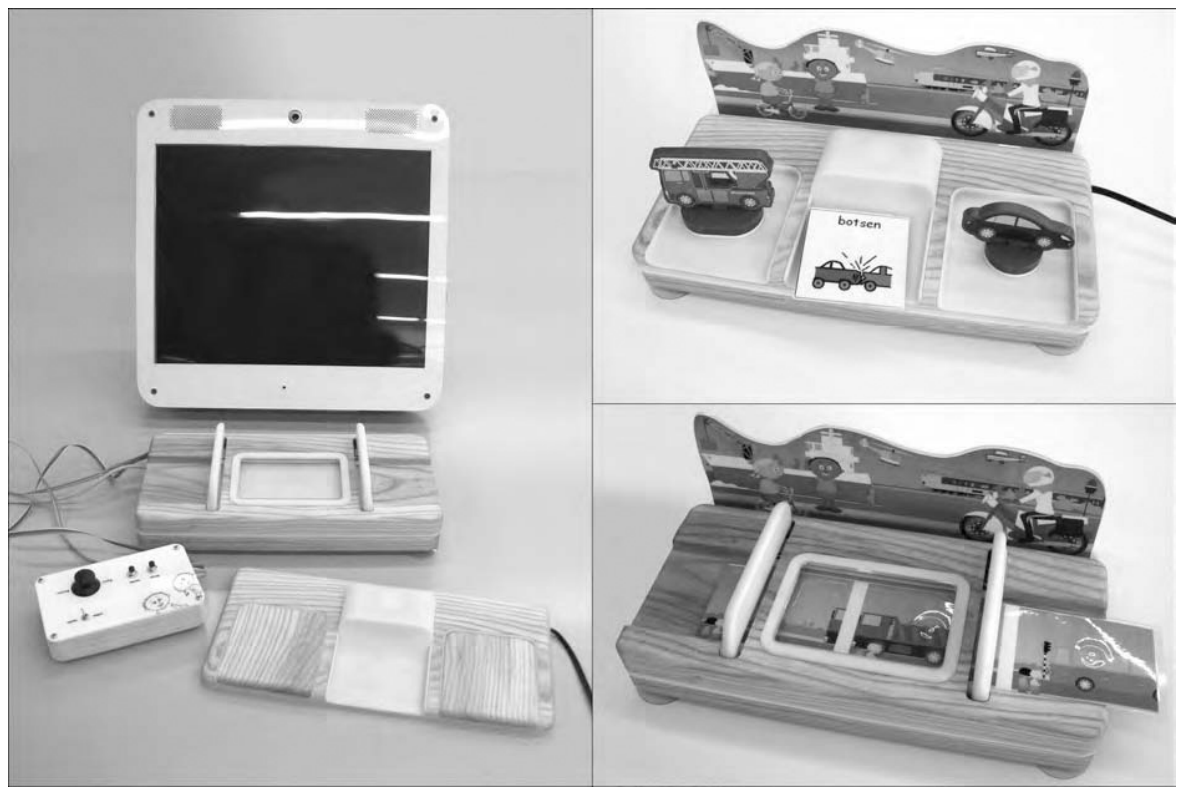

Fig. 4.3 Linguabytes [94], a TUI for improving speech-therapy sessions with severely impaired children. Left: the overall system, including the control unit for the therapist and a monitor that displays animated sequences in response to the child's actions. Right, Bottom: a storybook is sled into the top rim of the board, activating a subunit, here on traffic. The booklet can be moved back and forth. Placed behind the unit, a corresponding scene is triggered as an animation with audio on a screen. Top: The trays recognize the wooden images placed onto them. In this unit the objects are combined into sentences to train syntax (photo: Bart Hengeveld). 
A few TUIs have also been developed for diagnostic purposes, exploiting the capability of the digitized structure to log manipulation. The kinds of mistakes and steps taken in building a spatial structure after a given model can indicate the level of cognitive spatial abilities a child has developed or the effects of a brain injury on adults [218]. Other projects develop toys that record interaction, providing data to assess a child's manual and cognitive development [256].

\subsection{Problem Solving and Planning}

Three aspects of TUIs have been demonstrated as effective in supporting problem solving: (1) epistemic actions, (2) physical constraints, and (3) tangible representations of a problem. Epistemic actions [137] are the non-pragmatic manipulations of artifacts aimed at better understanding a task's context. Such actions have been shown to facilitate mental work [137]. TUIs support a wide range of epistemic actions ranging from rotating physical objects in space to arranging them upon a surface. Physical constraints can make use of physical affordance to communicate interaction syntax and to limit the solution space. Thus, physical constraints can decrease the need for learning explicit rules and lower the threshold for using a computational system for a particular task [239]. Finally, tangible representation is most compelling in spatial or geometric application domains such as urban planning and architecture where the physical arrangement and manipulation of objects has a direct mapping to the represented problem. It has been found that using a TUI can support designers' spatial cognition, reduce cognitive load, and enable more creative immersion in the problem [134]. However, several studies have also demonstrated the benefits of tangible interaction with abstract information tasks [120, 180].

Following, we describe several TUI instances aimed at problem solving, planning, and simulation. We first review TUIs that represent domains with direct physical mapping. Then, we review TUIs for abstract information tasks.

Urp [241] (see Figure 2.1), is a TUI for urban planning that allows users to collaboratively manipulate a series of physical building models and tools upon a surface, in order to perform an analysis of shadows, 
proximities, reflections, wind, and visual space. While users place and manipulate building models upon the surface, the interface overlays digital information onto the surface, activating and updating multiple simulations. In addition to physical building models, Urp also provides a collection of physical tools for manipulating environmental conditions such as time of day and wind direction. By allowing users to collaboratively interact with physical objects, Urp provides an intuitive way to interact with complex computational simulations.

Similar to Urp, MouseHaus Table [108] enables urban planners to intuitively and collaboratively interact with a pedestrian simulation program by placing and manipulating everyday objects upon a surface. The ColorTable [154] supports urban planners and diverse stakeholders in envisioning urban change by providing them with means for coconstructing mixed-reality scenes against a background. The interface supports users in collaboratively building, animating, and changing a scene. SandScape and Illuminating Clay [115] are TUIs for designing and understanding landscapes (Figure 4.4). The users can alter the

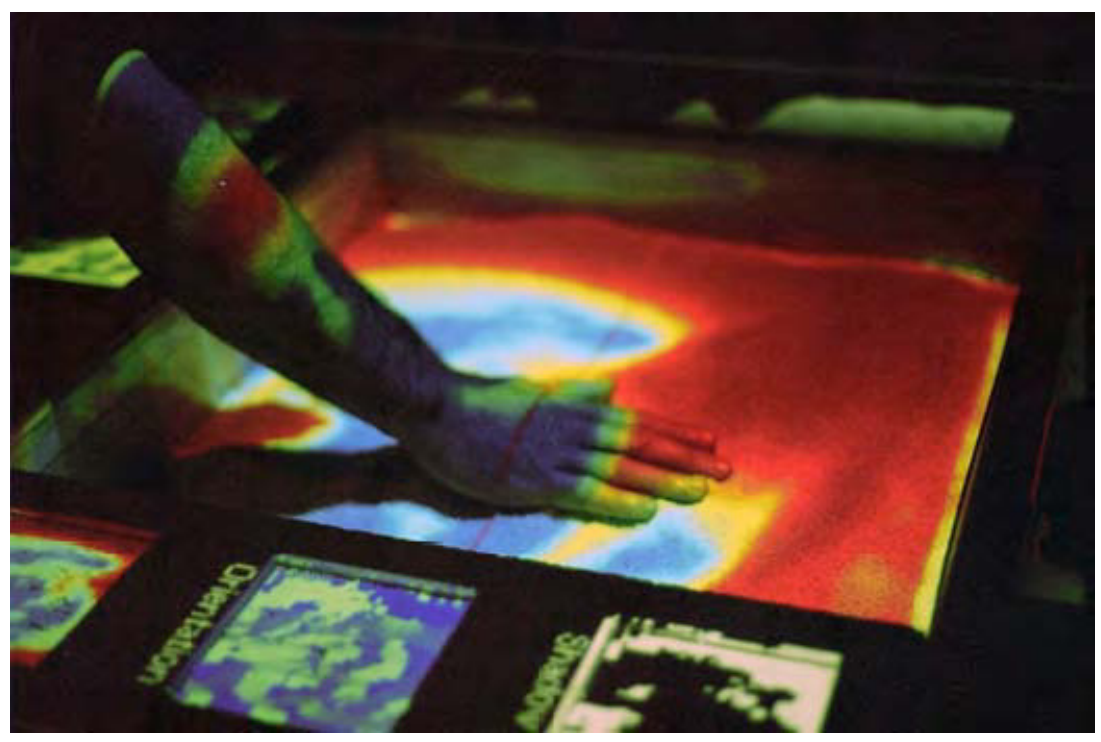

Fig. 4.4 The Sandscape [115] system as exhibited at the Ars Electronica Museum in Linz. The color projected onto the surface depicts the height profile. Putting a wooden block on the mode selection menu bar visible on the lower end of the image changes the visualization to indicate, e.g., erosion (photo: E. Hornecker). 


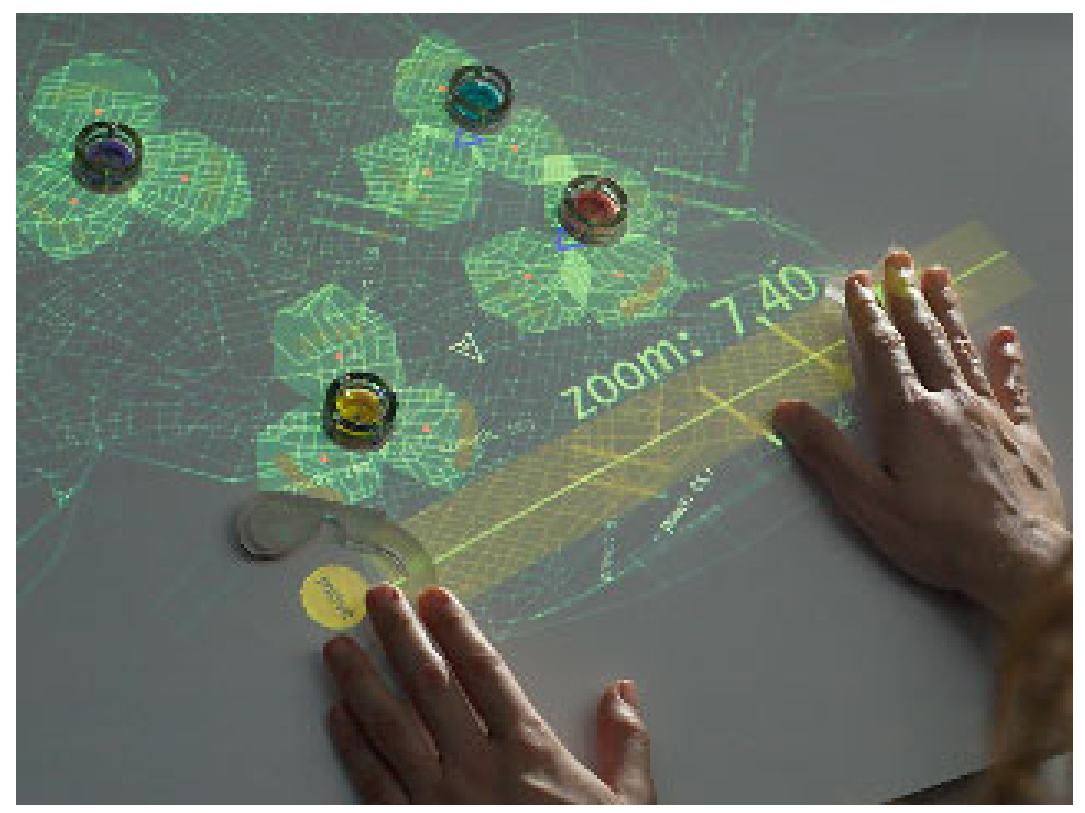

Fig. 4.5 Pico [181] is an interactive surface that supports solving complex spatial layout problems through improvization, Pico allows users to employ everyday physical objects as interface elements that serve as mechanical constraints within the problem space (Photo courtesy: James Patten).

form of a landscape model by manipulating sand or clay while seeing the resultant effects of computational analysis generated and projected on the landscape in real time.

Physical Intervention in Computational Optimization (Pico) [181], is a TUI based on a tabletop surface that can track and move small objects on top of it. The position of these physical objects represents and controls application variables (see Figure 4.3). The Pico interface has been used to control an application for optimizing the configuration of cellular telephone network radio towers. While the computer autonomously attempts to optimize the network, moving the objects on the table, the user can constrain their motion with his or her hands, or using other kinds of physical objects (e.g., rubber bands). A comparative study of Pico demonstrated that subjects were more effective at solving a complex spatial layout problem using the Pico system than with either of two alternative interfaces that did not feature actuation. 
Beyond architecture and urban planning, several TUI instances were developed to support problem solving and simulation in application domains of topological nature. Examples include Illuminating Light [240], a learning environment for holography and optics where plastic objects replace the real (and expensive) elements. Light beams are projected onto the setup from above, simulating light beams emitted from a light source and diverted by mirrors and prisms. In addition, angles, distances, and path length are projected into the simulation. Another example is an interactive surface for collaborative IP network design [141], which supports collaborative network design and simulation by a group of experts and customers. Using this system, users can directly manipulate network topologies, control parameters of nodes and links using physical pucks on the interaction table, and simultaneously see the simulation results projected onto the table in real time. Additional examples include a hub and strut TUI for exploring graph theory [212] and a constructive assembly for learning about system dynamics [266].

Only few examples exist of TUIs that explore the use of tangible interaction within a wider range of abstract information tasks. Tinkersheets [267] is a simulation environment for warehouse logistics used in vocational education (see Figure 4.6). It combines tangible models of shelving with paper forms, where the user can set parameters of the simulation by placing small magnets on the form. Edge and Blackwell [51] present a system that supports planning and keeping track of office work where tangible tokens on a special surface represent major work documents and tasks. Projections around a token visualize the progress and state of work, and through nudging and twisting tokens the user can explore their status and devise alternative plans, e.g., for task end dates. Finally, Senseboard [120] is a TUI for organizing and grouping discrete pieces of abstract information by manipulating pucks within a grid. An application for scheduling conference papers using the Senseboard was developed and evaluated. Its evaluation provides evidence that Senseboard is a more effective means of organizing, grouping, and manipulating data than either physical operations or graphical computer interaction alone. 


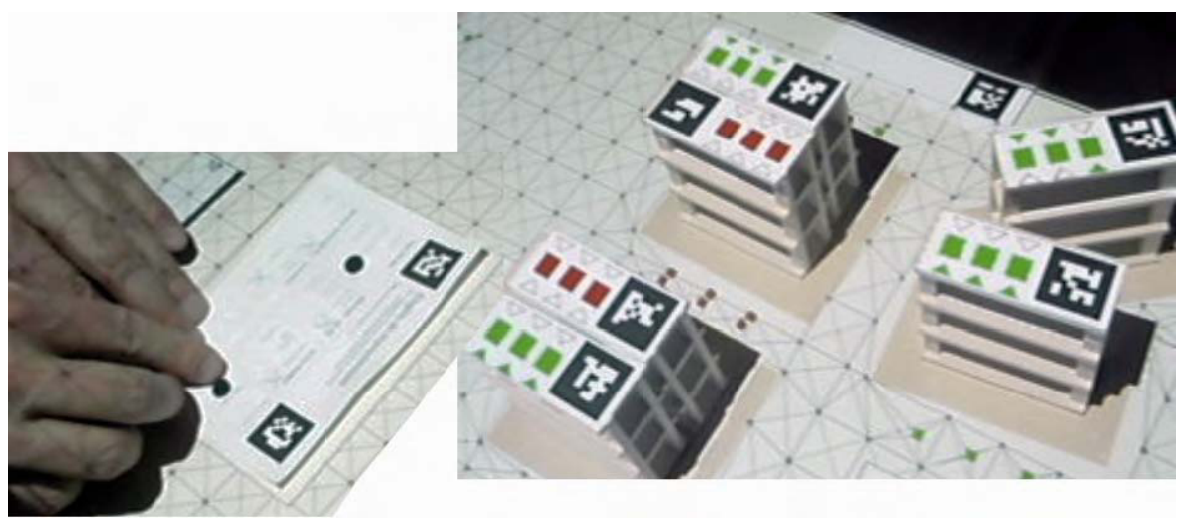

Fig. 4.6 Tinkersheets [267] supports learning about warehouse logistics and enables users to set simulation parameters through interaction with paper forms where small black magnets are placed onto parameter slots (photo: E. Hornecker).

\subsection{Information Visualization}

By offering rich multimodal representation and allowing for two-handed input, tangible user interfaces hold a potential for enhancing the interaction with visualizations. Several systems illustrate the use of tangible interaction techniques for exploring and manipulating information visualizations. Following we describe some example TUIs. We focus on TUIs that were fully implemented and evaluated with users.

The Props-Based Interface for 3D Neurosurgical Visualization [95] is a TUI for neurosurgical visualization that supports two-handed physical manipulation of handheld tools in free space. The tangible representation of the manipulated data consists of a doll-head viewing prop, a cutting-plane prop, and a stylus prop that help a surgeon to easily control the position and the angle of a slice to visualize by simply holding a plastic plate up to the doll head to demonstrate the desired cross-section. The system was informally evaluated with over fifty neurosurgeons. This evaluation has shown that with a cursory introduction, surgeons could understand and use the interface within about one minute of touching the props. GeoTUI [42] is a TUI for geophysicists that provides physical props for the definition of cutting planes on a geographical map that is projected upon a surface. The system enables geophysicists to select a cutting plane by manipulating a ruler prop 
or selection handles upon the projected map. The system was evaluated with geophysicists at their work place. The evaluation showed that users of the tangible user interface performed better than users of a standard GUI for a cutting line selection task on a geographical subsoil map.

Ullmer et al. [239] developed two tangible query interface prototypes that use physical tokens to represent database parameters (Figure 4.7). These tokens are manipulated upon physical constraints such as tracks and slots, which map compositions of tokens onto interpretations including database queries, views, and Boolean operations. This approach was evaluated and shown to be a feasible approach for constructing simple database queries, however, the evaluation did not show a performance advantage over a traditional GUI interface. Gillet

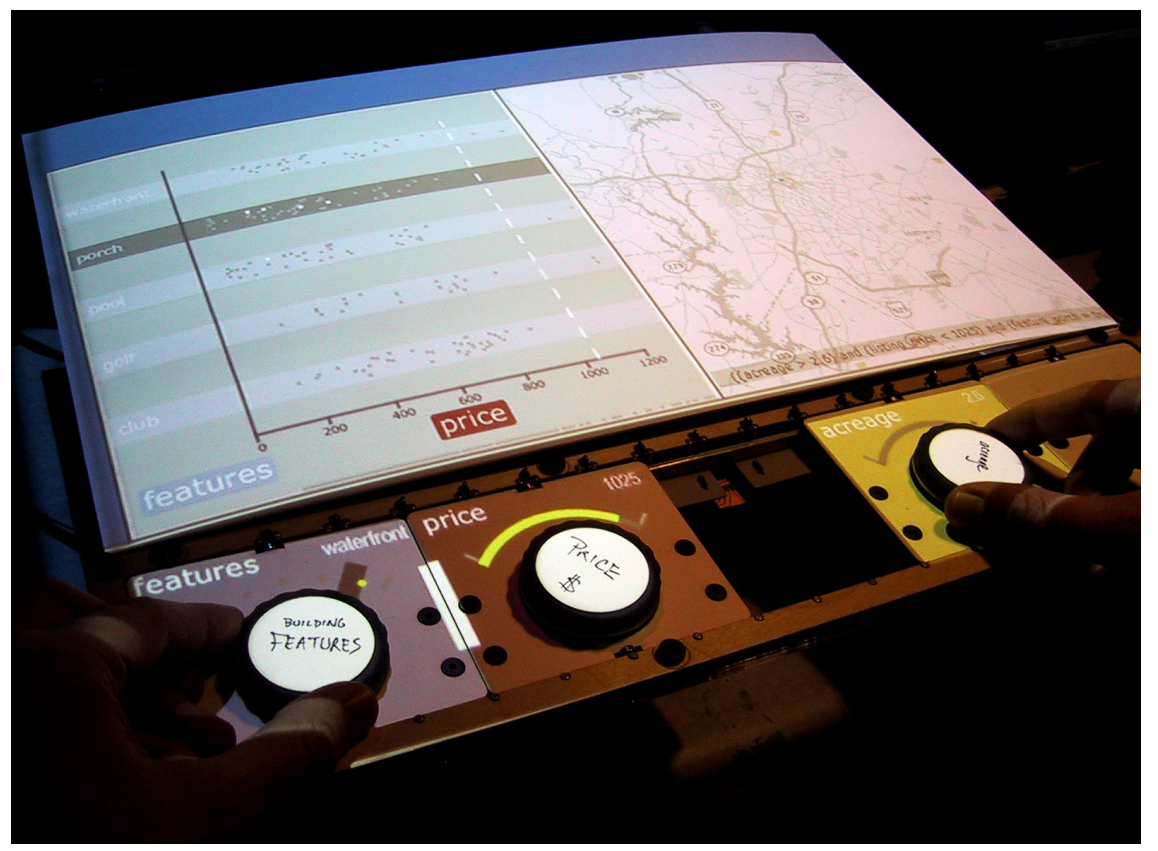

Fig. 4.7 Tangible Query Interfaces [239], a TUI for querying relational databases. The wheels represent query parameters. When placed within the query rack the distance between the wheels determines the logical relations between the query parameters. In the picture, the user constructs a query with three parameters, an AND operator is applied to the two wheels (parameters) on the left that are in close proximity, an OR operator is applied to the third wheel (parameter) on the right (Photo: courtesy of Brygg Ullmer). 
et al. [78] present a tangible user interface for structural molecular biology. It augments a current molecular viewer by allowing users to interact with tangible models of molecules to manipulate virtual representations (such as electrostatic field) that are overlaid upon the tangible models. Preliminary user studies show that this system provides several advantages over current molecular viewer applications. However, to fully understand the benefits of this approach the system requires further evaluation.

\subsection{Tangible Programming}

The concept of tangible programming, the use of tangible interaction techniques for constructing computer programs, has been around for almost three decades since Radia Perlman's Slot Machine interface [185] was developed to allow young children to create physical Logo programs. Suzuki and Kato coined the term Tangible Programming in 1993 to describe their AlgoBlocks system [230]. McNerney [161] provides an excellent historical overview of electronic toys developed mainly at MIT that are aimed at helping children to develop advanced problem-solving skills. Edge and Blackwell [52] present a set of correlates of the Cognitive Dimensions (CDs) of Notations frameworks specialized for TUIs and apply this framework to tangible programming systems. Their CDs analysis provides means for considering how the physical properties of tangible programming languages influence the manipulability of the information structure created by a particular language. Following, we discuss a number of TUIs that have presented techniques for programming, mainly in the context of teaching abstract concepts in elementary education.

AlgoBlocks [230, 231] supports children in learning programming, using a video-game activity. Big blocks represent constructs of the educational programming language Logo. These can be attached to each other forming an executable program, with the aim to direct a submarine through an underwater maze. During execution, an LED on each block lights up at the time the command is executed. The size of the blocks and physical movement of manipulating was argued to improve coordination and awareness in collaborative learning. 


\section{Application Domains}

Several TUIs allow children to teach an electronic toy to move by repeating a set of guiding motions or gestures. Examples include Topobo [196], Curlybot [73], and StoryKits [223]. This approach for programming is often referred to as programming by demonstration [43] or, as suggested by Laurel, programming by rehearsal [146]. Other systems support the construction of physical algorithmic structures for controlling on-screen virtual objects (e.g., AlgoBlocks [230]), or physical lego structures and robots (e.g., Digital Construction Sets [161], Electronic Blocks [258], and Tern [103]). Such systems could be classified as constructive assemblies [239], systems in which users connect modular pieces to create a structure.

Many tangible programming systems use physical constraints to form a physical syntax that adheres to the syntax of a programming language. For example, Tern [103] (see Figure 4.8), consists of a collection of blocks shaped like jigsaw puzzle pieces, where each piece represents either a command (e.g., repeat) or a variable (e.g., 2). The physical form of Tern's pieces determines what type of blocks (command or variables) and how many blocks can be connected to each piece. Fernaeus and Tholander [57] developed a distinct approach to tangible programming that enables children to program their own simulation games while sitting on a floor mat in front of a projection. Instead of representing an entire program through tangible artifacts, an RFID reader has to be placed onto the mat (which spatially corresponds to the grid) and any new programming cards, representing objects or behaviors, are then placed on the reader. This approach can be characterized by a loose and only temporary coupling between

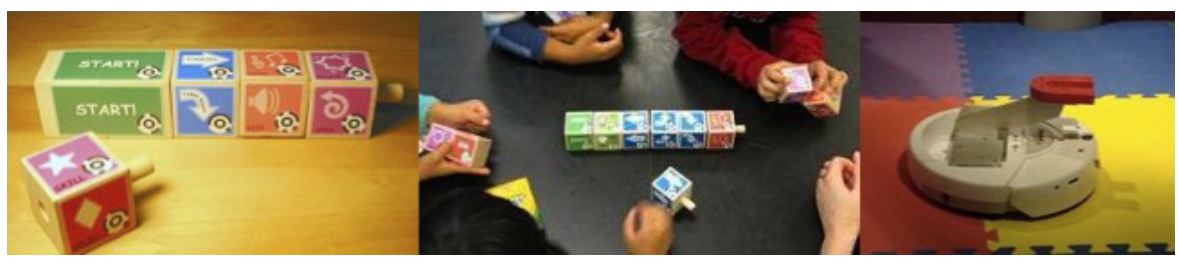

Fig. 4.8 Tern [103] is a tangible computer language designed for children in educational settings. Tern is featured in a permanent exhibit at the Boston Museum of Science called Robot Park. From left to right: Tern's blocks, collaborative programming using Tern, the programmable robot at the Robot Park exhibition (Photos: courtesy of Michael Horn). 
the physical and the screen, but was found to allow for more complex programs to be developed.

It is important to note that many tangible programming systems were designed to teach through free play and exploration and are hence perceived to hold an entertainment value. This perception may be one of the contributing factors for the wide acceptance and popularity of the tangible programming approach. However, until lately only little evidence has been provided that tangible programming offers educational benefits beyond those provided by visual programming languages. In a recent study Horn et al. compared the use of a tangible and a graphical interface as part of an interactive computer programming exhibit in the Boston Museum of Science [102]. The collected observations from 260 museum visitors and interviews with thirteen family groups provide evidence that children are more likely to approach, and are more actively engaged in a tangible programming exhibit. The effect seems to be especially strong for girls. This evidence shows that carefully designed tangible programming systems can indeed offer concrete educational benefits.

With relatively few exceptions, research on Tangible Programming so far has mostly focused on applications related to learning and play. Tangible query interfaces such as the aforementioned system by Ullmer et al. [239] can also be interpreted as a form of tangible programming. Another example in this area are the Navigational Blocks by Camarata et al. [32], built to support visitors at a multimedia information kiosk in Seattle's Pioneer Square district. Visitors can explore the history of the area by placing and rotating wooden blocks on a query table in front of a display monitor. Each block represents a category of information (e.g., who, when, events), and the sides represent different instances of a category (e.g., founding fathers, women, native Americans). The blocks are equipped with orientation sensors and electromagnets. Depending on whether two information category instances will yield information (e.g., an event involving native Americans) the blocks will attract or repel each other, providing actuated feedback. The notion of control cubes has been popular in research particularly for end-user programming, where the faces of a cube usually serve to program, e.g., home automation or as a remote control for consumer 
electronics [21, 28, 62]. Tangible programming by demonstration was utilized to program event-based simulations of material flow on conveyor belts in plants [208]. Users can define simple rules (e.g., "if machine A is occupied and machine B is free then route pallets to B") by placing tags on a physical model and moving tokens. This generates a Petri Net, which is finally transformed into SPSS code. Researchers at the Mads Clausen Institute in Denmark investigate the use of tangible interfaces in the context of industrial work in plants, in particular for supporting configuration work by service technicians [225]. This research attempts to bring back some of the advantages of traditional mechanical interfaces, such as exploitation of motor memory and physical skills, situatedness, and visibility of action.

\subsection{Entertainment, Play, and Edutainment}

TUI-related toys, entertainment and edutainment TUIs are overlapping application areas. The Nintendo Wii is probably the best example for a tangible device in entertainment, and its commercial success demonstrates the market potential of TUI-related systems. But we should not overlook other examples that more closely fit the TUI definition. Many modern educational toys employ the principles of physical input, tangible representation, and digital augmentation. For example, Neurosmith markets the MusicBlocks, which allow children to create musical scores by inserting colored blocks into the toy body, varying and combining the basic elements, and the SonicTiles, which allow children to play with the alphabet.

Many museum interactives that combine hands-on interaction with digital displays can be interpreted as TUIs. For example, at the Waltz dice game in the Vienna Haus der Music (Museum of Sound) visitors roll with two dice to select melodic lines for violin and recorder, from which a short waltz is automatically generated. The museum also hosts Todd Machover's Brain Opera installation, a room full of objects that generate sound in response to visitors' movement, touch and voice. An exhibition about DNA at the Glasgow Science Museum includes several exhibits that allow visitors to tangibly manipulate DNA strands to understand how different selections effect genes (Figure 4.9). In the 


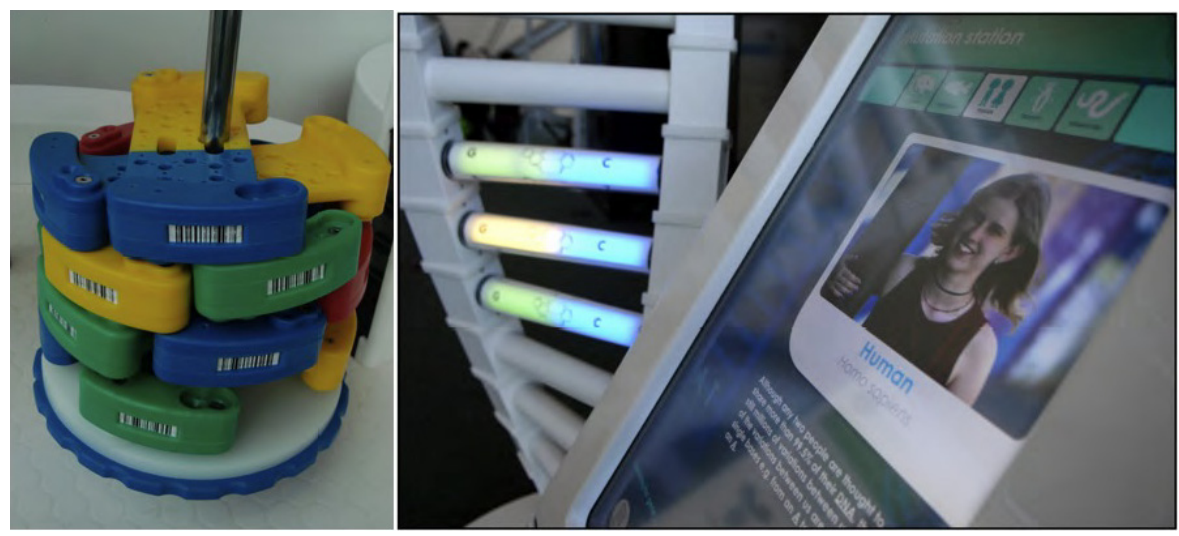

Fig. 4.9 The Inside DNA exhibition at the Glasgow Science Centre. Left: visitors create a DNA strand by stacking colored blocks with barcodes onto a shaft, and the strand is then read by rotating the tower past a reader. Right: at another exhibit, rotating the lighted tubes selects DNA. An attached screen then explains which kind of animal has been created (images courtesy: Glasgow Science Centre, 2009).

Children's museum in Boston placing two printed blocks in a recess to form the picture of a musical instrument triggers its sound.

Augmenting toys and playful interaction has for long been a focus in TUI research. We already covered many examples of playful learning TUIs for storytelling and building robotic creatures. A straightforward application of the tangible interface idea is to augment traditional board games, as does the Philips EnterTaible project [244]. These preserve the social atmosphere of board games while enabling new gaming experiences. Magerkurth et al. [152] introduce the STARS platform, which integrates personal mobile devices with a game table and tangible playing pieces. Players can access and manage private information on a PDA, serving as a secondary, silent information channel. The game engine alleviates users from tedious tasks such as counting money or setting up the board and can furthermore dynamically alter the board in response to the game action. Leitner et al. [149] present a truly mixed reality gaming table that combines real and virtual game pieces. Real objects are tracked by a depth camera and can become obstacles or a ramp in a virtual car race, or real and virtual dominos are connected to tumble into each other. 
Various systems attempt to bring digital activities such as sound and video capture back into the physical world. Labrune and Mackay [144] present Tangicam, a tangible camera that lets children capture and edit pictures and videos. Tangicam is shaped as a big circular handle with two embedded cameras. Holding the camera up frames the picture, and pressing the handle starts recording. Placed on an interactive table, the handle frame becomes a display space and can be used to slide through the video. Zigelbaum et al. [264] introduce the Tangible Video Editor, a tangible interface for editing digital video clips (see Figure 4.10). Clips are represented by physical tokens and can be manipulated by picking them up, arranging them in the work area (e.g., sorting and sequencing them), or attaching transition tokens between clips. IOBrush [205] is a digital drawing tool for young children to explore color, texture, and movement. Using a paintbrush that contains a video camera, they can "pick up" moving images, and then draw on a dedicated canvas (Figure 4.11). Jabberstamp [195] enables children to create drawings with embedded audio recordings using a rubber stamp in combination with a microphone to record and a toy trumpet as playback tool.

Hinske et al. [97] present guidelines for the design of augmented toy environments, and present an augmented version of a Playmobile Knights' Castle where movement of the figures triggers audio output (e.g., smoke). Interactive playgrounds can be seen as large-scale TUIs that users move around in. The Cardboard Box Garden [61] is an

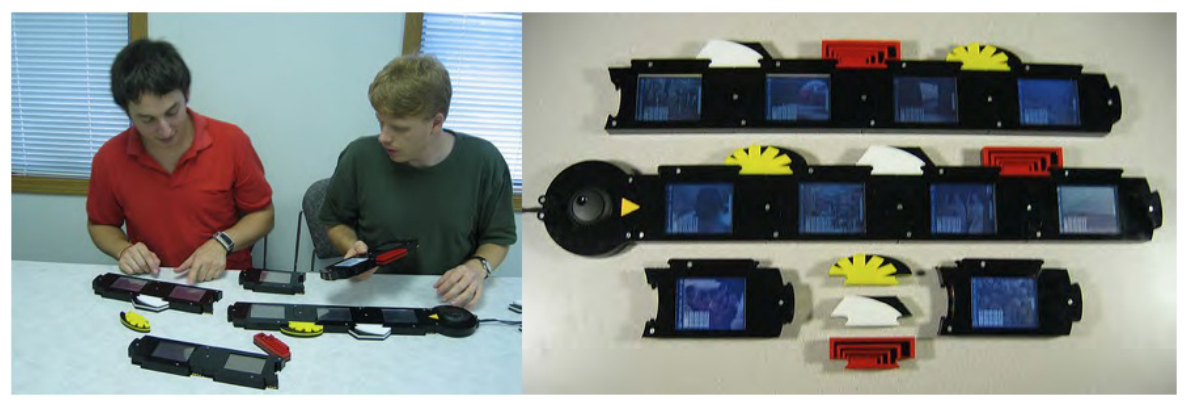

Fig. 4.10 The Tangible Video Editor [264], a TUI for editing digital video clips. Clips are represented by physical tokens shaped as jigsaw puzzle pieces, transitions are represented using colorful tokens that fit between connected clips (Photos courtesy of Jamie Zigelbaum). 


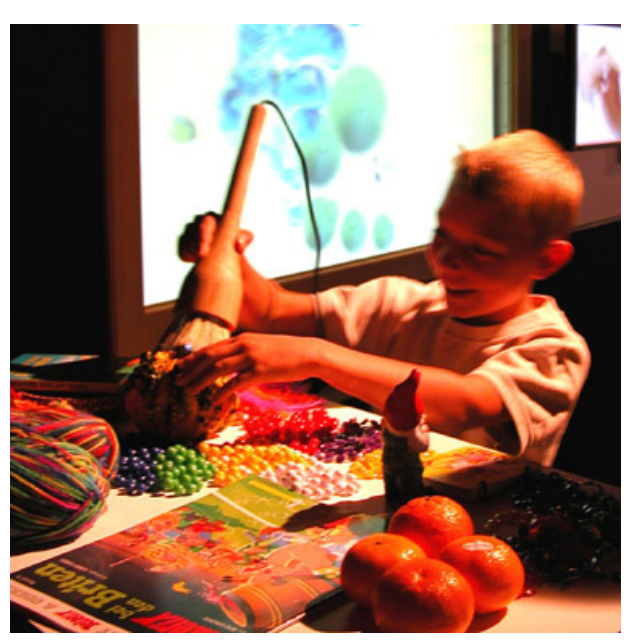

Fig. 4.11 I/O Brush [205] is a physical drawing tool to explore colors, textures, and movements found in everyday materials by allowing users to "pick up" images and draw with them (photo courtesy of Kimiko Ryokai).

interactive musical play environment for children built from large cardboard boxes. Some boxes start a sound recording whenever opened, others replay one of these tracks, and others control sound parameters. Stacking and moving boxes effects the overall sound arrangement. Sturm et al. [229] propose a research agenda for interactive playgrounds that react to children's interactions and actively encourage play. As key design issues they identify support of social interaction, simplicity combined with adequate challenge, goals (either open-ended or explicit) and motivating system feedback.

\subsection{Music and Performance}

Music applications are one of the oldest and most popular areas for TUIs, becoming ubiquitous around the millennium with projects such as Audiopad [182], BlockJam [167], the Squeezables [250], or the art installation SmallFish [74]. Jordà [124] identifies several properties of TUIs and multi-touch tables that make them a promising approach for music performance: support of collaboration and sharing of control; continuous, real-time interaction with multidimensional data; and support of complex, skilled, expressive, and explorative 
interaction. An overview of example systems is provided on Martin Kaltenbrunner's website [129]. Generally, we can distinguish between four high-level approaches for TUI music applications: instruments such as the reacTable [125], that are fully controllable sound generators or synthesizers, sequencer TUIs that mix and play audio samples, sound toys with limited user control, and controllers that remotely control an arbitrary synthesizer.

Music TUIs are either designed for the novice where they provide an intuitive and easily accessible toy, or aim at the professional that appreciates physical expressiveness, legibility, and visibility when performing electronic music in front of an audience. Besides of being an intriguing application area for research projects, many music TUIs are developed by professionals, such as electronic music artists. The NIME conference series (New Instruments for Musical Expression) is the most important conference venue in this area. The development of new hybrid instruments that make use of physical input and seemingly old-fashioned materials to enable users to experiment with sound has been pursued for several decades by renowned research and artist groups, such as the HyperInstruments group at MIT [250], or STEIM, an independent interdisciplinary studio for performance art in Amsterdam.

Tangible music interfaces have become visible at the forefront of TUI research through the reacTable [125] (Figure 4.12), a tabletop system that has given a new interface to modular synthesis programming

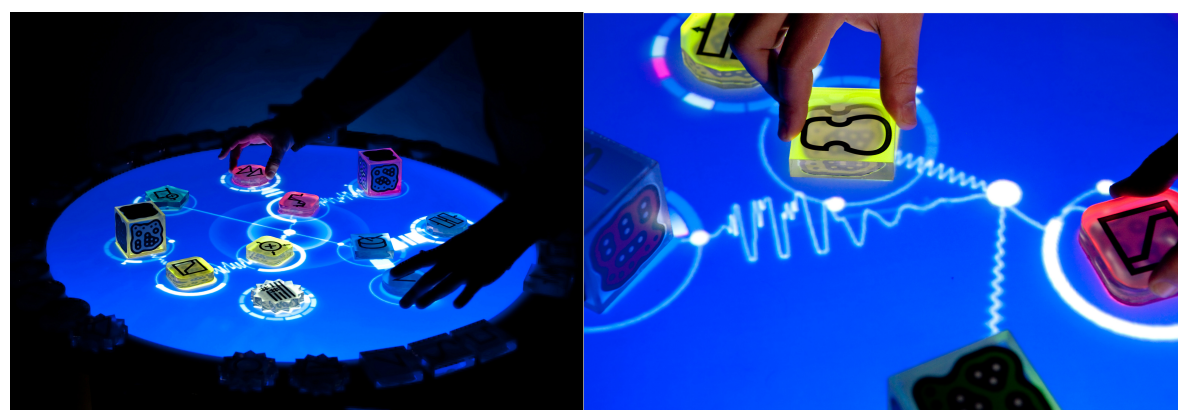

Fig. 4.12 The reacTable [125]. The parameters of the function that a physical token represents can be changed by touching the outline projected around the token. Bringing a new element into proximity of others results in "dynamic patching", the connections re-arranging themselves (Photos: Xavier Sivecas). 
and became a YouTube favorite after songstress Björk purchased one for her 2007 world tour. Each physical token on the reacTable has a dedicated function, for example, generating sound, filtering audio, or controlling sound parameters. Visual programming becomes easier through dynamic patching, where compatible input and output "slots" automatically attract each other through proximity. The "foremost goal was to design an attractive, intuitive and non-intimidating musical instrument for multi-user electronic music performance" [124] that is engaging from the first minute but also is complex, subtle and allows for endless variation. Another commercially available system is the AudioCubes [209]. It consists of a handful of cubes that detect each other's vicinity and communicate with each other. Each cube sends and receives audio through its faces. The cubes also act as speakers and light up in colors according to their configuration. Block Jam [167] is a dynamic polyrhythmic sequencer built from cubes attaching to each other.

The Audiopad system [182] allows users to manipulate and mix sound samples by placing tangible tokens onto an augmented surface (see Figure 4.13). New samples can be dragged onto the surface from a menu on the rim. mixiTUI [183] is a tangible sequencer for sound samples and edited music, which adds loops, controls, and effects onto sounds, and utilizes interaction mechanisms (in particular dynamic patching) from the ReacTable.

Kaltenbrunner [129], on his overview website, distinguishes between the following types of tangible musical interfaces. Tangible musical artifacts have music "contained" within a sensorized object, and
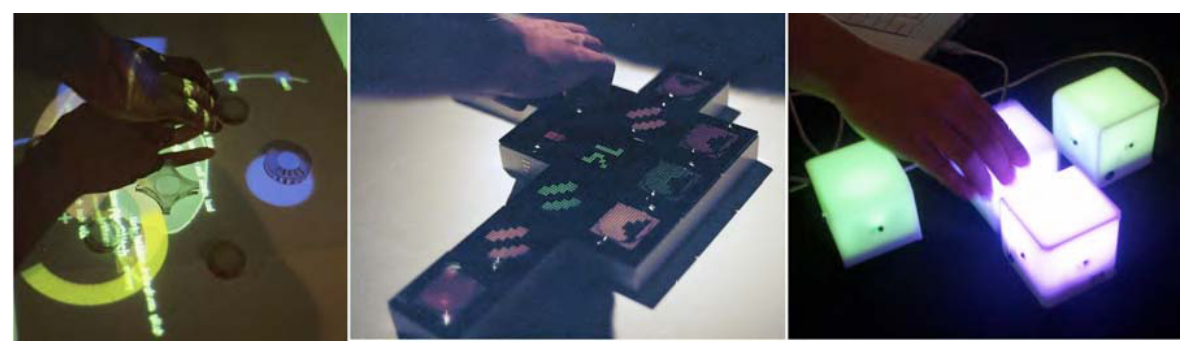

Fig. 4.13 From left to right: Audiopad [182], BlockJam [167], and Audiocubes [209] with the cubes glowing in green and white (photos by E. Hornecker). 
different interactions, like rubbing, squeezing, or plucking trigger a different replay (e.g., the Squeezables [250]). Musical building blocks (e.g., [167]) consist of blocks that continuously generate or manipulate sound and can be stacked, attached, or simply placed in each other's vicinity. With some systems, blocks work independently of each other, with others the spatial arrangement modifies sound, which is transmitted and processed through sequencing blocks. With token-based sequencers a surface is repeatedly scanned and each slice of the scan generates sound, location of a token, or other attributes like color determining the note or rhythm played. Furthermore, there are touch-based music tables and tangible music tables (e.g., AudioPad [182], reacTable [125]) which interpret interactions with tangible tokens on an interactive table surface. Finally, there are a range of commercial tangible music toys such as Neurosmith's MusicBlocks and Fisher-Price's play zone music table. These usually allow selection and placement of tangible objects into slots, activating associated sounds modified by the position and overall configuration of elements.

Many musical interfaces are aimed at entertaining and educating the public, as is the case with the Waltz dice game in the Vienna Haus der Music. Another emerging application area for TUIs related to music is performance. For example the Media Crate [13] supports VJ-ing, the live presentation of audio-visual media on multiple screens taking input from various sources. The system has a tabletop interface very similar to the reacTable. Through manipulation of tangible tiles on the table the VJ can order and preplan clips for the show, edit properties, display clips on a particular output, etc. Sheridan and Bryan-Kinns [222] discuss experiences with the uPoi, a performance instrument which is based on swinging an instrumented poi (a ball tied to a string) around one's own body, where the acceleration data is converted into audio and sound output. From performing at outdoor music festivals and enticing the audience to participate and play with the uPoi, design requirements for performative tangible interaction are derived: intuitive use, unobtrusiveness, enticingness (visibility of interaction and low entry threshold), portability, robustness, and flexibility of the setup. 


\subsection{Social Communication}

Since their early days, TUIs were employed as communication support tools. Tangible objects seem suited to represent people and can be positioned in the periphery of attention to support ambient awareness, while staying at hand. As one among several alternative interfaces for Somewire, an audio-only media space, tangible figurines could be positioned on a rack to determine audibility and directionality of sound [224]. The TUI was evaluated as more adequate for the task than two GUI-based prototypes. Greenberg and Kuzuoka [83] employed physical figurines as "digitally controlled physical surrogates of distant team members" for a video-conferencing system. The peek-a-boo surrogate for instance faces to the wall when the remote person is away, and rotates into view once activity is sensed at their desk (Figure 4.14). Moving the surrogates furthermore effects the fidelity of the transmission and thus serves as privacy mechanism.

A range of recent projects focus on remote awareness within social networks, for example of groups of friends as in the case of Connectibles [128], or distributed work groups [23] where physical objects
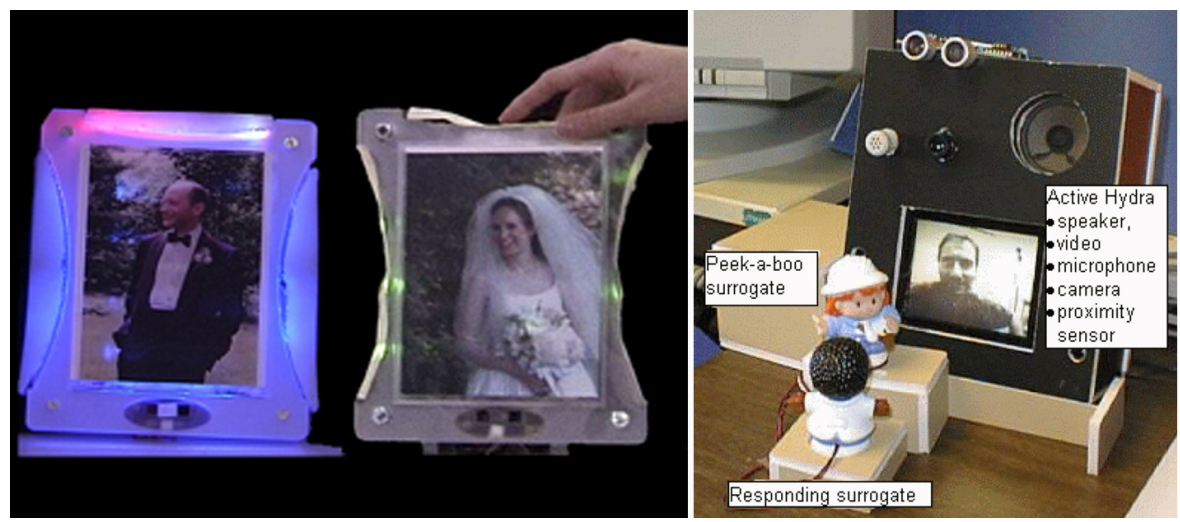

Fig. 4.14 Left: Lumitouch [34], a digitally augmented picture frame that allows intimate communication through touching a picture frame. A set of two frames is held by remote partners. When one frame is being touched, the other lights up (photo: courtesy of Angela Chang). Right: the peek-a-boo surrogates [83], one of the first TUIs supporting social communication. Positioning of the figurines indicates availability of other people within a networked media space and influences the fidelity of the connection (image courtesy: Saul Greenberg). 
transmit awareness information and abstract messages. Edge and Blackwell [51] employ tangibles for task management in office work, and highlight their potential for symbolic social communication, as handing over a token can represent handing on the responsibility for a task or document.

A range of prototypes addresses remote intimacy. In this context, researchers often experiment with different sensory modalities. For example, Strong and Gaver [228] present "feather, scent and shaker". Squeezing a small device when thinking of the other triggers feathers to fall down a tube, activating a scent, and shaking it makes the other device vibrate. LumiTouch communicates the action of touching a picture frame [34]. Partners can establish a simple vocabulary of touches, or touching may convey an abstract "I think of you". Lovers Cup [35] has a glass light up when the remote partner uses his/hers in support of the shared ritual of drinking wine together. InTouch [22] consists of two interconnected rollers that transmit movement, and is one of the earliest proponents of using the haptic or tactile modality for remote communication and intimacy. United Pulse [255] transmits the partner's pulse between two rings. Other projects transmit hugs or touches via networked clothing (falling into the domain of wearable computing). The Interactive Pillows project [53] lets a distant pillow light up with dynamic patterns when another pillow is touched, hugged, or pressed.

\subsection{Tangible Reminders and Tags}

Tangibles lend themselves to tagging and mapping applications where the tangible object is utilized to trigger digital information or functions. This use of digital linkages is somewhat related to the "internet of things" vision, but does not include autonomous and "intelligent" objects. Instead, it tends to require explicit interactions, such as placing a particular object in proximity of a reader.

Holmquist et al. [101] explore the use of physical tokens to bookmark and recall webpages. Want et al. [249] discuss a variety of scenarios where physical objects are digitally tagged, for example linking a physical book with an electronic document, a business card with a home page or a dictionary is tagged to invoke a language translation 
program. Van den Hoven and Eggen [242] and Mugellini et al. [165] investigate tangible reminders, where placing vacation souvenirs on a surface opens an associated photo collection. An overview by Martinussen and Arnall [158] shows that there is already a wide range of commercial applications and systems based on physical-digital tags, such as RFID-keyfobs and RFID tagged toys. They go on to explore and discuss the design space of embedded RFID tags in physical objects, taking account of issues such as the size of tags and the shape of the emitted field. 


\section{Frameworks and Taxonomies}

As the field matures, researchers have developed frameworks and taxonomies that aim to provide TUI developers with explanatory power, enable them to analyze and compare TUI instances, and apply lessons learned from the development of previous TUI instances to future efforts. Some frameworks may have a generative role, suggesting new directions to explore, and uncovering open opportunities in the TUI design space. Frameworks can be characterized as providing a conceptual structure for thinking through a problem or application. Thus, frameworks can inform and guide design and analysis. Taxonomies are a specific type of framework that classify entities according to their properties, ideally unambiguously.

In this section we review a range of frameworks and taxonomies for tangible interfaces. As the field has developed, a greater number of frameworks relevant for the broader context were proposed as well as domain-specific frameworks and frameworks that focus on the user experience. Such frameworks were rare (cf. [160]) in the early days of TUI research, as early work tended to focus on taxonomies and terminologies, analyzing potential mappings between the physical and digital, or investigated affordances and physical form. Our survey covers a 
different range of publications than Mazalek and van den Hoven's [160] overview of frameworks on tangible interaction (the broader context). However, similarly, we find that to date only few frameworks provide guidance or tools for building new systems.

\subsection{Properties of Graspable User Interfaces}

Fitzmaurice [65] defined a graspable user interface as providing a "physical handle to a virtual function where the physical handle serves as a dedicated functional manipulator". Users have "concurrent access to multiple, specialized input devices which can serve as dedicated physical interface widgets" and afford physical manipulation and spatial arrangement.

A core property of graspable user interfaces [65, 67] is spacemultiplexing, which is a very powerful concept. When only one input device is available, it is necessarily time-multiplexed: the user has to repeatedly select and deselect objects and functions. A graspable user interface on the other hand offers multiple input devices so that input and output are distributed over space, enabling the user to select an object or function with only one movement by reaching for its physical handle. This allows for simultaneous, but independent and potentially persistent selection of objects. Moreover, we can have dedicated functionally specific input/output devices which directly embody functionality [66]. For example, a user may select a few functions (physical blocks) for later use and arrange them as a reminder and as a "tangible plan". TUIs can be interpreted as a radical continuation of these ideas.

The speed and accuracy of manipulating graphical objects this way was tested in empirical experiments [66]. These revealed that space-multiplexing is effective by reducing "switching cost", exploiting innate motor skills, and hand-eye coordination. It may further offload demands on visual perception. Fitzmaurice [65] describes five basic properties of graspable interfaces, with the latter four enabled (but not necessitated) by the first:

- space-multiplexing,

- concurrent access and manipulation (often involving twohanded interaction), 
- use of strong-specific devices (instead of weak-general, that is generic and non-iconic),

- spatial awareness of the devices, and

- spatial reconfigurability.

A subsequent discussion of a range of systems makes evident that rating systems along these properties is not a clear-cut decision. Does a system need to be spatially aware under all circumstances or is it sufficient if the user keeps to certain rules? Is an iconic or symbolic physical form a core requirement for a graspable interface? What if the application area is intrinsically abstract and does not lend itself to iconic representations? Should concurrent manipulation always be feasible? How do we distinguish between the system concept and its technical implementation? Thinking about these properties probably serves as a useful thought exercise, helping to understand the properties and limitations of different systems, but should not result in in-out listings.

\subsection{Conceptualization of TUls and the MCRit Interaction Model}

In 2001, Ullmer and Ishii presented first steps toward identifying tangible user interfaces as a distinct and cohesive stream of research [238]. They highlight key characteristics and present an interaction model for tangible user interfaces. Ullmer and Ishii defined tangible user interfaces as systems that give physical form to digital information, employing physical artifacts both as representations and controls for computational media. This definition would later be broadened by emerging frameworks such as [63] and [105]. Drawing from the MVC (Model, View, Control) model of GUI-based interaction, Ullmer and Ishii suggest an interaction model called MCRit, an abbreviation for Model-Control-Representation (intangible and tangible). While the MVC model emphasizes the separation between graphical representation (i.e., view) and control (mediated by input devices such as a mouse and a keyboard), the MCRit model highlights the integration of physical representations and control in tangible user interfaces, which basically eliminates the distinction between input and output devices. 


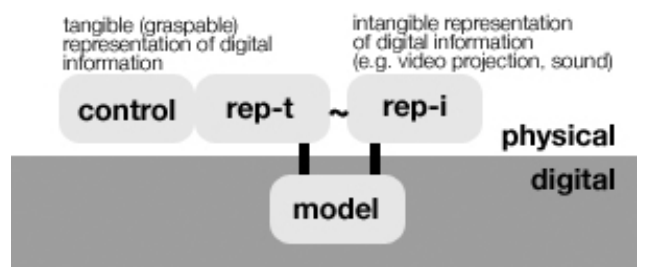

Fig. 5.1 The MCRit model (redrawn based on [238]) eliminates the distinction between input and output devices.

This "seamless integration of representation and control" means that tangible objects embody both the means of representation and the means of manipulating digital data. The MCRit model (Figure 5.1) illustrates three central relations, which translate to properties of TUIs. A fourth property results from integrating the first three:

- tangible objects are coupled via computerized functionality with digital data (computational coupling);

- the tangible objects represent the means of interactive control. Moving and manipulating objects is the dominant form of control;

- the tangible objects are perceptually coupled with digitally produced representations (e.g., audio and visuals); and

- the state of the tangible objects embodies core aspects of the entire system's state (representational significance). (the system is thus at least partially legible if power is cut).

\subsection{Classifications of TUls}

Ullmer et al. [239] identify several dominant approaches or types of TUIs:

- Interactive Surfaces. Frequently, tangible objects are placed and manipulated on planar surfaces. Either the spatial arrangement of objects and/or their relations (e.g., the order of placement) can be interpreted by the system. A typical example for interactive surfaces is Urp [241]. 

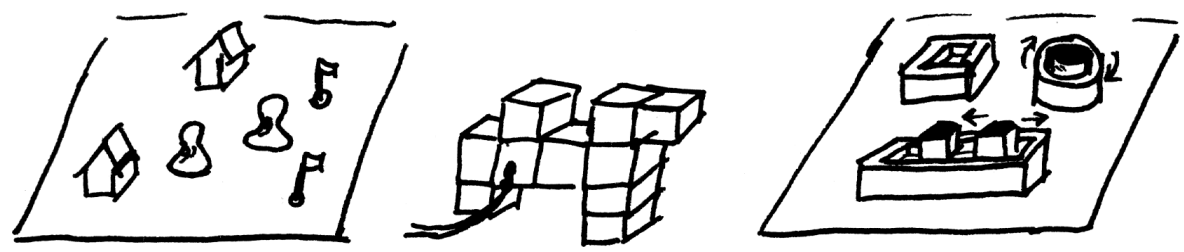

Fig. 5.2 The three dominant types of TUIs: tangible objects on interactive surfaces, constructive assemblies of modular connecting blocks, and token-constraint systems.

- Constructive Assembly. Modular and connectable elements are attached to each other similar to the model of physical construction kits. Both the spatial organization and the order of actions might be interpreted by the system. Typical examples for constructive assembly systems are the "intelligent 3D modeling" toolkits by Aish [3] and Frazer and Frazer [72], or as newer examples, BlockJam [167] and Topobo [196].

- Token+Constraint systems combine two types of physicaldigital objects. Constraints provide structure (stacks, slots, racks) which limit the positioning and movement of tokens mechanically and can assist the user by providing tactile guidance. The constraints can express and enforce the interaction syntax. Typical examples for this type of TUI are the Marble Answering Machine and the Slot Machine [185].

These classifications are not always evident how to apply. For example, tokens can act as a constraint for other tokens and constraints might be placed within other constraints. Many systems cross these categories, e.g., having constraints placed on an interactive surface. Moreover, in the case of SandScape [116] users shape the sand (or transparent plastic beads) in a box to alter the topography of a landscape model. The sand provides a continuous material, rendering the term "token" somewhat meaningless.

Sharlin et al. [219] identify the particular strength of TUIs in exploiting human experience of spatiality. Good design should thus employ successful spatial mappings, unify input and output spaces, and enable trial-and-error activity. Evidently, spatial mappings are the most natural when the application itself is inherently spatial. Sharlin 
et al. thus define spatial TUIs as a subset of tangible interfaces that "mediate interaction with shape, space and structure".

Van den Hoven and Eggen [242] and Edge and Blackwell [51] present examples for and argue for the relevance of non-spatial TUIs, where tokens are not interpreted as a spatial or relational ensemble. With van den Hoven's photo browser, placing a souvenir on the surface brings up associated photo's, constituting a one-to-many association. Edge and Blackwell [51] argue that when designing tangibles for dealing with abstract relationships it is better to not use spatial mappings (that is, to disregard spatial configuration) because the disadvantages in terms of the tangible correlates of cognitive dimensions outweigh the advantages. The use context of their system is characterized by strong space constraints and a high risk of accidental changes to the spatial configuration, creating further arguments for intentionally loose mappings. This peripheral interaction surface is furthermore interesting in terms of de-coupling representation and control (counter to the recommendations from Ullmer and Ishii [238]) by requiring the user to use the other hand to twist and push a knob, which makes interaction very explicit and prevents accidental changes.

\subsection{Frameworks on Mappings: Coupling the Physical with the Digital}

Several frameworks focus on the mappings or couplings between the physical world (objects and manual user input) and digital world (data, system responses). We first discuss approaches that categorize and introduce terminology for describing mappings. We then move on to frameworks that aim to provide a better understanding of mappings in order to improve user interaction with tangible systems.

Ullmer and Ishii identify the coupling of physical representations to underlying digital information and computational models as a central characteristic of tangible interfaces [238]. They recognize a wide range of digital information classes that could be associated with physical objects. These classes include: static digital media such as images and 3D models; dynamic digital media such as live video and dynamic graphics; digital attributes such as color or other material properties; 
computational operations and applications; simple data structures such as lists or trees of media objects; complex data structures such as combinations of data, operations, and attributes; and remote people, places, and things (including other electronic devices). They also highlight two methods of coupling objects with information:

- static binding that is specified by the system's designer and cannot be changed within the tangible interface itself; and

- dynamic binding that is specified within the tangible user interface, typically by the user of the system.

Several taxonomies for TUIs further examine the coupling of physical objects to digital information. Holmquist et al. introduce a taxonomy of physical objects that can be linked to digital information [101], suggesting three categories of objects: containers, tokens, and tools. Containers are generic objects that can be associated with any type of digital information and are typically used to move information between platforms. Tokens are physical objects that resemble the information they represent in some way, and thus are closely tied to the information they represent. Tokens are typically used to access information. Finally, tools are used to actively manipulate digital information, usually by representing some kind of computational function. Ullmer and Ishii [238] suggest a slightly different taxonomy, where token is a generic term for all kinds of tangible object coupled with digital information, and containers and tools are subtypes. This terminology has the advantage of allowing for different semantic levels of meaning for one object, for example, a token representing a van has a distinct and iconic identity and also is used to move different entities around.

A number of more recent frameworks analyze the nature of mappings in more detail, with the aim to improve user interaction with tangible systems. Tight mappings can provide the user with the feeling of direct control and create the impression of unified physical-digital objects. An important contribution of these frameworks is the extension of the notion of mappings beyond Norman's purely spatial "direct mapping".

Fishkin [63] suggests two axes, metaphor and embodiment, as particularly useful when describing and analyzing tangible interfaces. 
Fishkin's embodiment axis represents how closely the input focus is tied to the output focus in a TUI application, or in other words, to what extent does the user think of the state of computation as being embodied within a particular physical housing. When a system seeks to maximize the direct manipulation experience, the level of embodiment should be high. However, when mapping between physical representations and digital information is more abstract, indirect coordination of input and output is often used. There are four levels of embodiment:

- full where the output device is the input device,

- nearby where the output takes place near the input object,

- environment where the output is around the user, and

- distant where the output is on another screen or even in another room.

The second axis, metaphor, describes the type and strength of analogy between the interface and similar actions in the real world. Fishkin groups metaphors into two types and argues that the more either type is used, the more tangible the interface is:

- metaphors of nouns, that appeal to the shape of an object,

- metaphors of verbs, that appeal to the motion of an object or its manipulation.

Koleva et al. [142] present an analytic framework that inquires into how the links between the physical and the digital can be made intelligible for users. The properties of links between physical and digital objects influence to what extent these are perceived as the same thing or as two separate, but connected objects (level of coherence). These properties include the relation between physical action and digital reaction (literal or transformed effects), how much of the interaction is sensed and transmitted, the duration and configurability of the connection, the autonomy of the digital object (the extent to which the existence of a digital object depends on a physical object), the cardinality and the directionality of links. Five categories of interfaces along the coherence continuum are identified: general purpose tools, specialized tools, identifiers, proxies, and projections. 


\section{Frameworks and Taxonomies}

Finally, Interaction Frogger [254] brings a product design perspective to tangible interaction design. This framework analyzes personproduct interactions in terms of the coupling between a person's action and the product's function, reaction, and information output. It identifies six aspects of natural coupling: time, whether the user action and product reaction coincide in time; location, whether they coincide in space; direction, whether the direction of the user's movement is similar to that of the product's reaction; dynamics, whether the dynamics (position, speed, acceleration, force) of the user's action is coupled to the dynamics of the product's response; modality, whether the sensory modalities of action and product reaction are similar; and finally expression, whether the product's reactions reflects the emotional expression of the input action.

The framework also identifies three types of information in interaction with a product: functional information is a direct result of the product's function (the oven door swings open on pulling the handle down), augmented information informs the user about the internal state of the product (an LED lights up to indicate the oven warms up), and inherent information results directly from the user's action (e.g., the feeling of a button pressed down and hearing it clicking). Unification along the six listed aspects provides the impression of a natural coupling. If the system functionality does not allow for a direct coupling (e.g., a remote control is by nature distant in location) or if functional feedback will be delayed, designers can add intermediate levels of feedback and of "feedforward" (upfront information about the outcome to be expected from an action) in the form of inherent or augmented information to restore perceptible relations between action and reaction, and to guide users' actions. This framework provides guidance for design through a systematic step-by-step approach that analyses each aspect of couplings and looks for ways to substitute functional information first through inherent feedback/forward and then by augmentation.

\subsection{Tokens and Constraints}

While previously discussed frameworks are focused on mapping physical form to digital information, the TAC paradigm [216] is concerned 
with identifying the core elements of a TUI. The TAC paradigm introduces a compact set of constructs that is sufficient for describing the structure and functionality of a large subset of TUIs. This set of constructs aims to allow TUI developers to specify and compare alternative designs while considering issues such as form, physical syntax, reference frames, and parallel interaction.

Drawing upon Ullmer's Tokens+Constraints approach [239], Shaer et al. [216] describe the structure of a TUI as a set of relationships between physical objects and digital information. They identify four core components that together could be combined to describe the structure and functionality of a TUI. These include: Pyfo, Token, Constraint, and TAC.

A pyfo is a physical object that takes part in a TUI (e.g., a surface, a building model, a block). Pyfos may enhance their physical properties with digital properties such as graphics and sound. There are two types of pyfos: tokens and constraints. Each pyfo can be a token, a constraint, or both.

A token is a graspable pyfo that is bound to digital information or a computational function. The user interacts with the token in order to access or manipulate the digital information. The physical properties of a token may reflect the nature of either the information or the function it represents. Also, the token's physical properties may afford how it is to be manipulated.

A constraint is a pyfo that limits the behavior of the token with which it is associated. A constraint limits a token's behavior in the following three ways: (1) the physical properties of the constraint suggest to the user how to manipulate (and how not to manipulate) the associated token. (2) the constraint limits the physical interaction space of the token; and (3) the constraint serves as a reference frame for the interpretation of token and constraint compositions.

Finally, a TAC (Token and Constraints) is a relationship between a token and one or more constraints. TAC relationship often expresses to users something about the kinds of interactions an interface can (and cannot) support. TAC relationships are defined by the TUI developer and are created when a token is physically associated with a constraint. Interacting with a TAC involves physically manipulating a token (in a 
discrete or a continuous manner) in respect to its constraints. Such interaction has computational interpretation. The manipulation of a token in respect to its constraints results in modifying both the physical and digital states of the system. Thus, Shaer et al. [216] view TAC objects as similar to Widgets because they encapsulate both the set of meaningful manipulations users can perform upon a physical object (i.e., methods) and the physical relations between tokens and constraints (i.e., state).

To specify a TUI using the TAC paradigm constraints, a TUI developer defines the possible TAC relationships within a TUI. This defines a grammar of ways in which objects can be combined together to form meaningful expressions, expressions that can be interpreted both by users and the underlying computational systems. Shaer et al. demonstrated the TAC paradigms' ability to describe a broad range of TUIs, and have shown that the set of constructs it provides is sufficient for specifying TUIs classified as interactive surfaces, constructive assemblies, and Token + Constraints systems [239], as well as additional interfaces outside these classifications. The TAC paradigm constructs also laid the foundation for TUIML, a high-level description language for TUIs [215](see Section 8.1.3).

\subsection{Frameworks for Tangible and Sensor-Based Interaction}

Several frameworks have emerged to provide a conceptual understanding of systems within the broader context of TUIs, for example on sensor-based and tangible interactions.

\subsubsection{Sensor-Based Interaction}

Bellotti et al. [16] describe six challenges for interacting with sensing systems. They analyze interaction in analogy with communication, focusing on problems arising from invisible computing and implicit control. Imagine you converse with an invisible partner that observes all of your actions, reacts to anything it interprets as a command, but cannot talk back to you. The challenges comprise identifying and addressing the system (where do I turn to explicitly address the system, how do I turn away from it?), issuing control commands unambiguously, 
including complex ones, monitoring (availability of feedback which conveys whether the system attends to me and how it interprets the command), and recovery from errors (reversibility).

TUI input is often explicit. To use a TUI, one needs to manipulate a physical artifact and usually receives tactile and visible feedback. Once the user knows how to address the system, (s)he can avoid unintended address and selection, and is thereof aware of system response or lack of response. Tangible interfaces ease the a priori identification and selection of actions, especially with strong-specific, dedicated tokens, where shape and look suggest meaning. Specification of complex commands (abstract, concatenated, or acting on a set of objects) generally is a weak point of all direct manipulation interfaces. TUIs are further weak in undoing mistakes as there is no undo function; the previous system state has to be remembered and manually reconstructed.

Benford et al. [17] focus on which interactions can be sensed and which cannot, and on what actions users might do or desire to do. Their framework asks designers to explicitly compare possible and likely user actions while considering unexpected user behaviors, the system's sensing capabilities, and desirable effects. Actions that can be sensed, are expectable, but do not make sense in a literal way, could be mapped to desired functionality or effects that cannot be achieved with a literal, direct mapping. As example, they describe a telescope on wheels that provides an augmented view of the terrain and gives a bird's-eye view, slowly flying up, when pointing downward. This could be a useful strategy in TUI design for extending system functionality beyond simple mappings (cf. [105]) and preventing user input that the system cannot interpret.

Rogers and Muller [200] discuss the user experience of sensor-based interaction. Instead of viewing the uncertainty and lack of control that are often associated with sensor-based interaction as problems to be overcome, they suggest interpreting these qualities as opportunities to be exploited. Designers might intentionally employ ambiguous couplings between user action and effects to create puzzling experiences that impel people to reflect. This can be especially useful for play and learning activities, where uncertainty (and mastering it) may be an integral part of the experience. They identify three core dimensions 
of sensing: discrete-continuous (e.g., a button press versus gestures), the sensors degree of precision, and whether interaction is explicit or implicit. They further suggest that for play activities, a certain degree of unpredictability and imprecision can increase creativity, reflection, and enjoyment.

\subsubsection{Tangible Interaction}

Hornecker and Buur's [105] Tangible Interaction Framework focuses on the user experience, and in particular the social interaction with and around tangible systems. As described earlier in Section 3.2.2, tangible interaction is defined as a broader area that encompasses TUIs. Tangible Interaction encompasses research on whole-body interaction, interactive spaces, and gestural input methods. In particular, it shifts attention from the visible interface to the interaction and to how users experience this interaction. This shift makes the positioning of tangible interfaces as an antithesis to graphical interfaces in the early publications [117] (which was useful as a provocative statement and driving force for research) obsolete - tangible interaction may well combine graphical and tangible elements. It further directs attention toward the qualities of interaction with the system, and away from its technical functioning.

Hornecker and Buur [105] identify four core themes that should be considered when designing or assessing tangible interaction for use scenarios that have social aspects. These themes are then broken down into concepts and sensitizing questions.

- Haptic Direct Manipulation refers to the material qualities and the manual manipulability of these interfaces;

- Spatial Interaction refers to their spatial qualities, including whole-body interaction and the performativeness of interacting in space;

- Embodied Facilitation refers to how physical setup and digital programming can predetermine and guide patterns of use; and

- Expressive Representation refers to the expressiveness and representational function of tangibles. 
This focus on the user experience and contextual embedding of interaction is an example of the contemporary trend in research that Fernaeus et al. [58] describe as a "participant's perspective on action and interaction with technology". They identify four emerging themes (shifts of ideals), which correspond to the so-called "practice turn" within social and cognitive sciences. First, while the initial definitions of TUIs focused on representation and transmission of information, newer conceptualizations instead focus on human action, control, creativity, and social action (cf. [105, 124]). The second theme is a focus shift from system functionality toward the physical and social context of interaction with and around the system. The third theme is that focus has shifted from supporting individual to social interaction. Finally, subjective interpretations and non-intended appropriation of tangibles have become a research theme. With these changes in perspective, designers are increasingly interpreting tangibles as "resources for action" and intentionally supporting "offline interaction", which is directed at the social/physical setting instead of at the computer, and therefore is not tracked by the system.

\subsection{Domain-Specific Frameworks}

\subsubsection{Tangibles and Learning}

The area that has seen the greatest boom of domain-specific frameworks is certainly learning (see [174] for a detailed overview). Several frameworks categorize tangible interfaces in this domain according to the types of activities they promote. For example, Marshall et al. [157] distinguish expressive and exploratory tangibles. Expressive tangibles allow the learners to create their own representations and the system becomes a tool. Exploratory tangibles provide users with a model that they try to understand. Focusing on Digital Manipulatives as a species of TUIs that builds on educational toys such as building blocks, Zuckerman et al. [266] propose to classify these according to whether they foster modeling of real world or the construction of abstract and generic structures. They propose guidelines for the more abstract "Montessoriinspired-Manipulates", including the use of generic objects, specific 
semantic associations (e.g., mathematical operations), and encouragement of analogy (ability to annotate blocks).

Scaife et al. investigated the theme of couplings, or "transforms", in the context of digitally augmented learning and play activities [192, 193, 201]. Their hypothesis is that combining familiarity with unfamiliarity promotes creativity, inquisitiveness and reflection. The transforms between physical to digital and between digital to physical are assumed to be less familiar to learners, and hence motivating learners to figure out what causes them. On the other hand, the transforms between digital to digital and between physical to physical are considered familiar. Rogers et al. [201] proposed a conceptual framework of mixed realities that are categorized along these four possible transforms. They designed a range of open-ended activities that allowed children to experience different transforms between actions and effects. Overall, they found that physical interaction and unfamiliarity resulted in more communication among children and more theorizing about what was happening in the system. Such interactions thereby led to more reflection and exploration.

Some of the more recent frameworks attempt to provide a structured overview of issues relevant for tangible learning systems, to provide guidance on the cognitive and social effects of learning with tangible interfaces, and to point out research avenues.

The Child Tangible Interaction (CTI) framework [6] is an explanoratory conceptual framework that derives abstract design guidelines for tangible and spatial interactive systems from the literature on children's development of abstract cognitive structures. It describes five aspects of interaction: systems as spaces for action, perceptual, behavioral, and semantic mappings, and how systems can provide "space for friends" by supporting collaboration and imitation-behavior. It recommends to employ body-based interaction, to support epistemic action, and to consider age-related perceptual, cognitive, and motor abilities, and children's understandings of cause and effect relations. The CTI framework further highlights how leveraging children's body-based understanding of concepts and spatial schema for more abstract concepts can provide learning opportunities. 
Marshall [156] reviews the literature in search of arguments and knowledge about how tangible interfaces support learning. The paper identifies six "latent trends and assumptions", and outlines a series of open research questions. These relate to: learning benefits, learning domains, types of activity, integration of representations, concreteness and sensory directness, and effects of physicality on learning. Marshall criticizes that often information could just as well be presented graphically rather than tangibly, and that evaluations of TUIs often do not address the specific contribution of tangibility in terms of which elements of the TUI design are critical for learning. Furthermore, he argues that concreteness and physicality need to be distinguished (e.g., physical artifacts can be abstract), and points out potential negative side-effects of concrete representations, as these can result in decreased reflection, less planning and learning. Most importantly, this metaanalysis of the research area highlights the need to empirically demonstrate measurable benefits of physical manipulation for learning.

Price [191] starts to tackle the question of learning benefits, and interprets tangibles as representational artifacts that may be coupled with other representations. This framework supports a systematic investigation of how different couplings between digital information and physical artifacts influence cognition. These associations can be compared along location (separated, co-located, or embedded location of input in relation to output), the dynamics of coupling (perceived causality, intentionality of actions), the artifacts' correspondence to the object domain in terms of metaphors and handling properties (e.g., fragility), and modality. Price then employs the framework in investigating how different representational relations influence inference and understanding. 


\section{6}

\section{Conceptual Foundations}

In this section we provide an overview of the conceptual background that informs research on and design of tangible interfaces. This chapter begins by discussing how research on affordances and image schemas can inform TUI design and how TUI research has been inspired by theories of embodied and situated interaction. We then review how theories of external representations and distributed cognition can apply to TUI design and review studies of two-handed interaction. We conclude with perspectives from the field of Semiotics, the study of signs and meaning.

\subsection{Cuing Interaction: Affordances, Constraints, Mappings and Image Schemas}

Frequently, descriptions of tangible interfaces and arguments for their advantages refer to the notion of affordance. "Our intention is to take advantage of natural physical affordances to achieve a heightened legibility and seamlessness of interaction between people and information" [117].

Gibson's notion of affordance [76] has been introduced to HCI by Donald Norman [168]. Affordances denote the possibilities for action 
that we perceive of an object in a situation. Norman discusses them as properties of an object that invite and allow specific actions - a handle affords holding and turning, a button affords pressing. Later on [170], he distinguishes between perceived affordances which are only visually conveyed and rely on interpreting images, e.g., buttons on a GUI, and real (i.e., physical) affordances. Evidently, the power of TUIs lies in providing both real and perceived affordances. Product designers have enriched the discussion about affordances by pointing out that physical objects might not just invite, but moreover seduce us to interact via "irresistibles" that promise aesthetic interactions [176].

A careful investigation of object affordances, for example studying the ways in which people hold and handle differently shaped objects, can guide the design of physical forms for tangible interfaces (cf. [221, 62]). Variations in size, shape, and material of an object as simple as a cube affect the ways in which users handle it. We will return to the topic of affordances in Section 9.

Norman discusses constraints that restrict possible actions in tandem with affordances. Constraints physically prevent certain actions or at least increase the threshold for an action. For example, a flap that has to be held up to access a row of buttons underneath is considered a constraint. Combined together, constraints and affordances can be used to guide users through sequences of action [49].

The Token and Constraints approach [216, 239] explores physical and visual guidance to movement of loose items. In Norman's design theory, affordances and constraints were further supported by the notion of mapping, that is the visual relations between intended actions and effects, and between interface elements and the related output realm. The simplest example is the arrangement of oven knob controls. So-called natural mappings employ spatial analogies and adhere to cultural standards. Unfortunately with today's complex devices, such natural mappings often do not exist as interfaces need to abstract away from physical layouts of the application domain and complex action sequences are controlled [49]. TUI design, if aiming for powerful functionality, thus needs to find new solutions to provide legible mappings.

Hurtienne and Israel $[111,112]$ have recently introduced a potential approach that can underpin the design of legible mappings by 
presenting an empirical analysis of the utility of image schemas [123]. This work has shown how sensorimotor knowledge, which is prevalent in linguistic metaphors [145], can be productively utilized in interface design and provide a basic vocabulary for mapping. Essentially, image schemas [111] consist of relatively simple and abstract relations such as "up is more and down is less" that are based on embodied experience. An interpretation such as "up is better" exemplifies a metaphoric extension. Other important schemas that are metaphorically extended in non-linguistic reasoning are path, containment (in-out), force, and attribute pairs like heavy-light, warm-cold, or dark-bright. Based on embodied experience, they are learnt early in life, shared by most people and processed automatically. Violating the metaphorical extensions results in increased reaction times and error rates. Since image schemas are multimodal, tangibles seem particularly suited for making use of them (cf. [112]). For example, path and containment relations can be directly represented in the interaction with a TUI through actual physical movement (moving a token along a path and into a box) and tangible objects can be designed to convey attributes such as weight, temperature, or color which can be directly perceived.

\subsection{Embodiment and Phenomenology}

The notion of embodiment has been influential in many ways during the history of Tangible User Interfaces, although not always explicitly. "Embodiment" refers to the fact that we are incarnated, physical beings that live in a physical world. Thus, humans are not abstract cognitive entities (the Cartesian view of cognition), but our bodies and active bodily experiences inevitably shape how we perceive, feel, and think.

The term embodiment is used in many different ways in the literature. Its simplest use refers to the physical embodiment of data and its control via physical body movement and devices. Norman [171] summarizes this trend which is only loosely related to philosophical and cognitive theories of embodiment under the term Physicality. While Embodiment is most strongest connected to the philosophical school of Phenomenology (exemplified in the writings of Martin Heidegger [91], Maurice Merleau-Ponty [162], and Alfred Schutz), over the last 
two decades there has been a general resurge of "embodiment", especially within the field of Embodied Cognition which spans cognitive science, linguistics, and philosophy, and has been influenced by American Pragmatism. Influential authors are, for example, George Lakoff and Mark Johnson [145] (cf. image schemas), Andy Clark [36], and Antonio Damasio [44]. Embodiment is studied in terms of how the form of the human body and bodily activity shape cognitive processes and language, the interaction of action and perception, and of perspective as an embodied viewpoint (see [202]). The term further is used referring to human situatedness in social and cultural practices, a position that relates to Merleau-Ponty's and Heidegger's emphasis on world as habitat and the primacy of being (Dasein) [202]. Phenomenology emphasizes the lived experience of having (and inhabiting) a body, the primacy of experience and perception, the intentionality of perception, and how we experience other living bodies as different from inanimate objects, enabling human intersubjectivity.

Theories of embodiment are often utilized in conceptual discussions of tangible interfaces (or tangible interaction) [6, 50, 59, 105, 111, 138]. Implicitly, the notion of embodiment is present from the very early Tangible Bits papers [117] which argue for a rediscovery of the rich physical aesthetics of manual interaction with beautifully crafted instruments and for bridges over the divide between the physical world that we inhabit and the digital world. For tangible interfaces, besides the exploitation of physical affordances, creating rich tactile experiences has thus been one of the driving ideas. The sense of touch is our primal and only non-distal sense - touching results in being touched. From an anthropological and phenomenological perspective, touch reminds us of our corporeal existence and vulnerability. Furthermore, tangible interfaces allow us to utilize our manual, and more general, bodily intelligence (cf. [257]), supporting skilled action [47, 138] cognitive development, and learning [6].

Ideas from situated cognition, situated action and phenomenology, were influential in Weiser's early argumentation for Augmented Reality and Ubiquitous Computing: "humans are of and in the everyday world" [251], a phrase that reminds of phenomenological ideas of habitation, Dasein and lifeworld. Dourish [50] expatiates this in his book "Where 
the Action Is - The foundations of Embodied Interaction", which made embodied interaction a common term within HCI. He defines embodied phenomena as those "that by their very nature occur in real time and space". Tangible computing aims to manifest computing in physical form, thereby making it part of the everyday world. But embodiment is not merely a physical manifestation; embodied interaction is grounded (and situated) in everyday practice and describes a direct and engaged participation in a world that we interact with. Through this engaged interaction meaning is created, discovered, and shared.

Dourish's analysis thus goes beyond tangibility - embodied interaction starts as soon as we engage with the world. The question is how systems can support this, and how they can co-inhabit and participate in our lifeworld. Interaction with objects can be at several levels of meaning. When we use them while focusing on the act of working and the desired goal, they are ready-to-hand, and when we start to focus on the object itself and how to interact with it, it becomes present-at-hand. As an example, Dourish provides an interesting analysis of the Illuminating Light system [240] in terms of embodiment and the multiple levels of meaning of moving the tangible blocks. These can be seen as metaphorical objects, as tools for changing the laser beam, as tools for interacting with a mathematical simulation, or as blocks to be cleared off the table, depending on what the intentional object of manipulation is. What the tangible interface allows for is a seamless combination of these levels of interaction. Representations become artifacts that can also be acted upon. Dourish further emphasizes embodiment as situatedness in social and cultural contexts, arguing that social computing, by tapping into our social lifeworld, builds on embodiment.

\subsection{External Representation and Distributed Cognition}

Describing how someone explains a car accident using at-hand artifacts to visualize the relevant objects, Donald Norman [169] explains how physical objects come to be used as graspable symbols which externalize our thinking and thereby help us think: "We can make marks or symbols that represent something else and then do our reasoning 
by using these marks. (... They) help the mind keep track of complex events. (This) is also a tool for social communication."

Theories of external representation [207] share this emphasis of the mind as enhanced and augmented by outside representations with the theory of distributed cognition [113, 98]. While distributed cognition tends to view mind and world as one larger system (arguing that parts of cognition occur in the world and between actors and artifacts), analysis of external representations tends to focus more on how people actively employ outside representations to their advantage.

Various studies have demonstrated how physical artifacts support cognition by serving as "thinking prop" and memory support. Probably, most well known are Kirsh's [136, 137] investigations of epistemic actions. These do not directly contribute toward the overall goal (they are not functional), but help exploring options, keeping track of previous paths taken, and support memory. Actions such as pointing at objects, changing their arrangement, turning them, occluding them, annotating, and counting all recruit external elements (which are not inside the mind) to decrease mental load. They manipulate the perceived (or real) action space to create constraints, hide affordances, highlight elements that can serve as future triggers for action, and thus reduce the complexity of activities. A part of human intelligence seems to lie in the use of strategies that decrease working memory load, direct attention, limit the search space, externally store information, and reduce the likelihood of error.

Directly visible constraints limit the search space and thus can decrease the need for explicit rules (e.g., Norman's example of the tower of Hanoi game). Spatial representations such as the relative length of parallel lines or the angle of a fuel tank needle can be directly read by human perception without requiring explicit logical deduction. This is referred to as perceptual intelligence or conceptual inference [114, 207]. The spatial nature of Tangible Interfaces can support such perceptual inferences, in particular when reading transitive, symmetrical, and asymmetrical relations.

Interfaces that make epistemic actions easier thus support cognition. Interfaces that limit interaction to actions of functional consequence may make the task harder to achieve. Tangible interfaces tend 
to allow for a wider range of actions and for more differentiated action (e.g., slightly angling a token to make it dance out of line) and are often referred to as supporting epistemic action and cognition. Patten and Ishii [180] compared the spatial memory and strategies of subjects sorting objects using a tangible interface and a graphical interface. They found that more epistemic actions were performed with the TUI and recommend allowing for what has come to be referred to as "out of bands interaction" with tangibles (cf. [41, 57]). Kim and Maher [134] conducted a comparative study of a GUI and TUI for a design task, and found a clear performance benefit for the TUI: the tangible $3 \mathrm{D}$ blocks allowed a rich sensory experience, which, by off-loading designer's cognition, promoted visuo-spatial discoveries, and inferences. The TUI also increased immersion, while facilitating interpretation and ideation. In TUI sessions more alternative ideas were proposed and elaborated, objects were moved more often in a trial-and-error fashion, more spatial relationships were discovered, a larger, more expressive set of gestures was used, and designers reformulated the design problem more often, alternating more between problem analysis and solution generation.

An important point about external representations is that they can be scrutinized much better than purely mental representations - they become an external object that its creator can distance itself from and see anew. Externalizing something forces to make fuzzy ideas concrete and can thereby uncover gaps and faults. Theories of design [211] describe this as the "backtalk" of the representation, with the designer engaging in a dialog with his/her own sketch.

The evidence on whether the properties that enable interactive exploration are supportive for learning is not conclusive (cf. [174]). Very "transparent" interfaces sometimes lead to less effective problem solving whereas systems that require learners to preplan their actions and to engage in mental simulations may result in better learning outcomes. Learning applications therefore often aim to encourage learners to reflect and abstract. For example, a study of children learning about numerical quantities found how different representations (sketching on paper versus physical manipulatives) increased the likelihood of different strategies to be discovered [153]. Manches et al. [153] conclude that 
digital augmentation might be exploited to encourage useful strategies. A distributed/external cognition analysis thus should not be limited to the physical realm, but needs to consider potential digital augmentation.

\subsection{Two-Handed Interaction}

Understanding the structure of bimanual manipulation is important for defining appropriate two-handed tangible interactions. A theoretical basis for the understanding and design of two-handed interaction exists in the form of Guiard's Kinematic Chain theory [84] and in humancomputer interaction studies $[9,31,67,89,143]$ that have explored cooperation of the hands.

Guirad's theory [84] and related studies highlight the ways in which the two hands are used in an asymmetric, complementary fashion, with the non-dominant hand often establishing a reference frame within which the dominant frame operates, and stabilizing this frame. Hinckley et al. [96] extend these ideas in their discussion of bimanual frames of reference and demonstrate that the non-preferred hand is not merely a poor approximation of the preferred hand, but can bring skilled manipulative capabilities to a task, especially when it acts in concert with the preferred hand. Often, the non-dominant hand acts in supportive anticipation of the actions of the other hand.

While early HCI studies on two-handed interaction viewed twohanded input as a technique for performing two subtasks in parallel [31], later studies showed that two-handed interaction provides additional benefits in the context of spatial manipulations [89] and 3D input [95]. Hauptmann showed that people often express spatial manipulations using two-handed gestures. Hinckely et al. [95] found that for 3D input, two-handed interaction can provide additional benefits:

(a) users can effortlessly move their hands relative to one another or relative to a real object, but moving a single hand relative to an abstract 3D space requires a conscious effort;

(b) while one-handed 3D input can be fatiguing, two-handed interaction provides additional support. When hands can rest 
against one another or against a real object, fatigue can be greatly reduced; and

(c) using two hands, a user can express complex spatial relations as a single cognitive chunk. This not only makes the interaction parallel (as opposed to being sequentially moded), but also results in an interface which more directly matches the users task.

Hinckly et al. [96] note "In related experimental work, we have demonstrated that using two hands can provide more than just a time savings over one-handed manipulation. Two hands together provide the user with information which one hand alone cannot. Using two hands can impact performance at the cognitive level by changing how users think about a task: using both hands helps users to reason about their tasks."

It has to be acknowledged that multi-touch surfaces also allow for two-handed interaction, but, as Kirk et al. [135] find in their reflection of experiences in building hybrid surfaces, true 3D manipulation is a core advantage provided by tangibles - many 3D actions are impossible without the third dimension on a surface.

\subsection{Semiotics}

Semiotics is the study of signs and the ways in which meaning is constructed and understood from signs. In semiotics, a sign is defined as anything that stands for something else to some interpreter [184]. Peirce, an American scientist, pragmatist philosopher and one of the founders of Semiotics, classified signs into thousands of categories, but acknowledged that the three most fundamental sign divisions are the icon, index, and symbol [184]. If a sign resembles, or in some way imitates the object it represents then the sign can be interpreted as being iconic. If a sign creates a link between the sign and an object in the mind of the perceiver (i.e., the perceiver must perform a referential action) then the sign is considered indexical (e.g., a smoke cloud is an index of fire). Finally, if a sign is based on convention that must be learned by the perceiver then the sign is symbolic. A sign may belong to more than one category. For example, a photograph can be considered 
an icon, because it looks like the object it represents. It is also an index of an event that has taken place at some point in time. Semiotics further differentiates between syntax (how signs can be arranged together according to grammar), semantics (what signs refer to), and pragmatics (how signs are used practically).

This Peircean model serves as a basis for studying computer-based signs (i.e., icons) and human-computer interactions. For example, Familant and Detweiler discuss attempts at taxonomies for GUI icons [55]. De Souza [45] presents the Semiotics Engineering process that views human-computer interactions as a communication process that takes place through an interface of words, signs, and behavior. She applies semiotics models and principles to help designers to tell users how to use the signs that make up a system. Finally, Ferreira et al. [60] present a semiotic analysis that can help designers reason about the changes they make when redesigning an interface.

In product design, semiotics has been influential [27], as designers attempt to consider what denotations and connotations different products will raise for consumers. For example, while a chair is for sitting, its material, shape, and size also denote a position in hierarchy (e.g., a throne), indicate whether it is for formal or casual use, have cultural associations (showing the owner is conscious of design) or serve as a status symbol (an expensive designer chair). Product semantics seeks to design such symbolic functions that contribute to the meaning of objects. Different designs with the same basic functionality then might for example appeal to different user groups. Product semantics often includes use of metaphor or visual analogy. This was often overdone (especially in product design of the 1980s), resulting in products overloaded with metaphors and meanings that users soon become weary of. Indeed, Durell Bishop, designer of the Marble Answering Machine that has been one of the major inspirations for the concept of TUIs (see page 12), proposed a completely different approach, focusing on the utility of affordances to support action and on different kinds of mappings.

As meaning is constructed within a larger sign system, where one sign might reference another, meaning tends to depend on the overall context. This means that different signs can have different meaning 


\section{Conceptual Foundations}

within different cultural contexts. Thus the meaning of signs can change through history. A design might further play with different connotations, and reference historic styles or contexts.

From a semiotic perspective, a TUI can be viewed as a system of signs where meaning is created by combining and manipulating signs, while tangible interaction can be viewed as a process of communication between designers and users. Given the rich form, material and contextual attributes of physical artifacts, semiotics offers a compelling viewpoint and conceptual tool for thinking about tangible representation and defining the relationship between designer and user. 


\section{Implementation Technologies}

To date, there are no standard input or output devices for TUIs. TUI developers employ a wide range of technologies that detect objects and gestures as well as sense and create changes in the real physical world. Strategies employed throughout the short history of TUI development range from using custom-made electronics and standard industry hardware to scavenging electronic devices or toys.

The difficulties in building a functioning TUI in the early days are hard to imagine nowadays. Standard industry microprocessors had to be used, which required rather low-level programming. Frazer et al. [71] built customized electronics hardware for their connectible blocks. The MIT Tangible Media Group (TMG), wanting to accurately detect the location of multiple tokens on a sensitive surface, took to scavenging electronic toys. Interactive toys from Zowie Intertainment used a mat woven of RFID antennas as a surface on which play figurines could be moved around in front of a computer screen. When Zowie abandoned the product, the TMG bought as many toys as possible on ebay. While the mats allowed precise location detection of up to $4 \mathrm{~mm}$, they could only differentiate nine electronic tags. Ferris and Bannon [61] utilized the circuitry of musical birthday cards to detect the opening of cardboard boxes. This kind of scavenging is still popular in the arts and 


\section{Implementation Technologies}

design community, where as part of the maker culture [175] it enables designers to build innovative prototypes cheaply. Yet, nowadays better toolkit and hardware support are available, easing development through high-level programming languages and purpose-built boards.

The breadth of technologies, devices, and techniques used for prototyping and implementing TUIs can be bewildering. Thus, we use a number of organizing properties to discuss and compare common TUI implementation technologies. Following, we describe three implementation technologies that are often used in the development of TUIs: RFID, computer vision, and microcontrollers. Then, we compare these technologies using a set of organizing properties. Finally, we describe emerging toolkits and software tools that support the implementation and prototyping of tangible user interfaces, and are based on these basic technologies.

\subsection{RFID}

Radio-Frequency Identification (RFID) is a wireless radio-based technology that enables to sense the presence and identity of a tagged object when it is within the range of a tag reader (an antenna). There are generally two types of RFID tags: active RFID tags, which contain a battery and thus can transmit a signal autonomously; and passive RFID tags, which have no battery and require an external source to initiate signal transmission. In general, RFID tags contain a transponder comprising of an integrated circuit for storing and processing information, and an antenna for receiving and transmitting a signal. Most RFID-based TUIs employ passive inexpensive RFID tags and hence consist of two parts: a tag reader that is affixed to a computational device and a set of tagged objects. The communication between a tag and a reader only occurs when both are proximate. The actual distance varies based on the size of the antenna and that of the RFID tag and the strength of its field. Due to the cost of larger antennas, RFID is usually constrained to short distance detection, with objects required to be placed directly on or swiped past the reader. Some tag readers are capable of detecting multiple tags simultaneously, or writing small amounts of data to individual tags. Other tag readers are read-only 
or only capable of detecting a single tag at a time. When a tag is detected, the tag reader passes an ASCII ID string to the computer. The TUI application can then interpret the ID input string, determine its application context, and provide feedback.

Multiple TUIs are implemented using RFID technology. Examples include a series of prototypes that illustrated the potential of using RFID tags for bridging the Physical and Digital Worlds presented in 1999 by Xerox PARC researchers. These prototypes included augmented books and documents, as well as a photo-cube and a wristwatch [86]. Additional examples include MediaBlocks [236], a TUI that consists of a set of tagged blocks that serve as containers for digital media; Senseboard [120], a TUI for organizing information using a grid that enables the placement of multiple tagged pucks on a white board that is marked with a rectangular grid; and Smart Blocks [79] an educational TUI that computes the volume and surface area of 3D shapes built using tagged blocks and connectors. Martinussen and Arnall [158] discuss the design space for RFID-tagged objects, taking account of the size of tags and the shape of the emitted field.

\subsection{Computer Vision}

In the context of TUIs, computer vision is often used for spatial, interactive surface applications because it is capable of sensing the position of multiple objects on a 2D surface in real time while providing additional information such as orientation, color, size, shape, etc. Computer vision systems can be characterized as being either of the artificial intelligence variety where sophisticated algorithms are used for automatically interpreting a picture, or of the tag variety, where the system tracks specifically defined fiducial markers that are attached to physical objects.

Fiducial marker symbols allow unique marker identities to be distinguished as well as a precise calculation of marker position and angle of rotation on a 2D surface. Since fiducial markers are recognized and tracked by a computer vision algorithm that is optimized for a specific marker design, tag-based systems tend to be more robust, more accurate, and computationally cheaper than systems of the artificial intelligence variety. Thus, tag-based computer vision is often used in the 
development of TUIs. Computer vision TUI systems typically require at least three components: a high-quality camera; a light-weight LCD projector for providing real-time graphical output; and a computer vision software package.

A large variety of TUIs are implemented using tag-based computer vision. Examples include Urp [241], a tangible user interface for urban planning; the reacTable [130], a tangible electro-acoustic musical instrument; Tern [103], a tangible programming language for children, Tangible Interfaces for Structural Molecular Biology [78]; and Tangible User Interfaces for Chemistry Education [68]. The EventTable technique [7] supports event-based rather than object-centric tracking. Using this technique, fiducial markers are cut apart and distributed between objects, so that only when tagged objects are physically connected they form a complete tag that is then detected.

Examples of vision-based TUIs that are not tagged-based include the Designers' Outpost [140], a vision-based TUI for website design that is implemented using an extensive computer vision and image processing algorithm library, the MouseHaus Table [108], and the ColorTable [154], both TUIs for Urban Design that use color and shape to distinguish objects.

Performance and reliability of vision-based systems is susceptible to variations in lighting and motion blur. Using color to identify objects can be relatively robust, but limits object recognition to a small number of high contrast colors. A way to improve the robustness and speed of detection is to paint tokens so they reflect infrared light and to employ a camera filter. This will result in the camera only detecting the painted objects, but reduces the systems' ability to distinguish different objects. This solution has been employed by TUI-related systems such as Build-IT [69].

Several libraries support the development of computer vision-based TUIs. The ARToolkit [131, 133], reacTIVision [130], and Top Codes [103] libraries support the tracking of fiducial markers. Sony researchers developed the CyberCode in 1996 [197], which was preinstalled in some Sony cameras in 1998. Papier-Mâché [139] is a toolkit for building TUIs using computer vision, electronic tags, and barcodes. It introduces a high-level event model for working with computer vision, RFID, and 
bar code, which facilitates technology portability. Using a computer vision library or a toolkit that supports TUI development substantially lowers the threshold of developing computer vision TUIs.

\subsection{Microcontrollers, Sensors, and Actuators}

Microcontrollers act as a gateway between the physical world and the digital world [175]. They are small and inexpensive computers that can be embedded in a physical object or in the physical environment. Microcontrollers receive information from the physical world through sensors, and affect the physical world through actuators. Microcontrollers can be used as stand-alone or they can communicate with a computer. There is a wide variety of sensors and actuators available to be used in embedded systems. Sensor technology can capture a wide range of physical properties including light intensity, reflection, noise level, motion, acceleration, location, proximity, position, touch, altitude, direction, temperature, gas concentration, and radiation. Schmidt and Laerhoven [210] provide a brief but detailed overview of sensor types. Actuators affect the digital world by producing light, sound, motion, or haptic feedback. Microcontrollers may also be connected to RFID readers. Frequently used actuators include LEDs, speakers, motors, and electromagnets.

Many TUI systems are built using embedded microcontrollers. Examples include Posey [252], a poseable hub and strut construction toy; System and Flow Blocks [266], an educational TUI for simulating system dynamics; Senspectra [147] a physical modeling toolkit for sensing and visualization of structural strain; People Pretzel [217], a computationally enhanced play board for group interaction; and Easigami [109], a reconfigurable folded-sheet TUI. These TUI systems use a wide range of microcontrollers and sensors to enable rich and diverse interactions. However, they all provide minimal physical feedback using LEDs while communicating with a computer to provide multimedia digital feedback.

While numerous TUIs are implemented using microcontrollers, relatively few TUIs demonstrate the use of rich physical feedback such as motion, attraction and repulsion. Navigational Blocks [32] is a TUI for navigating and retrieving historical information that illustrates the 
use of haptic physical feedback. The system consists of a set of blocks, each embedded with a microcontroller. Each face in a block represents a query parameter. Thus, each block is capable of sensing its own orientation as well as an adjacent block's orientation. When two blocks are connected, electromagnets in the blocks are used to generate magnetic attraction, or repulsion. This haptic feedback reflects the relationship between the current query parameters. Pico [181] is an interactive surface TUI that uses actuation to move physical tokens upon a surface. Mechanical constraints can then be used to constrain the tokens' movement. The motion of the tokens is generated using an array of 512 electromagnets, a technique that is similar to the technique used in the Actuated Bench [177]. Topobo [196] is a constructive assembly TUI, in which users build structures using connectable pieces. The users can then teach the structure a certain movement by programming active (motorized) pieces using gestures. The active piece records and plays back the physical motion.

While some of the microcontrollers used for developing TUIs require low-level programming skills, several easy-to-use prototyping platforms are currently available for educational purposes as well as for TUI developers from non-technical backgrounds. Such high-level prototyping platforms facilitate iterative development by substantially lowering the threshold for prototyping TUIs. Following, we discuss some examples of such high-level prototyping platforms.

Arduino [12] is an open source physical computing platform based on a simple I/O board and a development environment. Arduino can be used to develop stand-alone interactive devices or can be connected to software running on a computer. The Arduino development environment is a cross-platform Java application that provides a code editor and compiler and is capable of transferring firmware serially to the board. It is based on Processing, a development environment aimed at the electronic arts and visual design communities. The Arduino programming language is related to Wiring, a C-like language. The LilyPad Arduino [26] is a fabric-based microcontroller board designed for wearables and e-textiles. It can be sewn to fabric and similarly mounted power supplies, sensors, and actuators with conductive thread. It is programmed using the Arduino development environment. 
The Handy Board and Handy Cricket are inexpensive and easy-touse microcontrollers aimed mainly at educational and hobbyist purposes. Originally designed as robotics controllers, they were used in the development of multiple TUIs as well as in several TUI laboratory courses including [33, 214]. The Handy Board is programmed in Interactive $\mathrm{C}$, a subset of the $\mathrm{C}$ programming language, the Handy Cricket is programmed using Cricket Logo. The development environments of both microcontrollers are cross-platform. They provide an editor and a compiler, and enable the transfer of firmware to the controllers through a USB connection.

O'Sullivan and Igoe [175] provide an excellent summary of sensors and actuators that can be used with a variety of microcontrollers including Arduino, Handy Board, and Handy Cricket. Lego Mindstorms NXT is a programmable robotics kit that replaces the first-generation Lego Mindstorms kit. The kit has sophisticated capabilities, including servo motor drivers and a variety of sensors such as a sonar range finder and a sound sensor, and can be used for TUI prototyping. Lego has released the firmware for the NXT Intelligent Brick as Open Source. Thus, several SDKs are available for this kit. Finally, the PicoCricket Kit is similar to the Lego Mindstorms robotics kit. However, Lego Mindstorms is designed especially for robotics, while the PicoCricket Kit is designed for artistic creations that include lights, sound, music, and motion. The PicoBoard can be programmed using the Scratch programming Language. While especially attractive for a young audience, it can also be used for rapidly developing functional TUI prototypes.

\subsection{Comparison of Implementation Technologies}

We use the following properties for organizing our comparison of TUI implementation technologies:

(1) Physical properties sensed. What physical properties can be sensed using a particular technology?

(2) Cost. What is the relative cost of the different components comprising a sensing technology?

(3) Performance. Is the system efficient in terms of processing and response times? What factors affect the system's efficiency? 
(4) Aesthetics. To what extent does a sensing technology affect the appearance of an object? Can the user identify which objects or properties are sensed and which are not?

(5) Robustness and reliability. Can the system perform its required functionality for a long period of time? Can the system withstand changing conditions?

(6) Setup and calibration. What is required to get the system in a usable mode?

(7) Scalability. Can the system support an increasing number of objects or users?

(8) Portability. To what extent does a sensing technology compromise the portability of a system?

Table 7.1 describes the comparison of implementation technologies using the above properties.

Table 7.1. Comparison of TUI implementation technologies.

\begin{tabular}{|c|c|c|c|}
\hline Property & RFID & Computer Vision & Microcontrollers \\
\hline $\begin{array}{l}\text { Physical } \\
\text { properties } \\
\text { sensed }\end{array}$ & Identity, presence. & $\begin{array}{l}\text { Identity, presence, } \\
\text { shape, color, } \\
\text { orientation, position, } \\
\text { relative position, and } \\
\text { sequence. }\end{array}$ & $\begin{array}{l}\text { Light intensity, } \\
\text { reflection, motion, } \\
\text { acceleration, location, } \\
\text { proximity, position, } \\
\text { touch, temperature, } \\
\text { gas concentration, } \\
\text { radiation, etc. }\end{array}$ \\
\hline Cost & $\begin{array}{l}\text { Tags are cheap and } \\
\text { abundant. The cost of } \\
\text { readers varies, but is } \\
\text { generally inexpensive } \\
\text { (short distance } \\
\text { readers). }\end{array}$ & $\begin{array}{l}\text { Fiducial tags are } \\
\text { practically free. The } \\
\text { cost of high-quality } \\
\text { cameras continuously } \\
\text { decreases. A } \\
\text { high-resolution } \\
\text { projector is relatively } \\
\text { expensive. }\end{array}$ & $\begin{array}{l}\text { Generally inexpensive. } \\
\text { The cost of sensors } \\
\text { and actuators vary } \\
\text { according to type. }\end{array}$ \\
\hline Performance & $\begin{array}{l}\text { Tags are read in real } \\
\text { time, no latency } \\
\text { associated with } \\
\text { additional processing. }\end{array}$ & $\begin{array}{l}\text { Dependent on image } \\
\text { quality. Tag-specific } \\
\text { algorithms are } \\
\text { typically fast and } \\
\text { accurate. A large } \\
\text { number of tags or } \\
\text { low-quality image take } \\
\text { longer processing. } \\
\text { Motion blur is an } \\
\text { issue when tracking } \\
\text { moving objects. }\end{array}$ & $\begin{array}{l}\text { Generally designed for } \\
\text { high-performance. } \\
\text { Stand-alone systems } \\
\text { typically perform } \\
\text { better than } \\
\text { computer-based } \\
\text { systems. }\end{array}$ \\
\hline
\end{tabular}


Table 7.1. Comparison of TUI implementation technologies.

\begin{tabular}{|c|c|c|c|}
\hline Property & RFID & Computer Vision & Microcontrollers \\
\hline Aesthetics & $\begin{array}{l}\text { Tags can be embedded in } \\
\text { physical objects without } \\
\text { altering their appearance. }\end{array}$ & $\begin{array}{l}\text { Fiducial marker } \\
\text { can be attached to } \\
\text { almost any object } \\
\text { (ideally to its } \\
\text { bottom). }\end{array}$ & $\begin{array}{l}\text { Sensors and actuators } \\
\text { can be embedded } \\
\text { within objects. Wires } \\
\text { may be treated to } \\
\text { have a minimal visual } \\
\text { affect. }\end{array}$ \\
\hline $\begin{array}{l}\text { Robustness and } \\
\text { reliability }\end{array}$ & $\begin{array}{l}\text { Tags do not degrade over } \\
\text { time, impervious to dirt, } \\
\text { but sensitive to moisture } \\
\text { and temperature. Nearby } \\
\text { technology may interfere } \\
\text { with RFID signal. Tags } \\
\text { can only be embedded in } \\
\text { materials opaque to radio } \\
\text { signals. }\end{array}$ & $\begin{array}{l}\text { Tag-based systems } \\
\text { are relatively } \\
\text { robust and reliable. } \\
\text { However, tags can } \\
\text { degrade over time. } \\
\text { Detection only } \\
\text { within line of sight. }\end{array}$ & $\begin{array}{l}\text { Typically designed for } \\
\text { robustness and } \\
\text { reliability. Batteries } \\
\text { need to be charged. } \\
\text { The robustness and } \\
\text { reliability of sensors } \\
\text { and actuators vary. } \\
\text { Wiring may need to } \\
\text { be checked. }\end{array}$ \\
\hline $\begin{array}{l}\text { Setup and } \\
\text { Calibration }\end{array}$ & $\begin{array}{l}\text { Minimal. No line of sight } \\
\text { or contact is needed } \\
\text { between tags and reader. } \\
\text { The application must } \\
\text { maintain a database that } \\
\text { associates ID with desired } \\
\text { functionality. }\end{array}$ & $\begin{array}{l}\text { Address a variety } \\
\text { of factors including } \\
\text { occlusion, lighting } \\
\text { conditions, lens } \\
\text { setting, and } \\
\text { projector } \\
\text { calibration. }\end{array}$ & $\begin{array}{l}\text { Connect } \\
\text { microcontroller to } \\
\text { computer; wire } \\
\text { sensors and actuators; } \\
\text { embed hardware in } \\
\text { interaction objects; } \\
\text { fabricate tailored } \\
\text { interaction objects to } \\
\text { encase hardware. }\end{array}$ \\
\hline Scalability & $\begin{array}{l}\text { The number of } \\
\text { simultaneously detected } \\
\text { tags is limited by the } \\
\text { reader. No practical } \\
\text { limitation on the number } \\
\text { of tagged objects. }\end{array}$ & $\begin{array}{l}\text { The maximal } \\
\text { number of tracked } \\
\text { tagged objects } \\
\text { depends on the tag } \\
\text { design (typically a } \\
\text { large number). }\end{array}$ & $\begin{array}{l}\text { Typically constrained } \\
\text { by the number of I/O } \\
\text { ports available on a } \\
\text { microcontroller. }\end{array}$ \\
\hline
\end{tabular}

\subsection{Tool Support for Tangible Interaction}

Several toolkits and software libraries have emerged to support the implementation of functional TUI prototypes. This section outlines some existing tools for tangible interfaces, as well as tools that support reality-based interaction styles. We selected to discuss tools and libraries that contribute a technical solution as well as a novel approach for developing TUIs. Additionally, tools, libraries, and prototyping platforms are discussed above within the sections on specific implementation technologies. 
The commercially available Phidgets (http://www.phidgets.com/) provides a set of "plug and play" USB-attached devices (e.g., I/O boards, sensors, and actuators) that are analogous to widgets in graphical user interfaces [82, 81]. For example, Phidets allows any analog sensor to be plugged into its board, as long as it modulates a 5 -V signal. Similarly, any on/off switch and other digital I/O devices can be plugged to the board and controlled by a binary value. Phidgets are aimed to support software developers in the implementation of mechatronic TUI prototypes composed of wired sensors and actuators. Such TUIs are capable of both physical input and physical output. The main advantage of Phidgets is that they are centrally controlled through a conventional computer rather than through a standard microprocessor. Thus, the integration of digital capabilities such as networking, multimedia, and device interoperation becomes easier. Another advantage is ease of programming and debugging which are significantly more difficult to do when one compiles and downloads a program to a microprocessor. The Phidgets API supports application development in a variety of development environments. Shared Phidgets [155] is an extension of Phidgets that supports rapid prototyping of distributed physical interfaces. Shared Phidgets automatically discovers devices connected to a myriad of different computers and allows users to centrally control a collection of remote interoperable devices by creating abstract devices and simulating device capabilities.

iStuff [11] is similar to Phidgets in concept but uses Java to control a set of light-weight wireless physical devices. iStuff is aimed at enabling interaction designers to rapidly prototype applications for an environment called the iRoom. Through an intermediary software called the Patch Panel, interaction designers can define high-level events and dynamically map them to input and output events. iStuff Mobile [10] is built on top of the iStuff framework to enable the rapid prototyping of sensor-based interaction with mobile phones. The European Smart-Its project developed another toolkit for physical prototyping, based on self-contained, stick-on computers that attach to everyday objects [75]. Each Smart-It can communicate with other Smart-Its or IT-devices and can sense data about its surroundings. 
Exemplar [87] is a toolkit for authoring sensor-based interactions that similarly to Phidgets leverages central control through a conventional computer to allow TUI developers to link sensor input data to application logic by generating discrete events from sensor input. Exemplar provides low-level support for integrating hardware into a TUI and higher-level abstractions for sensor input. With Exemplar, a designer demonstrates a sensor-based interaction to the system (e.g. shaking an accelerometer). The system then graphically displays the resulting sensor signals. The designer can iteratively refine the recognized action and, when satisfied, use the sensing pattern in prototyping or programming applications. Exemplar is implemented as an Eclipse plug-in and supports the development of Java applications. Both Exemplar [87] and Shared Phidgets [155] go beyond a single processor type.

We have already discussed Arduino [12] in the previous section on implementation technologies. It is a toolkit consisting of the Arduino board and programming environment. Different from Phidgets and iStuff, which entail specifically built sensors and actuators that are easily plugged together and are centrally controlled through a conventional computer, Arduino interfaces with standard electronics parts. Arduino thus does not "black-box" the electronics, but requires physical wiring, circuit building, and soldering.

The VoodooIO system is similar to Phidgets in providing a range of physical controls but emphasizes the malleability of physical interfaces [247]. VoodooIO uses a substrate material on which controls can be dynamically added, arranged, manipulated, and removed (Figure 7.1). This substrate material effectively serves as a network bus to which controls can be connected effortlessly, wirelessly, and rapidly as well as energy supply. Integration of VoodooIO functionality into interactive applications is supported in a number of programming environments. While VoodooIO is aimed at software developers, VoodooFlash [226] is a design tool aimed at interaction designers. It integrates Flash with VoodooIO and is based on the Flash concept of a stage on which interactive components are arranged in the process of designing an interface. Alongside the graphical stages in Flash, VoodooFlash provides a physical stage on which designers can arrange physical controls. The graphical and physical stages are closely coupled. The VoodooFlash 


\section{Implementation Technologies}

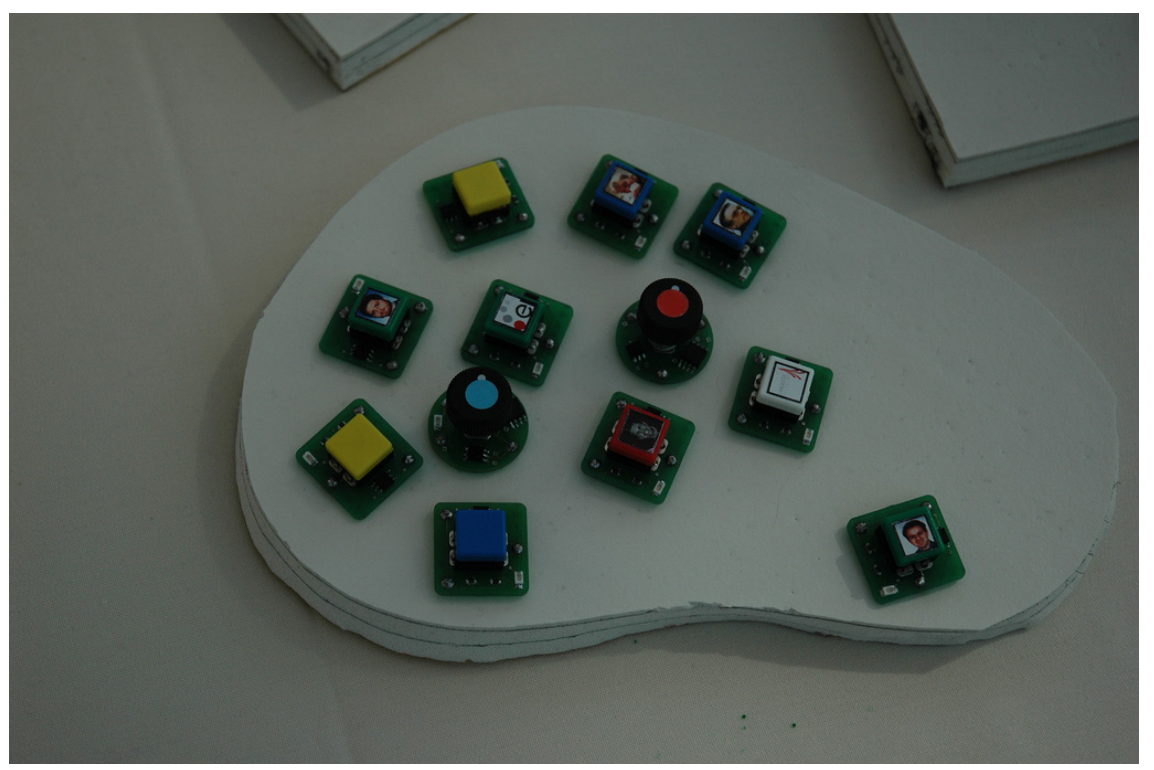

Fig. 7.1 The VoodooIO controls [247] can be dynamically added to and arranged upon a substrate material that acts as a network bus (photo courtesy of M. Kranz, TU München).

system handles the communication, parsing, and event dispatching between Flash and VoodooIO.

Papier-Mâché [139] provides higher-level API support for acquiring and abstracting TUI input from computer vision, RFID and barcodes as well as for easily porting an interface from one technology to another. Through technology-independent input abstractions, PapierMâché enables software developers to rapidly develop functional TUI prototypes as well as to retarget an application to a different input technology with minimal code changes. Using computer vision, RFID, and barcode, Papier-Mâché supports the development of TUIs that track passive, untethered objects such as paper notes and documents. The toolkit handles the discovery of and communication with input devices as well as the generation of high-level events from low-level input events. To facilitate debugging, Papier-Mâché provides a monitoring window that display the current input objects and behaviors being created or invoked. The monitoring window also provides Wizard of $\mathrm{Oz}(\mathrm{WOz})$ generation and removal of input. WOz control is useful 
for simulating hardware when it is not available, and for reproducing scenarios during development and debugging. Similar to Exemplar [87], Papier-Mâché is implemented as an Eclipse plug-in and supports the development of Java applications.

Another physical toolkit currently under development is the Littlebits (www.littlebits.cc) that consists of discrete electronic components, pre-assembled on tiny circuit boards, which snap together through tiny magnets. Several toolkits support the development of GUIs with physical controls and handheld devices, such as d.tools [88], the Calder toolkit [8], and the IE Unit [77].

Only some of the listed physical toolkits are available commercially, and readers should investigate prices and availability in their home country. Toolkits entailing electronics by their very nature, even if they are open source, cannot be made available for free. Both computer vision software toolkits that we now describe are available for free and only require a webcam in order to develop prototypes.

ARToolKit $[131,133]$ is a computer vision marker tracking library that allows software developers to rapidly develop augmented reality applications. In addition to tracking the 3D position and orientation of square markers, it enables to overlay virtual imagery on a real physical object tagged with a marker. To do this, it calculates the real camera position with respect to a marker, and then positions a virtual camera at the same point. Three-dimensional computer graphics models can then be drawn to exactly overlay the real marker. While originally aimed at augmented reality application, the ARToolkit is often used for developing TUIs (for an experience report see e.g., [107] and Figure 7.2). The ARToolkit is usually used in combination with a Tracker library. Having been developed to support the development of Augmented Reality applications, the ARToolkit provides 3D information (size of marker, angle of view), but employs a format tailored to 3D VR imaging for its output, thus, rendering interpretation of the $3 \mathrm{D}$ information for other purposes somewhat difficult.

Finally, the reacTIVision framework [130] is a cross-platform computer-vision framework primarily designed for the construction of tangible multi-touch surfaces. It enables fast and robust tracking of fiducial markers attached onto physical objects, as well as multi-touch 


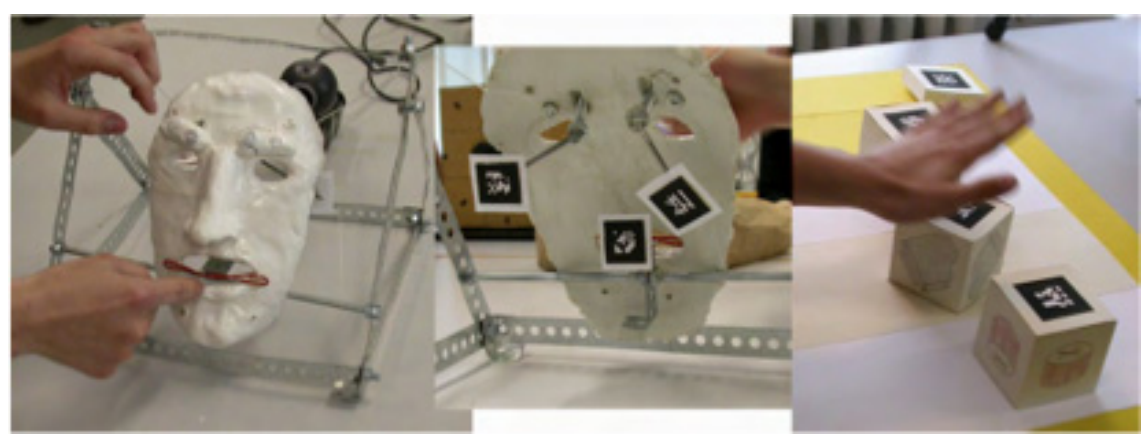

Fig. 7.2 TUI prototypes built with the ARToolkit: Left and middle: changing the facial expression of the mask moves the markers in the back, these changes are computationally interpreted to instruct a music application to select a different style of music; Right: The position of blocks on the $x$ and $y$ axis is tracked and represents different musical instruments [107].

finger tracking. The central component of the framework is a standalone application for fast and robust tracking of fiducial markers in a real-time video stream. The underlying transport protocol, TUIO, supports the efficient and reliable transmission of object states via a local or wide area network. It has become a common protocol and API for tangible multi-touch surfaces. The reacTIVision toolkit (at the time of writing this monograph) only implements the TUIO 2D protocol; it does not provide 3D information. TUIO is more general and also provides a $2.5 \mathrm{D}$ (distance of markers to camera) and $3 \mathrm{D}$ protocol. There are various TUIO tracker implementations, including for the Wiimote (see http://www.tuio.org/?software).

In the future ReacTIVision may be extended to describe the space above the surface. The major difference between reacTIVision and other toolkits is its distributed architecture, separating the tracker from the actual application. TUIO provides an abstraction layer for tracking, and thus allows the transmission of the surface data to clients. This approach facilitates the development of TUIO clients in various programming languages.

The major advantage of the tools discussed above is that they lower the threshold for implementing fully functional TUI prototypes by hiding and handling low-level details and events. Hence, they significantly reduce the duration of each design/implementation/test cycle. 
However, as each of these tools provides support for specific technology and hardware components, each time a TUI is prototyped using different technology and hardware (it is common for new prototypes to be developed several times throughout a development process) a TUI developer is required to learn a new toolkit or software library and rewrite code. Furthermore, toolkits tend to codify common interaction techniques into a standard set of widgets, thereby excluding other interaction styles [107]. While the effort to codify existing interaction techniques has begun, the search for new interaction techniques and technological solutions still continues. Thus, software tools that can be easily extended to support new interaction techniques and technologies are needed. Finally, although toolkit programming substantially reduces the time and effort required for software developers to build fully functional TUI prototypes, it falls short of providing a comprehensive set of abstractions for specifying, discussing, and programming tangible interaction within an interdisciplinary development team. 


\subsection{Design and Implementation}

While tangible interaction shows promise to enhance computermediated support for a variety of application domains including learning, problem solving, and entertainment, TUIs are currently considered difficult to design and build. In addition to the challenges associated with designing and building traditional user interfaces [166], TUI developers face several conceptual, methodological, and technical difficulties. Among others, these challenges include: the lack of appropriate interaction abstractions, the shortcomings of current user interface software tools to address continuous and parallel interactions, as well as the need to cross disciplinary boundaries in order to interlink the physical and digital worlds. Shaer and Jacob investigated the development process of TUIs and provided a detailed discussion of TUI development challenges in [215].

General design approaches for TUIs range from designer-led to user- centered and problem-oriented. Baskinger and Gross [14] claim that TUIs directly link interaction designers with product development. They highlight the need for new design processes that encourage experimentation while integrating code, product form, behavior, 
information, and interaction. A few research teams have successfully applied an iterative user-centered design approach in which the development of prototypes and prototype evaluation in the field inform redesign [243]. Fernaeus and Tholander [57] conducted extensive video interaction analysis of pupils working with different low-fidelity prototypes for a tangible programming tool before building the final system. Maquil et al. [154] applied an iterative process of design-evaluationfeedback-redesign in the design of a TUI for urban planning, and evaluated a series of prototypes by deploying them in user workshops in the context of real urban planning projects. However, only few principled approaches for TUI design were proposed. Edge and Blackwell [51] propose an "analytic design process" which can be viewed as a rational, progressive derivation of a design from a design context. This process consists of four stages, starting with contextual analysis to identify activities that could benefit from TUI support, activity analysis to describe the TUI properties for supporting these activities, a mapping analysis that generates the physical-digital mappings of a TUI with these properties, and finally a meaning analysis that provides a meaning to these mappings.

Following, we describe some of the methods and techniques used in the development process of TUIs. We focus on the adaptation of traditional design methods to TUI development and on emerging methods dedicated to TUI design.

\subsubsection{Ideation and Sketching}

As common in other design disciplines, sketches dominate the early ideation stages of TUI development [30]. They are used for experimenting with high-level ideas and with aspects such as tangible representations, form factors, user experience, and possible relationships between physical interaction objects. However, drawn sketches are not always sufficient to explore TUI design ideas. Simple physical form prototypes or mock-ups thus play a larger role in TUI design than in traditional HCI. Product designers sometimes refer to physical models as (3D or physical) sketches. The shared characteristic of sketches, be they free hand sketches, storyboards, or lo-fi prototypes and mock-ups, 
is that they are quick, timely, inexpensive, plentiful, and disposable artifacts [30]. Most of these techniques support the representation of and experimentation with design ideas.

As a more general technique, Hornecker [104] presents a card brainstorming exercise that transforms the more conceptual tangible interaction framework [105] into a tool for creative ideation that depicts aspects of the framework on individual playing cards which are used in a brainstorming exercise.

Storyboarding is a common technique in the design of interactive systems for demonstrating system behavior and contexts of use [233]. However, it is less effective for describing interaction with TUIs, as continuous and parallel multi-user interactions are difficult to depict. Rather, storyboards are often used to describe a TUI's larger context of use - illustrating an envisioned use scenario and depicting its physical surroundings.

Physical sketches (prototypes and mock-ups) enable the designer to explore the interaction by enacting scenarios. In TUI design, low fidelity prototypes and mock-ups are often rapidly built using simple construction materials (e.g., blue foam or modeling clay) in order to examine aspects related to the form and function of a TUI such as physical handling, as well as to communicate alternative designs to users or within an interdisciplinary development team. Blackwell et al. [20] interpret low-fidelity prototypes of TUIs as "solid diagrams" and suggest that applying correspondence analysis to solid diagrams can provide both device-centric and user-centric understanding of a TUI design. However, doing so requires some experience and guidance.

As actuated TUIs have begun to emerge, there is a need for design methods that support investigating tangible user interfaces that transform in time and space. Parkes and Ishii [178] propose a methodology for designing TUIs with kinetic behaviors. Their approach defines variables, combinations, and possibilities of a motion design language. This Kinetic Sketchup system provides architects and product designers with a system of mechanical modules that can be physically programmed through gestures to exhibit a particular kinetic behavior. Parkes and Ishii deconstruct the kinetic prototyping space into material, mechanical, and behavioral properties. A pictorial notation enables to specify 
each module by constructing a "motion phrase". While the Kinetic Sketchup methodology enables designers to explore transformation through motion, this prototyping process is separate from the design of the underlying software structure. Within Product Design there is an emerging practice of " $4 \mathrm{D}$ sketching" to explore product movement [56]. This interprets time as the fourth dimension, focusing on the relation of form and movement. These new design approaches experiment with manipulating video (see e.g., [29]), with motion scratchbooks, enactment by the designers themselves (imitating product movement), and with animation techniques from puppetry [259, 260]. Buur et al. [29] started to investigate how to support rich and skilled movement in tangible interaction. Their design approach seeks inspiration from users' existing practices, aiming to retain the quality of their movement, by extracting qualities of movements from video and designing an interface that regenerates these types of movements [122].

\subsubsection{Building Functional Prototypes}

Functional TUI prototypes are used for investigating the function of a TUI and evaluating a design concept with users. Several toolkits have emerged to support implementation. Examples include: Phidgets [82], iStuff [11], Papier-Mâché [139], Exemplar [87], and the Arduino platform. We discuss toolkits and frameworks for developing functional TUI prototypes in detail in Section 6.

The major advantage of such toolkits is that they lower the threshold for implementing fully functional TUI prototypes by hiding and handling low-level details and events, hence, significantly reducing the duration of each design/implementation/test cycle. Furthermore, they enable designers to experiment with interactive behaviors during early design stages. However, at different stages of design different toolkits might be more suited as they each provide support for different hardware components. This requires a TUI developer to learn a new toolkit and rewrite the TUI code. Furthermore, toolkits tend to codify common interaction techniques into a standard set of widgets, but as the effort to codify existing interaction techniques has begun, the search for new interaction techniques and technological solutions continues. 
Finally, toolkit programming falls short of providing a comprehensive set of abstractions for specifying, discussing, and programming tangible interaction within an interdisciplinary development team.

\subsubsection{Semi-formal Specifications}

Shaer and Jacob [215] proposed a design methodology for TUIs that is based on the User Interface Description Language (UIDL) approach and on UIMS research [172]. It is aimed at addressing challenges of TUI development including the lack of appropriate interaction abstractions, the definition and implementation of continuous and parallel interactions, and the excessive effort required for porting a TUI from one implementation technology to another. Applying this methodology, TUI developers would specify the structure and behavior of a TUI using high-level constructs, which abstract away implementation details. These specifications can then be automatically or semi-automatically converted into different concrete TUI implementations by a Tangible User Interface Management System (TUIMS). In addition, such specifications could serve as a common ground for investigating both design and implementation concerns by TUI developers from different backgrounds.

To support this approach, Shaer and Jacob introduced Tangible User Interface Modeling Language (TUIML), a visual high-level user interface description language for TUIs that is aimed at providing TUI developers from different disciplinary backgrounds means for specifying, discussing, and iteratively programming tangible interaction. TUIML consists of a visual specification technique based on Statecharts [85] and Petri Nets [187], and an XML-compliant language. Shaer and Jacob also presented a top-level architecture and a proof-of-concept prototype of a TUIMS that semi-automatically converts TUIML specifications into concrete TUI implementations. It is important to note that TUIML was mostly designed to specify datacentered TUIs [105], which are systems that use spatially configurable solid physical artifacts as representations and controls for digital information [238]. Currently, TUIML does not support tangible interaction techniques such as expressive gestures and choreographed actions. 


\subsection{Evaluation}

The evaluation methods used to study tangible user interfaces are similar to those used within HCI. So far no evaluation methods specific to TUIs have been developed. Given the novelty of the field and its initial emphasis on proof-of-concept prototypes, this is perhaps little surprising. The most frequent types of evaluations are comparative studies, often in the form of quantitative empirical lab studies, heuristic evaluations, and qualitative observation studies, often based on video analysis and sometimes conducted in the field.

\subsubsection{Comparative Studies}

Comparative studies attempt to quantify the costs and benefits of tangible interaction, compared to other interaction styles, typically a graphical user interface, or to compare different variants of a tangible interface. They vary from empirical lab studies to studies conducted in the wild. Traditionally, comparative studies focus on objective quantitative measurements such as task completion time, error rate, and memorization time. However, recently several studies attempted to quantify more high-level interaction qualities such as enjoyment, engagement and legibility of actions. Subjective data can be collected through observation and questionnaires. We briefly give two examples of controlled comparative experiments that focus on quantitative measurement of traditional performance indicators. In the Senseboard study [120] each subject performed a scheduling task under four different conditions, measuring speed of performance. To evaluate and compare four alternative interactions techniques for the GeoTUI interface, Couture et al. [42] conducted a within-subjects study at the workplace of geophysicists using the task of selecting cutting planes on a geographical map, measuring completion time and collecting questionnaire data.

Several recent studies take a different evaluation approach by taking place in the field. Rather than focusing on traditional performance measurements, these studies measure higher-level interaction qualities such as legibility of actions, user engagement, and collaboration. Unfortunately, field studies of TUIs are still rather rare. The following examples illustrate a range of feasible approaches. 
Pedersen and Hornbaek [183] provide an interesting example of evaluation through live performance. MixiTUI is a tangible sequencer for electronic musicians. To assess its performative value, an audience of more than 100 participants answered a questionnaire after a concert in which two songs were played using traditional laptop instruments and two using the MixiTUI interface. Another field evaluation with a large number of participants was conducted by Parmar et al. [179] in Western India. A new TUI for a health information system for rural women was evaluated in group sessions with 175 women that had previously used the same information system using an iconic keyboard interface. The study goal was to measure changes in social interaction. The sessions were recorded on tape. Participants were also asked to rate both systems in terms of engagement and social interaction generated. Finally, Horn et al. [102] conducted a between-subjects study to compare the effectiveness of a tangible and a graphical programming interface for the Robot Park exhibit at the Boston Museum of Science. The two interfaces were as similar as possible. Each variant was exhibited for a week, and video and computer logs were collected. The analysis investigated for example the percentage of visitors interacting with the exhibit, how long they interacted, how many people in each visitor group interacted, and how complex the programs developed were in each condition.

\subsubsection{Ethnographic Observation and Video Analysis}

Ethnographic-style observation and interaction analysis approaches developed in the work studies tradition $[90,126]$ have been very influential in the field of HCI [220]. Interaction analysis of video is well suited to the study of TUI use, because it provides an integrated approach for investigating verbal and nonverbal behaviors, and focuses on the role of physical objects within a system. Studies using these methods of qualitative observation tend to remain open to new aspects, develop analysis criteria iteratively based on the observed data, and are only coarsely guided by a loosely phrased hypothesis. For field studies with smaller numbers of participants, qualitative observation tends to be the method of choice next to interviews. Qualitative analysis is also useful 
to develop a hypothesis that is then tested in a specifically designed experimental study.

The depth of analysis in observational studies of TUIs varies greatly, ranging from typical user or usability studies (where researchers take notes of frequent problems and typical behaviors) to transcriptions of videos with a detailed moment-to-moment interaction analysis. We now briefly describe a number of studies to show this diversity. One of the earliest evaluation studies of a TUI, the AlgoBlocks programming language, was presented by Suzuki and Kato [231]. Analysis focused on how children utilized body movement and positioning to coordinate their activity and how the TUI engendered this. Tangicam, a tangible camera for children [144] was evaluated by asking young children at a science fair to explain the toy to another child. Zuckerman et al. [266] interviewed children while they were working to complete a set of tasks using the FlowBlocks, probing their understanding of the tasks and the system. Ryokai et al. [205] conducted a small field study of I/O Brush, an augmented painting tool for children, setting the system up in a corner of a kindergarten, observing children's interactions with the system, and studying the results of their creative activity. Trial studies (see [267] for a good example), in which a system is used in the field, but only for a limited time and/or by a select small group, are very useful for discovering usability issues, initial attractiveness of the system, and what features people quickly discover. Disadvantages of trial studies are that users might rate the system high because of its novelty, and that effects of long-term adaption and learning cannot be investigated. In user-centered design, evaluation methods are often employed to inform the iterative design process. Some research teams work extensively with users through approaches related to action research and participatory design and employ video analysis to inform an iterative design process (cf. $[57,154])$. 
9

\section{Strengths and Limitations of Tangible User Interfaces}

The central tenet for human-computer interaction is to support users in accomplishing a set of tasks. Different tasks are better supported by different interaction styles. Thus, an understanding of the strengths and limitations of a particular interaction style is essential for determining whether it is adequate for supporting certain tasks. From a problem-oriented design viewpoint tangibility as design objective might not always be the right solution. Yet from a research standpoint, there is often a value in exploring what tangibility can get us, and discovering different approaches [106]. Such explorations provide us with an increasingly clearer picture of the strengths and limitations of TUIs. Good design aims to bring out the strengths and to alleviate weaknesses, for example by building on the strengths of tangible elements and drawing on the strengths of other related areas for accomplishing qualities that cannot be accomplished by a TUI alone. Conversely, integration of tangible elements can alleviate interaction problems of non-tangible systems (such as multi-touch tables, cf. [135]).

Following, we discuss some of the strengths and limitations of TUIs. However, it is important to note that TUI research is still in its infancy and hence our understanding of the implications of TUIs requires further investigation. 


\subsection{Strengths}

\subsubsection{Collaboration}

From the very beginning, an underlying aim of many TUI systems has been to foster a dialog between domain expert (e.g., architects) and concerned parties (e.g., future inhabitants of buildings), and to support collaborative learning (cf. [3, 71, 241, 230]).

Hornecker and Buur [105] list three factors that support face-toface collaboration. Familiarity and affordances known from everyday interaction with the real world lower the threshold for engaging with a system and thus increase the likelihood of users to actively contribute. Tangibles have been shown to have an inviting quality [102] compared to mouse-screen-based interfaces, yielding higher numbers of visitors using them in a museum context, especially more children and females. Multiple access points ensure that there is no "bottleneck for interaction", allowing for simultaneous interaction and easing participation. Furthermore, manual interaction with objects is observable and has enhanced legibility due to the visibility of the physical objects (cf. [138]). This supports group awareness and coordination. Through careful design of the physical setup, an interface can provide "embodied facilitation", subtly constraining and guiding users' behaviors. For example, Jordà's ReacTable [124] was deliberately given a circular shape so as to foster sharing and to provide equal access for a varied number of users. Providing a single set of cards [2] can also encourage sharing. Furthermore, tangible objects can be handed over and shared more easily than graphics (cf. [78]), thus tangible objects foster shared discussion.

Jordà [124] argues that the ability for simultaneous action and its visibility to collaborators makes a tangible tabletop interface superior over graphical interfaces for sharing control of real-time data such as in music performance. Tangible artifacts can be understood as resources for shared activity $[57,58,59]$. This includes 'offline' activities where a group might be planning its next actions laying out tangible objects to represent a plan of action. In her overview of the role of tangible interfaces for learning, Antle [6] describes tangibles as having "both the space and the affordances for multiple users", creating "space for 
friends". Tangible input might also very subtly support collaboration and social interaction. In the Linguabytes project, the TUI that is used for supporting speech therapy sessions for disabled toddlers slows down the interaction, creating more time for adult-child interaction [93, 94].

\subsubsection{Situatedness}

Tangible interfaces can be interpreted as a specific implementation of the original notion of Ubiquitous Computing [253], which aimed at allowing users to remain situated in the real world, and retaining the primacy of the physical world. Yet, while embedded in context, the design goal for tangible interfaces is not the invisibility of the interface, but rather the physicality of the interface.

As discussed by Dourish [50], one of the main strengths of tangible interfaces is that they can inhabit the same world as we, and are situated in our lifeworld. Similarly, Hornecker and Buur [105] argue that Tangible Interaction is embedded in real space and thereby always situated in concrete places. Tangible interfaces, by not just residing on a screen, are just as much a part of the physical environment as architectural elements or physical appliances and products. This situated nature of TUIs makes them very powerful as UbiComp devices. Situatedness furthermore implies that the meaning of tangible interaction devices can change depending on the context in which they are placed, and reversely, they can alter the meaning of the location.

Understanding and designing for interaction-in-context is one of the elements of what Fernaeus et al. [58] have termed the 'practice turn' in tangible interaction. It concerns research that emphasizes how tangible interaction can blend into everyday activities and integrate with qualities of the interaction setting. This implies thinking about the interactions around the system, and how people interact with each other even when this activity is not directly directed at the interface. Physical interaction will often result in many manipulations of interface elements being performed 'offline', directed at the social and physical setting. Tangible interfaces, often consisting of multiple tangible objects that can be carried about and rearranged in space, support this distribution of activity around the actual interface. 


\subsubsection{Tangible Thinking}

Our physical body and the physical objects with which we interact play a central role in shaping our understanding of the world [138, 234]. Infants develop their spatial cognitive skills through locomotive experience. Children learn abstract concepts through bodily engagement with tangible manipulatives [199]. Professionals such as designers, architects, and engineers often use physical artifacts to reason about complex problems [57]. One of the strengths of TUIs compared to traditional user interfaces is that they leverage this connection of body and cognition by facilitating tangible thinking - thinking through bodily actions, physical manipulation, and tangible representations.

Klemmer et al. [138] provide a good overview of tangible thinking that includes perspectives of educational theory, gesture research, and cognitive science. They highlight five aspects of tangible thinking that relate to theories of external representation [207] and distributed cognition [98], and to the study of gesture. Turkle introduces the concept of evocative objects [234], day-to-day objects that serve as emotional and intellectual companions, anchor memories, sustain relationships, and provoke new ideas. Through a collection of personal essays she demonstrates the role of everyday objects in facilitating emotional and cognitive development. Following, we briefly discuss three aspects of tangible thinking: gesture, epistemic action, and tangible representation. A detailed discussion of these topics can be found in Section 6.3, [138], and [234].

\subsubsection{Gesture}

While gestures are typically considered as means of communication, multiple studies have illustrated that gesturing plays an important role in lightening cognitive load for both adults and children [5], and in conceptually planning speech production [80]. By providing users with multiple access points to the system and maintaining their physical mobility (as hands need not be confined to the keyboard and mouse), TUIs enable users to take advantage of thinking and communicating through unconstrained gestures while interacting with a system. 
Some TUIs (as well as other emerging interaction styles) utilize gesture as input modality either in the form of a symbolic gesture language or as imitation of real-world daily actions. TUIs that employ gesture as interaction modality take advantage of users' kinesthetic memory [213], the ability to sense, store and recall muscular effort, body position and movement to build skill. Kirk et al. [135] reason that the kinesthetic memory of moving a tangible object can increase the awareness of performed actions, helping to reduce the risk of mode errors. Furthermore, because many daily actions such as driving, operating tools and engaging in athletic activities are skillful body-centric behaviors, TUIs that utilize gestures which imitate such bodily actions leverage body-centric experiential cognition [169], the kind of thought that enables to generate an immediate response without apparent effort but requires years of experience and training. Tangibles furthermore support 3D manipulation in ways that surface-based computing cannot (cf. [135]).

\subsubsection{Epistemic Actions and Thinking Props}

Various studies have demonstrated that physical artifacts support cognition by serving as "thinking props" and external memory (see section 6.3). In a seminal paper, Kirsh and Maglio [137] make a distinction between pragmatic actions that have functional consequences and hence contribute toward accomplishing a goal, and epistemic actions that do not have functional consequences but rather change the nature of the mental task. Epistemic actions help to explore options, keep track of previous paths taken, and support memory. Actions such as pointing at objects, changing their arrangement, turning them, occluding them, annotating and counting, may serve as epistemic actions that decrease the mental workload of a task by drawing upon resources that are external to the mind. By facilitating a relatively free form interaction with physical objects and allowing 'out of band interaction' that is not computationally interpreted, TUIs tend to make epistemic actions easier than traditional user interfaces. They support a wide range of actions, utilize a wide range of physical objects, and allow for differentiated actions. Several studies provide evidence for the ways in which tangible interaction supports epistemic actions and cognition 
$[41,57,134,180]$. A more detailed discussion of epistemic actions, external, and distributed cognition can be found in Section 6.3.

\subsubsection{Tangible Representation}

In a widely known study, Zhang and Norman [262] demonstrated that the representation of a task can radically affect reasoning abilities and performance. Studying alternative representations (i.e., isomorphs) of the games of Tic-Tac-Toe and the Towers of Hanoi, they found that an increase in the amount of externally represented information yielded improvement in solution times, solution rates, and error rates. Based on these results, Zhang [261] went on to conclude that external representations are intrinsic components of many cognitive tasks as they guide, constrain, and even determine cognitive behavior.

There are many different ways in which TUIs employ physical objects as external representations of digital information: some application domains such as architecture, urban planning, and chemistry have inherent geometrical or topological representations that can be directly employed in a TUI; other domains such as economics, biology, and music do not have inherent physical representations but have representational conventions that may lend themselves to spatial representations. Finally, domains such as information navigation or media authoring do not have inherent or conventional spatial representations but may be tangibly represented using symbolic or metaphoric mapping. In all cases, interaction with physical representations leverages peoples knowledge and skills of interaction with the real non-digital world such as naïve physics, body awareness and skills, social awareness and skills, and environment awareness and skills (cf. section 3.3, [119]).

Finally, Ullmer et al. [239] proposed an approach for tangible interaction that centers on a hierarchical relationship between two kinds of physical elements: Tokens and Constraints (see section $5.3-$ Classifications of TUIs). In their token+constraint approach, tokens are physical interaction objects that can be placed within or removed from compatible constraints. Compatibility is expressed through the physical shape of the tokens and constraints, where incompatible elements 
do not mechanically engage. This approach uses physical properties to create a physical syntax that perceptually encodes interaction syntax. Such physical syntax not only decreases the need for explicit rules [262] but also supports perceptual inference, direct reading by human perception that does not require explicit logical deduction $[114,207]$.

\subsubsection{Space-Multiplexing and Directness of Interaction}

"With space-multiplex input each function is controlled with a dedicated transducer, each occupying its own space. Each transducer can be accessible independently but also simultaneously." "In contrast, time-multiplex input uses one device to control different functions at different points in time." [65]

In tangible interfaces that employ multiple interaction objects, input is space-multiplexed. Different physical objects represent different functions or different data entities. This enables the system designer to take advantage of shape, size, and position of the physical controller to increase functionality and decrease complexity of interaction. In addition, it allows for more persistent mappings, compared to a traditional time-multiplexed GUI, where each mouse click might result in a different function being evoked or a different object selected.

Without spatial multiplexing, input objects are generic and thus need to have abstract shape and appearance. With static mappings and multiple input objects, tangible input elements (tokens) can be expressive and may furthermore provide affordances [168] specific to the functionality they give access to. Spatial multiplexing thus is an enabler of strong-specificness (see next section).

Multiple specific objects support parallel actions. In contrast, with a GUI a user has to sequentially perform one action after the other. Parallel actions potentially speed up the task, and support eyes-free interaction, as the remaining objects in the workspace can still guide the hand. Fitzmaurice [65], further notes that spatially multiplexed objects may allow us to "tap into our spatial memory (or "muscle memory')". Furthermore, in a traditional GUI there can only be one active selection at a time and any new selection undoes a prior one. A TUI 
can eliminate many of the redundant selection actions (choose function, do action, choose next function). User studies have demonstrated that spatial multiplexing is superior to time-multiplexed interaction in performance [65] and lowers the cost of 'acquiring' input devices.

Beaudouin-Lafon [15] provides a structured explanation of how, by involving less steps of mediation, TUIs can result in more direct interaction. First, he distinguishes input devices (e.g., mouse), interaction instruments (e.g., a slider), and interaction objects (the domain entity worked upon, e.g., text). He then proposes to measure the directness of manipulation along the relations between these three entities (spatial and temporal distances between instrument and interaction object, differences in the degrees of freedom between input device and interaction instrument, and similarity between manual input action and result on the domain entity). For a TUI, input devices can have persistent mappings and are simultaneously accessible. This means that interaction instruments are instantiated through a dedicated physical input device. Furthermore, interaction objects might be represented in physical form and thus serve as their own input device. In BeaudoinLafon's terms, TUIs thus reduce indirectness and improve integration and compatibility.

\subsubsection{Strong-Specificness Enables Iconicity and Affordances}

Employing multiple input objects (space-multiplexing) means that these do not need to be abstract and generic but can be strong-specific [65], dedicated in form and appearance to a particular function or digital data (cf. Figure 9.1). In a TUI, tokens can have persistent mappings. Thus, an object's appearance can directly indicate its meaning or function as well as how to interact with it, by making use of physical affordances. Furthermore, strongly specific objects can constrain manipulation to allow (or invite) only those actions that have sensible results [168]. Strong specificness can thus improve the mapping of actions to effects (cf. [15, 254]). Tangible interfaces, through their very nature of being physical and thus less malleable and less mutable than a purely digitally computer-controlled representation, tend to be strong-specific. This is both a strength and a weakness. 

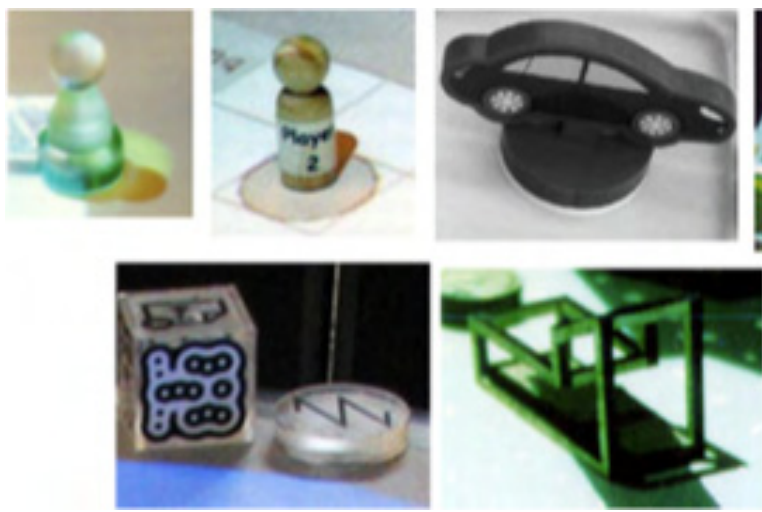
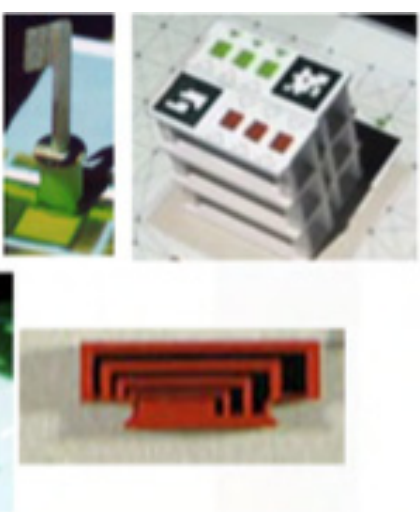

Fig. 9.1 Iconic tokens with different appearance. Top row: figurines representing people made of different materials (glass, wood), a car resembles a toy (LinguaBytes [94]), a bus stop, a storage tower (Tinkersheets [267]). Lower row: more abstract music manipulatives (ReacTable), an outline of a building (an early version of Urp [241]), and a token that represents a graphic transition between video clips (Tangible Video Editor [264]).

Fitzmaurice hypothized that specialized devices perform better than generic devices in space-multiplexed setups and experimentally proved that it speeds up inter-device acquisition. In their study of the GeoTUI system Couture et al. [42] show that a specialized device (a ruler) which offers additional physical constraints and affordances resulted in a better performance for a cutting-plane task in geophysics than a more general device, a two-puck prop.

Tangible objects can have specialized shapes, colors, weight, and material properties. They can also have space for annotations (see e.g., [51, 92]). By varying these parameters, designers and users can create meaningful expressions. A token might be light or heavy, creating different affordances for lifting and moving it. Slight changes in form often affect the ways users handle objects. For example, rounded edges increase the likelihood of a block to be playfully rotated upon a surface. The distribution of weight also has a strong effect on object handling, determining which side people are likely to hold up. Different object sizes further result in a different type of grip [151]. As a rule of thumb, square blocks with a width of $5-10 \mathrm{~cm}$ are easy to hold, a width of $5 \mathrm{~cm}$ supports a precision grip (pinching with thumb and one or two fingers) and a width of more than $10 \mathrm{~cm}$ requires a power grip with 
the entire hand. A token's weight can furthermore add meaning to the interaction - heaviness might indicate a more important and valuable object. A central affordance of physical objects is that they provide tactile feedback, supporting eyes-free control when performing fine-grained operations such as rotating the object, or moving two objects in relation to each other [135]. Moreover, an object's design can invite users to interact, pose a promise of pleasure - besides affordances objects may be "irresistables" [176].

A careful investigation of object affordances, e.g., through studying how people hold and handle different shapes, can guide the design of the physical form of tangible interfaces (cf. [62, 221]). Unfortunately, so far there is no systematic overview of which features of an object create what kinds of affordances, as there are too many parameters to be considered. In addition, according to Gibson [76], the affordances of an object exist relative to the action capabilities of an actor. For example, the rule of thumb on blocks with face sizes of $5 \mathrm{~cm}$ supporting a precision grip only holds for an average adult user, not for children, which have smaller hands.

\title{
9.2 Limitations
}

Several of the apparent limitations of TUIs result from the following challenge:

\begin{abstract}
"Such systems require a careful balance between physical and graphical expression to avoid physical clutter, and to take advantage of the contrasting strengths of different representational forms. This balance between physical and digital representations stands as one of TUI's greatest design challenges." [238].
\end{abstract}

Following, we discuss some of the current limitations of TUIs. While it is important to consider the limitations of TUIs when designing applications for specific domains, these limitations could also be viewed as challenges to be addressed by future research. 


\subsubsection{Scalability and the Risk of Loosing Physical Objects}

One of the biggest challenges for TUIs is scalability. Successful applications for small problems or data sets often do not scale up to complex problems involving many parameters and large data sets. With any application that attempts to provide a tangible representation this will be an issue, as larger representations take up more space. E.g. the tangible programming applications described in Section 4.4 support development of programs with a few lines of code. Envisioning the same programming mechanism for an industrial programming project is impossible.

The problem of scalability can be traced to several underlying reasons. The first would be the space physical tokens take up. In GUI design this is referenced as 'screen estate', and with TUIs the analog question would be how many tokens can be placed on an interactive surface. If the surface size would grow, it would become more difficult for a user to access and move tokens since they would need to walk through or fly over the representation. Related is the issue of physical clutter [65]. If the surface is already cluttered with the maximum number of tokens, there is no space for further tokens. Furthermore, physical objects need to be stored somewhere even if not in use, potentially requiring a large cabinet, and may get mislaid and lost (cf. [135]).

Scalability can also be interpreted literally. With a digital map one can zoom in to get a scaled up view of a subarea, and zoom out to see the map area in relation to its surroundings. With a physical model, the size of each element is fixed. Increasing the size of one element would require enlarging everything else and moving it apart, which would essentially require building a new model. Edge and Blackwell [52] describe this as bulkiness. A side-effect of physical bulkiness is that if one wants to work on two different problems at the same time, one needs to clean up to make space. Digital representations can simply be saved and re-opened, they do not take up space. Physical models thus can make it difficult to compare alternatives, lacking what is termed juxtaposability in the Cognitive Dimensions framework [52].

Having built up an architectural model in Urp, at some point one might decide to add a building to the left of the structure. Depending 
on how the interactive surface has been filled up, this might require shoveling the existing model to the right. With a GUI system, the user would be able to extend the canvas or to simply move the view. Scalability is thus related to mutability which we will discuss in more detail. It also contributes to what Edge and Blackwell [52] term premature commitment. To start building a model one needs to decide, e.g., on the size of objects and to select a location to place them, and later changes are hard.

Bellotti et al. [16] point out another potential issue related to scalability. TUIs are based on the notion of direct coupling, where tangible objects embody both the means of representation and the means of manipulating digital data [238]. TUIs thus provide an extreme form of direct manipulation (cf. [15]). Bellotti et al. [16] argue that with direct manipulation interfaces it takes more effort to issue complex commands. Direct manipulation does not have a concept of open variables and wildcards that command line interfaces provide, where they enable a global replace-all, and it does not adequately support manipulations of several objects.

\subsubsection{Versatility and Malleability}

Poupyrev et al. [189] point out that while digital objects are malleable, easy to create, modify, replicate, and distribute, physical objects are rigid and static. Iconic appearance and use of physical form to create affordances tend to result in concrete and specialized objects. As physical objects are not mutable, the system cannot transform these into different objects or alter their physical properties (e.g., change their color) even if such change might be appropriate. Specificness of objects, while promoting learnability, conflicts with abstraction and versatility. Another issue related to versatility and malleability is the difficulty of supporting an automatic undo, a history function, or a replay of actions (cf. [135]).

Considering versatility further, a graphical user interface can be used to perform a variety of tasks such as writing a document, chatting with friends, and playing music. However, a TUI is typically designed to facilitate a limited set of tasks. Furthermore, a graphical user interface 
allows users to switch between different views or representations (e.g., between a map and a satellite image). With a TUI such change is difficult, since the tangible representation has been chosen. While this limitation can be partially addressed through digital projection, there are cases in which a different view might retain the topology, but not the spatial geometry (e.g., compare the London tube map against a street map). Jacob et al. [119] claim that designers should aspire to create an interface that is as reality-based as possible (i.e., draw upon users' knowledge of skills of the real non-digital world). However, when the realism of an interface decreases its versatility, the designer should

make an explicit tradeoff between realism and versatility, only giving up on the realism of an interface in return for additional value to the user.

\subsubsection{User Fatigue}

The effects of size and weight of tangible objects should not be underestimated. Physical design should thus not just consider affordances in terms of what actions are invited through physical shape, but also the ergonomics and longer-term strain of the manual activity needed to perform actions.

For example, tangible tokens need to be easy to grasp, lift, and position: "TUI tokens are constantly lifted and moved about as their primary modality of interaction" [235]. A block with a width of $10 \mathrm{~cm}$ requires a power grip with the entire hand. Interaction using a TUI tends to require larger and different movements than traditional mousekeyboard interaction (which have their own problems, for example the rapid strain syndrome). Needing to stretch out over a surface repeatedly can become tiring over time, where smaller mouse movements take less effort. Moreover, physical size of the user in relation to the interaction surface determines reach. On the other hand, we should remember that physical interaction does not always need to be easy and effortless. On the contrary, people enjoy a challenge in games and the feeling of skillful mastery of a musical instrument (cf. [48]). 


\section{Research Directions}

As TUIs is an emerging and evolving field of research, many research issues require further study. Following, we highlight five such research directions both within core TUI research and in the wider research area of tangible interaction.

\subsection{Actuation}

In 2003, Koleva et al. [142] conducted an analysis of the types of coupling between the physical and digital and compared this with systems available at the time, revealing an asymmetry. Whereas many systems used physical objects to control digital objects, only few examples of "tangibles that push back" were found. Most systems supported only one-way communication, either being an ambient display or a data manipulation tool. Although actuation had been a part of the vision of TUIs from the very start, given the technical difficulties, it is only lately emerging as a strong trend. It is an important means for increasing the malleability of physical objects, which normally are rigid and static [189].

As a research area, actuated TUIs overlap with robotics and electromechanics, making use of similar principles and hardware. It also overlaps with research on organic user interfaces, when employing tran- 
sitive materials that can change their shape and perform some part of computation themselves [39]. Furthermore, artists experiment with selfactuation and designers explore the use of movement in product design (see e.g., [40, 47, 56, 203]).

Early examples of actuated TUIs include the peek-a-boo surrogates [83] which are rotated by a servo motor, and the Navigational Blocks [32] which are equipped with orientation sensors and electromagnets, and programmed to attract or repel each other to give actuated feedback on whether a particular configuration yields any output for a database search. The Actuated Workbench [177] used magnetic force to move objects on a table. A later incarnation of the same idea is Pico [181], a TUI that can sense and move small objects on top of a surface. This type of system is described by Poupyrev et al. [189] as self-rearranging displays that consist of multiple parts that rearrange themselves in space, in contrast to shape displays that directly create $3 \mathrm{D}$ physical shape.

Actuated tangibles with autonomous behavior can also be interpreted as robots. Jacobsson et al. [121] present the see-Puck and the GlowBots, a collection of LED displays on autonomously moving robotic platforms that communicate with each other, resulting in a continuously changing LED pattern on the surface and reacting to people picking up and relocating them. Robotic tangibles might extend to an architectural scale: Biloria [19] presents a series of experiments developing real-time interactive spatial prototypes, using pneumatics for actuation e.g., shape-shifting furniture and room units.

As an example of shape-shifting displays, Poupyrev et al. [189] present Lumen, a low resolution $13 \times 13$ pixel bit-map display where each pixel can individually move up and down. This enables a moving physical shape or texture augmenting a $2 \mathrm{D}$ visual display as well as tangible 3D controls that can literally be pressed down. SproutI/O [37] combines textiles and shape-memory alloys to create a kinetic display from soft textile that can sense touch.

The Linguabytes project [94] provides a nice example of using actuation in a subtle way, almost unnoticeably easing a task. This learning system helps multi-handicapped toddlers with motor impairment. It directs their hand when they place a physical piece on a platform (if 
the RFID tag is detected, electromagnets pull the piece into place) and has a slider that automatically limits range depending on its position. Other TUIs employ actuation, e.g., as vibrotactile feedback on information being detected and sucked into a handheld device [265].

Actuation, being the technical basis for product movement, is investigated also in product design, where movement (or "behavioral expression") provides a means to increase product expressiveness $[47,56]$. Movement can make abstract shapes come seemingly alive, as human perception has an intrinsic tendency for animism [245]. For example, the Mac laptop's softly and rhythmically blinking LED gives the impression of breathing. Products can also move physically, movement constituting a fourth dimension of design. As elements of a grammar of object movement Young et al. [260] identify path, volume, direction and velocity, and differentiate organic, random, and mechanic movement. Product movement can even express emotions through variation in speed, acceleration, type and volume of path, rhythm, and randomness [47].

\subsection{From Tangible User Interfaces to Organic User Interfaces}

Several recent approaches have attempted to move TUIs from employing a collection of rigid discrete objects to using organic and malleable materials. Driven by advances in flexible input technologies, electrophoretic ink, and shape changing materials, this emerging type of TUIs offers rapid form giving combined with computational feedback $[99,115]$. Rather than using predefined discrete objects with fixed forms, organic TUIs utilize continuous material that couples sensing and display. Examples of organic TUIs include Illuminating Clay [188] and SandScape [115]. Both are landscape sculpting and simulation TUIs. An emerging class of Kinetic Tangible Organic UIs record motion and gestures directly from the human body and replay them, creating a sense of a living organism [178]. For example, Topobo [196] is a $3 \mathrm{D}$ constructive assembly with kinetic memory. Though made of rigid material, some of its parts possess the ability to record and playback 
physical motion in 3D space. The user constructs an object using static and dynamic components and then 'teaches' the object how to move by physically manipulating the object itself.

Encompassing Organic TUIs, the vision of Organic User Interfaces (OUIs) refers to user interfaces with non-planar displays that may actively or passively change shape via continuous physical inputs $[38,246]$. The term 'organic' was selected because of the organic electronics technology and as a reference to the inspiration drawn from flexible, transformable, and evolvable organic shapes.

Examples for early organic user interfaces include multi-touch surfaces $[163,46,159]$, paper-based devices $[100,206]$, and actuated fabrics $[37,38]$. The vision of OUIs is strongly influenced by highly related areas of HCI research such as TUIs, Ubiquitous and Context-Aware Computing, Wearable Computing, Augmented Reality, and Multitouch Input. It overlaps with other future directions of TUI research such as actuation and full-body interaction, and incorporates concepts that emerged in the last two decades of HCI research such as RealityBased Interaction [119] and embedded interaction. With the understanding that in the future computers can take any form, be made from new computational materials, integrate sensing and display, and change their shape over time, many research directions and opportunities for innovation emerge. These include the development of new input technologies that are flexible and multi-touch, the development of actuating technologies that allow for devices and materials to actively re-shape themselves on multiple scales, and re-evaluation of interaction design and user experience in a world where computation is interleaved into the fabric of the real physical world. In the search for new computational materials, paper and fabric-based tangibles constitute a recent trend $[26,38,194]$.

\subsection{From Tangible Representation to Tangible Resources for Action}

Fernaeus, Tholander and Jonsson [58, 59] identify a shift in research trends from an information-centric to an action-centric perspective on 
tangible interaction. Due to some of the limitations of TUIs discussed earlier, in particular those of scalability and malleability, the design ideal of a tangible representation of digital data where "digital data is made tangible" [238] is hard to attain for larger systems. System designers thus now start to think about tangible objects as "resources for action" within a context of human sense-making $[58,59]$ and to focus on what the system enables users to do and the qualities of this activity [48]. This entails a focus on the social and contextual aspects of interaction, that is, seeing interaction as something that is not just between one user and the system but also occurs around the system $[59,105]$.

Tangible artifacts can act as resources for action in several ways [58]. They allow for a very wide range of actions and can be manipulated independent of the system ('offline' or for other purposes). This makes them resources for physical manipulation, for example in making use of epistemic action [137]. Tangibles support referential, social, and contextually oriented action, making it easy to make references by pointing, touching, or performing publicly visible actions. They can be a shareable resource. Tangibles can also be resources for perception and sensory experience, allowing for bodily engagement. Finally, tangibles can provide means for controlling and performing with a digital system, being resources for digitally mediated action. Importantly, an action-centric perspective then includes action directed toward the computer as well as offline socially oriented action, as well as sensory and experiential aspects [58].

With Patcher [57], tangible objects (programming cards) were primarily used to initiate actions, and had only a transient role as representation. Some of the cards act as tools for adding code, selecting a position within the simulation being programmed. Cards were often sorted in offline mode and handed around, negotiating what to do, and thereby supporting social interaction. With the BodyBug [164], the main aim of the system is to make the user move. There is no data in the normal sense and the system is essentially an enabler of activity. Playful TUIs such as the Tangicam [144] or IOBrush [205] often tend to be more enablers of activity than representations of information. 


\subsection{Whole-Body Interaction and Performative Tangible Interaction}

The perspective of tangible interaction entails whole-body interaction [105]. However, with a traditional TUI users tend to only manipulate objects within arms' reach using their hands and arms. Emerging systems allow users to interact with large objects within a large space, requiring full-body movement. Or, turn the user's body into an input device that is tracked in space. Whole-Body Interaction is related to what is referred to as "movement-based interaction" in product design [110]. Whole-Body Interaction has become a rapidly growing trend through new technologies such as the Wii and other technologies (e.g., in computer vision) that support gestural interaction.

Performativity is argued to be improved by TUIs in general $[105,124]$, because manipulation of tangible objects is more legible for observers than GUI interaction. Due to its increased public visibility, whole-body interaction tends to enhance performativity.

Movement-based interaction aims to enrich the interaction, in particular the aesthetics of movement, as well as to support bodily skills $[29,110]$. Instead of putting the entire burden of interaction on human cognition, with little diversity in required movements, some of the task might be delegated to perceptual-motor skills. For example, by mounting a rotary control which can also be slid sideways to the door of a microwave, the user can simultaneously set the power and cooking time while closing the door [47]. For an experienced user these actions will melt into one smooth single action and they might develop a motor memory for frequent settings. Movement can also be an aim in itself. The Bodybug [164] uses product actuation to invite whole-body interaction. It consists of a hand-sized motorized object that can climb along a rope that runs through its body in reaction to acceleration and angle of the rope. To play with the Bodybug, one attaches the rope (which has Velcro loops at the ends) to one's own body and begins to move. This initiates a dialog of movement between the user and the bug.

Large or furniture-sized tangible objects engender whole-body interaction. For example, Squeeze [186] is multi-person interactive furniture that allows people to interact with photos projected to the wall. 
Movement on the soft chair and pressure on its active zones influence the pictures. Kabisch et al. [127] discuss experiences with SignalPlay, an interactive sound environment where visitors to an exhibition are invited to move large tangible objects that influence a soundscape. Objects include bongos, a Star Wars lightsaber giant chess pieces, and oversized building blocks. Both Squeeze and SignalPlay require participants to collaborate for certain effects due to the size of objects.

Classical TUI examples for systems supporting performance include the reacTable [125], mediacrate [13], and mixiTUI [183]. Sheridan and Bryan-Kinns [222] derive design requirements for performative tangible interaction based on their design and performance experience with the iPoi. These are balls suspended from long strings, that when swung and spun around the body, create light and sound patterns. iPoi Systems have been used in interactive performances in clubs and during music festivals, where the audience was invited to participate. From these experiences it emerged that important properties for such systems are intuitiveness, unobtrusiveness, enticing passers-by to interact, being easily portable and installable, robust, and flexible, that is adjustable for different environments. In the context of performance, visibility is identified as key, allowing the audience to see what is going on and to learn simple routines from observation, enabling them to join in.

\subsection{Aesthetics}

Slowly but continuously we witness another TUI research theme evolving: the aesthetics of form and interaction. This research direction started with Ishii's emphasis of the physical qualities of tangible devices. In the previous sections we introduced research on the aesthetics of movement, either of the user or of a moving object, and presented work related to the form factors of TUIs [29, 47, 110, 158, 176, 260]. Furthermore, arts-based research [40, 203, 228] often aims for a poetic aesthetic that goes beyond form.

\subsection{Long-Term Interaction Studies}

Most evaluation studies of TUIs tend to be lab-based, having subjects interact with the system for a short time period, or take the form of 
a field trial. Such field trials also tend to have a short-time frame, sometimes involving researchers bringing the system in several times for a few hours over a couple of weeks, sometimes leaving a system in place for a few days. While such studies provide researchers with invaluable information about the ease of use for novice users and the initial attractiveness of the system, many other questions remain open, in particular:

- Is the system still interesting after the first excitement has worn off (novelty effect)? Is it engaging over a longer time period? (Or is it, like a fancy all-bells-and-whistles toy thrown into the corner after a while?).

- Is the system useful in supporting ongoing work? This question cannot be answered through a quick trial, as some issues in how well the system integrates with other systems or work organization may only become apparent over due course.

- Does the system support expert users and allow them to develop skill in using it? A short user trial or field test will give evidence of how easy it is to start using a system ('low floor', a low-entry threshold) but will not provide much insight into what level of complexity it supports (wide walls or high ceiling).

- How is the system adapted, adopted, and appropriated? A short field trial might provide initial insights, but only a longer deployment will tell whether and how users appropriate the system. This refers to changes in how they use the system, aspects of tailoring or end-user programming, and whether they might change their work (play/learning, etc.) processes.

Tackling these questions is important for demonstrating the practical value of TUIs. With the underlying technology becoming more mature, long-term interaction studies have now become feasible, the presence of the researcher not being required anymore. The first longterm studies are thus getting published (e.g., [51]), and field trials become more common and more extended. This also affects the 
methodology used. With an extended trial of a system, researchers will not want to videotape and analyze everything. Instead, researchers might decide to interview participants periodically, or ask participants to keep a diary or blog. 
In the last decade TUI research has become an established research area in HCI, as attested by the growing body of work and the number of venues dedicated for TUI research. Seeking to provide seamless interfaces between people, digital information, and the physical environments, TUI research shows a potential to enhance the way in which people interact with and leverage digital information. However, TUI research is still in its infancy. Extensive research is required to better understand the implications of interlinking the physical and digital worlds, to design tangible interaction techniques for complex real-world application domains, and to develop technologies that bridge the digital and physical worlds.

In this monograph, we presented a survey of this rich and diverse field of research. We gave an overview of the body of knowledge with respect to designing, implementing, and evaluating TUIs. We also highlighted perspectives from HCI, design, engineering, psychology, and cognitive sciences. In Section 10, we charted some possible directions for future work:

- actuation,

- from tangible user interfaces to organic user interfaces, 
- from tangible representation to tangible resources for action,

- whole-body interaction and performative tangible Interaction, and

- long-term interaction studies.

This review shows that work is already underway in these directions, but is still dispersed. We hope that this monograph helps to shed light on the work done in the field of TUIs in the last two decades, highlights many of the salient issues in this field, and will help practitioners and researchers alike to explore and contribute to this interdisciplinary and exciting field of research. 


\section{Acknowledgments}

We thank Paul Marshall, Michael Horn, and Rob Jacob for discussing the content of this monograph. Martin Kaltenbrunner assisted us on the characterization of musical tangibles and provided technical details about the ReacTIVision and TUIO. We also thank the many researchers that allowed us to use images of their work and provided us with such images and Megan Strait for helping to prepare this manuscript for print. 


\section{References}

[1] R. Abrams, "Adventures in tangible computing: The work of interaction designer 'Durrell Bishop' in context," Master's thesis, Royal College of Art, London, 1999.

[2] D. Africano, S. Berg, K. Lindbergh, P. Lundholm, F. Nilbrink, and A. Persson, "Designing tangible interfaces for children's collaboration," in Proceedings of CHIO4 Extended Abstracts, pp. 853-886, ACM, 2004.

[3] R. Aish, "3D input for CAAD systems," Computer Aided Design, vol. 11, no. 2, pp. 66-70, 1979.

[4] R. Aish and P. Noakes, "Architecture without numbers," Computer Aided Design, vol. 16, no. 6, pp. 321-328, 1984.

[5] M. W. Alibali, S. Kita, and A. Young, "Gesture and the process of speech production: We think, therefore we gesture," Language $\& 5$ Cognitive Processes, vol. 15, pp. 593-613, 2000.

[6] A. N. Antle, "The CTI framework: Informing the design of tangible systems for children," in Proceedings of TEI '07, pp. 195-202, NY: ACM, 2007.

[7] A. N. Antle, N. Motamedi, K.Tanenbaum, and Z. L. Xie, "The EventTable technique: Distributed fiducial markers," in Proceedings of TEI '09, pp. 307313, NY: ACM, 2009.

[8] D. Avrahami and S. Hudson, "Forming interactivity: A tool for rapid prototyping of physical interactive products," in Proceedings of DIS'02, pp. 141-146, NY: ACM, 2002.

[9] R. Balakrishnan and K. Hinckley, "The roles of kinesthetic reference frames in two-handed input performance," UIST'99 Symposium on User Interface Software and Technology, pp. 171-178, NY: ACM. 
[10] R. Ballagas, F. Memon, R. Reiners, and J. Borchers, "iStuff mobile: Rapidly prototyping new mobile phone interfaces for ubiquitous computing," in Proceedings of CHI '07, pp. 1107-1116, NY: ACM, 2007.

[11] R. Ballagas, M. Ringel, M. Stone, and J. Borchers, "iStuff: A physical user interface toolkit for ubiquitous computing environments," in Proceedings of CHI '03, pp. 537-544, NY: ACM, 2003.

[12] M. Banzi, Getting Started with Arduino. OReilly, 2009.

[13] T. Bartindale, J. Hook, and P. Olivier, "Media Crate: Tangible Live Media Production Interface," in Proceedings of TEI09, pp. 255-262, NY: ACM, 2009.

[14] M. Baskinger and M. Gross, "Tangible Interaction = Form + Computing," Interactions, vol. xvii.1, pp. 6-11, 2010.

[15] M. Beaudouin-Lafon, "Instrumental interaction: An interaction model for designing post-WIMP user interfaces," in Proceedings of CHI'00, pp. 446-453, NY: ACM, 2000.

[16] V. Bellotti, M. Back, W. Edwards, R. Grinter, A. Henderson, and C. Lopes, "Making sense of sensing systems: Five questions for designers and researchers," in Proceedings of CHI02, pp. 415-422, NY: ACM, 2002.

[17] S. Benford et al., "Expected, sensed and desired: A framework for designing sensing-based interaction," ACM Transactions on Computer-Human Interaction, vol. 12, no. 1, pp. 3-30, 2005.

[18] M. Billinghurst, H. Kato, and I. Poupyrev, "The MagicBook - Moving seamlessly between reality and virtuality," IEEE Computer Graphics and Applications, pp. 1-4, May/June 2001.

[19] N. Biloria, "Spatializing real time interactive environments," in Proceedings of TEI0\%, pp. 215-222, NY: ACM, 2007.

[20] A. Blackwell, D. Edge, L. M. Dubuc, J. A. Rode, M. Stringer, and E. F. Toye, "Using solid diagrams for tangible interface prototyping," IEEE Pervasive Computing, pp. 18-21, October-December 2005.

[21] A. Blackwell and R. Hague, "AutoHAN: An architecture for programming the home," in Proceedings of the IEEE Symposia on Human-Centric Computing Languages and Environments, pp. 150-157, 2001.

[22] S. Brave and A. Dahley, "inTouch: A Medium for Haptic Interpersonal Communication," in Extended Abstracts of CHI '97, pp. 363-364, NY: ACM, 1997.

[23] J. Brewer, A. Williams, and P. Dourish, "A handle on whats going on: Combining tangible interfaces and ambient displays for collaborative groups," in Proceedings of TEI0\%, pp. 3-10, NY: ACM.

[24] F. W. Bruns, "Zur Rückgewinnung von Sinnlichkeit. Eine neue Form des Umgangs mit Rechnern," Technische Rundschau, vol. 29, no. 39, pp. 14-18, 1993.

[25] W. Bruns and V. Brauer, "Bridging the gap between real and virtual modeling - A new approach to human-computer interaction," in Proceedings of the IFIP 5.10 Workshop on Virtual Prototyping, Providence, September, 1994, IFIP, 1996.

[26] L. Buechley, M. Eisenberg, J. Catchen, and A. Crockett, "The LilyPad Arduino: Using computational textiles to investigate engagement, aesthetics, and diversity in computer science education," in Proceedings of CHIO8, pp. 423-432, NY: ACM, 2008. 
[27] B. E. Bürdek, Design: History, Theory and Practice of Product Design. Birkhäuser Basel, 2005.

[28] A. Butz, M. Schmitz, A. Krüger, and H. Hullmann, "Tangible UIs for media control probes into the design space," in CHI 05 Extended Abstracts, pp. 957971, NY: ACM, 2005.

[29] J. Buur, M. V. Jensen, and T. Djajadiningrat, "Hands-only scenarios and video action walls: Novel methods for tangible user interaction design," in Proceedings of DIS04, pp. 185-192, NY: ACM, 2004.

[30] B. Buxton, Sketching User Experiences: Getting the Design Right and the Right Design. Morgan Kaufmann Publishers Inc, 2007.

[31] W. Buxton and B. Myers, "A study in two-handed input," in Proceedings of CHI'86: ACM Conference on Human Factors in Computing Systems, pp. 321$326,1986$.

[32] K. Camarata, E. Y. Do, B. R. Johnson, and M. D. Gross, "Navigational blocks: Navigating information space with tangible media," in Proceedings of the 7th International Conference on Intelligent User Interfaces, pp. 31-38, NY: IUI '02. ACM, 2002.

[33] K. Camarata, M. Gross, and E. Y. Do, "A physical computing studio: Exploring computational artifacts and environments," in International Journal of Architectural Computing, vol. 1, no. 2, pp. 169-190, 2004.

[34] A. Chang, B. Resner, B. Koerner, X. Wang, and H. Ishii, "LumiTouch: An emotional communication device," in Proceedings of CHI'01 Extended Abstracts, pp. 313-314, NY: ACM, 2001.

[35] H. Chung, C. J. Lee, and T. Selker, "Lover's cups: Drinking interfaces as new communication channels," in Proceedings of CHI'06, NY: ACM, 2006.

[36] A. Clark, Being There: Putting Brain, Body and World Together Again. Cambridge MA: MIT Press, 1997.

[37] M. Coelho and P. Maes, "Sprout I/O: A texturally rich interface," in Proceedings of TEI08, pp. 221-222, NY: ACM, 2008.

[38] M. Coelho and P. Maes, "Shutters: A permeable surface for environmental control and communication," in Proceedings of TEI '09, pp. 13-18, NY: ACM, 2009 .

[39] M. Coelho, I. Poupyrev, S. Sadi, R. Vertegaal, J. Berzowska, L. Buechley, P. Maes, and N. Oxman, "Programming reality: From transitive materials to organic user interfaces," in Proceedings of CHIO9 extended abstracts, pp. 47594762, NY: ACM, 2009.

[40] J. Coffin, "Robotany and Lichtung: A contribution to phenomenological dialogue," in Proceedings of TEI08, pp. 217-220, NY: ACM, 2008.

[41] J. Cohen, M. Withgott, and P. Piernot, "Logjam: A tangible multi-person interface for video logging," in Proceedings of CHI99, pp. 128-135, NY: ACM, 1999.

[42] N. Couture, G. Rivière, and P. Reuter, "GeoTUI: A tangible user interface for geoscience," in Proceedings of TEI08, pp. 89-96, NY: ACM, 2008.

[43] A. Cypher, ed., Watch What I Do: Programming by Demonstration. The MIT Press, 1993. 
[44] A. Damasio, The Feeling of What Happens: Body and Emotion in the Making of Consciousness. 1999.

[45] C. S. de Souza, The Semiotic Engineering of HumanComputer Interaction. Cambridge, MA: The MIT Press, 2004.

[46] P. Dietz and D. Leigh, "Diamondtouch: A multi-user touch technology," in UIST 01: Proceedings of the 14th Annual ACM Symposium on User Interface Software and Technology, pp. 219-226, NY: ACM, 2001.

[47] J. P. Djajadiningrat, B. Matthews, and M. Stienstra, "Easy doesn't do it: Skill and expression in tangible aesthetics," Personal and Ubiquitous Computing, vol. 11, no. 8, pp. 657-676, 2007.

[48] T. Djajadiningrat, K. Overbeeke, and S. Wensveen, "Augmenting fun and beauty: A pamphlet," in Proceedings of DARE2000, pp. S. 131-134, NY: ACM, 2000.

[49] T. Djajadiningrat, K. Overbeeke, and S. Wensveen, "But how, Donald, tell us how? On the creation of meaning in interaction design through feedforward and inherent feedback," in Proceedings of Designing Interactive Systems (DIS2002), pp. 285-291, NY: ACM, 2002.

[50] P. Dourish, Where the Action Is. The Foundations of Embodied Interaction. MIT Press, 2001.

[51] D. Edge and A. Blackwell, "Peripheral tangible interaction by analytic design," in Proceedings of TEI09, pp. 69-76, NY: ACM.

[52] D. Edge and A. Blackwell, "Correlates of the cognitive dimensions for tangible user interfaces," Journal of Visual Languages and Computing, vol. 17, no. 4, pp. 366-394, 2006.

[53] A. Ernevi, J. Redström, M. Redström, and L. Worbin, "The Interactive Pillows," in IT+Textiles, pp. 47-54, 2005.

[54] D. Fallman, "Wear, point and tilt: Designing support for mobile service and maintenance in industrial settings," in Proceedings of DIS2002, pp. 293-302, NY: ACM, 2002.

[55] M. Familant and M. Detweiler, "Iconic reference: Evolving perspectives and an organising framework," in International Journal of Man-Machine Studies vol. 39, pp. 705-728, 1993.

[56] L. Feijs, S. Kyffin, and B. Young, Proceedings of Design and Semantics of Form and Movement - DesForM 2005. Foreword. Koninklijke Philips Electronics N.V. Eindhoven. 3, 2005.

[57] Y. Fernaeus and J. Tholander, "Finding design qualities in a tangible programming space," in Proceedings of CHI06, pp. 447-456, NY: ACM, 2006.

[58] Y. Fernaeus, J. Tholander, and M. Jonsson, "Beyond representations: Towards an action-centric perspective on tangible interaction," International Journal of Arts and Technology, vol. 1, no. 3/4, pp. 249-267, 2008.

[59] Y. Fernaeus, J. Tholander, and M. Jonsson, "Towards a new set of ideals: Consequences of the practice turn in tangible interaction," in Proceedings of TEI'08, pp. 223-230, NY: ACM, 2008.

[60] J. Ferreira, P. Barr, and J. Noble, "The semiotics of user interface redesign," in Proceedings of the Sixth Australasian Conference on User interface - Volume 40, ACM International Conference Proceeding Series, vol. 104, pp. 47-53, Australian Computer Society, 2005. 
[61] K. Ferris and L. Bannon, "... A Load of ould Boxology!," in Proceedings of DIS 2002, pp. 41-49, N.Y.: ACM, 2002.

[62] A. Ferschau, S. Vogl, B. Emsenhuber, and B. Wally, "Physical shortcuts for media remote controls," in Proceedings of the Second International Conference on INtelligent TEchnologies for interaction enterTAINment, InteTain08, 2008.

[63] K. Fishkin, "A taxonomy for and analysis of tangible interfaces," Personal and Ubiquitous Computing, vol. 8, pp. 347-358, 2004.

[64] K. P. Fishkin, A. Gujar, B. L. Harrison, T. P. Moran, and R. Want, "Embodied user interfaces for really direct manipulation," Communications of the ACM, vol. 43, no. 9, pp. 75-80, 2000.

[65] G. W. Fitzmaurice, Graspable User Interfaces. Dissertation, Computer Science, University of Toronto, Canada, 1996.

[66] G. W. Fitzmaurice and W. Buxton, "An empirical evaluation of graspable user interfaces: Towards specialized, space-multiplexed input," in Proceedings of CHI97, pp. 43-50, NY: ACM, 1997.

[67] G. W. Fitzmaurice, H. Ishii, and W. Buxton, "Bricks: Laying the foundations for graspable user interfaces," in Proceedings of CHI95, pp. 442-449, NY: ACM, 1995.

[68] M. Fjeld, J. Fredriksson, M. Ejdestig, F. Duca, K. Bötschi, B. Voegtli, and P. Juchli, "Tangible user interface for chemistry education: Comparative evaluation and re-design," in Proceedings of CHI '0\%, pp. 805-808, NY: ACM, 2007.

[69] Fjeld, Bichsel, Rauterberg, "BUILD-IT: An intuitive design tool based on direct object manipulation," in Proceedings of International Gesture Workshop 1997\%, pp. 287-308, Berlin, Heidelberg, New York: Springer, 1997.

[70] J. Frazer, An Evolutionary Architecture. Themes VII. London: Architectural Association, 1995.

[71] J. Frazer and P. Frazer, "Intelligent physical three-dimensional modelling systems," in Proceedings of Computer Graphics'80, pp. 359-370, Online Publications, 1980.

[72] J. Frazer and P. Frazer, "Three-dimensional data input devices," in Proceedings of Computer Graphics in the Building Process, Washington: National Academy of Sciences, 1982.

[73] P. Frei, V. Su, B. Mikhak, and H. Ishii, "Curlybot: Designing a New Class of Computational Toys," in Proceedings of CHI 2000, pp. 129-136, NY: ACM, 2000.

[74] Furukawa, Fujihata, Muench, Small fish. http://hosting.zkm.de/wmuench/ small_fish, 2000.

[75] H. Gellersen, G. Kortuem, A. Schmidt, and M. Beigl, "Physcial prototyping with Smart-Its," IEEE Pervasive Computing, pp. 10-18, July-September 2004.

[76] J. J. Gibson, The Ecological Approach to Visual Perception. NY: Houghton Mifflin, 1979.

[77] S. Gill, "Developing information appliance design tools for designers," in Proceedings of the 1st Appliance Design Conference, Bristol, UK, 2003. 
[78] A. Gillet, M. Sanner, D. Stoffler, and A. Olson, "Tangible augmented interfaces for structural molecular biology," IEEE Computer Graphics \& Applications, vol. 25, no. 2, pp. 13-17, 2005.

[79] A. Girouard, E. T. Solovey, L. M. Hirshfield, S. Ecott, O. Shaer, and R. J. K. Jacob, "Smart blocks: A tangible mathematical manipulative," in Proceedings of TEI0\%, pp. 183-186, NY: ACM, 2007.

[80] S. Goldin-Meadow, Hearing Gesture: How Our Hands Help Us Think. Harvard University Press, 2003.

[81] S. Greenberg, "Collaborative physical user interfaces," in Communication and Collaboration Support Systems, (T. H. K. Okada and T. Inoue, eds.), pp. 24-42, Amsterdam, The Netherlands: IOS Press, 2005.

[82] S. Greenberg and C. Fitchett, "Phidgets: Easy development of physical interfaces through physical widgets," in Proceedings of UIST'01, pp. 209-218, NY: ACM, 2001.

[83] S. Greenberg and H. Kuzuoka, "Using digital but physical surrogates to mediate awareness, communication and privacy in media spaces," Personal Technologies, vol. 4, no. 1, Elsevier, January 2000.

[84] Y. Guiard, "Asymmetric division of labor in human skilled bimanual action: The kinematic chain as a model," The Journal of Motor Behavior, vol. 19, no. 4, pp. 486-517, 1987.

[85] D. Harel, "On visual formalisms," Communications of the ACM, vol. 31, no. 5, pp. 514-530, 1988.

[86] B. L. Harrison, K. P. Fishkin, A. Gujar, D. Portnov, and R. Want, "Bridging physical and virtual worlds with tagged documents, objects and locations," in Proceedings of CHI '99 Extended Abstracts, pp. 29-30, NY: ACM, 1999.

[87] B. Hartmann, L. Abdulla, M. Mittal, and S. R. Klemmer, "Authoring sensorbased interactions by demonstration with direct manipulation and pattern recognition," in Proceedings of CHI 'O7, pp. 145-154, NY: ACM, 2007.

[88] B. Hartmann, S. R. Klemmer, M. Bernstein, and N. Mehta, "d.tools: Visually prototyping physical UIs through statecharts," in Conference Supplement to UIST'2005.

[89] A. Hauptmann, "Speech and gestures for graphic image manipulation," in Proceedings of CHI89, pp. 241-245, NY: ACM, 1989.

[90] C. Heath and P. Luff, "Convergent activities - Line control and passenger information on the London underground," in Cognition and Communication at Work, (Y. Engeström and D. Middleton, eds.), pp. 96-129, Cambridge University Press, 1998.

[91] M. Heidegger, Being and Time. NY: Harper and Row, 1927. English translation 1962.

[92] M. Heijboer and E. van den Hoven, "Keeping up appearances: Interpretation of tangible artifact design," in Proceedings of NordiCHI 2008, pp. 162-171, NY: ACM, 2008.

[93] B. Hengeveld, C. Hummels, and K. Overbeeke, "Tangibles for toddlers learning language," in Proceedings of TEI09, pp. 161-168, NY: ACM, 2009.

[94] B. Hengeveld, C. Hummels, K. Overbeeke, R. Voort, H. van Balkom, and J. de Moor, "Let me actuate you," in Proceedings of TEI08, pp. 159-166, NY: ACM, 2008. 
[95] K. Hinckley, R. Pausch, J. Goble, and N. Kassel, "Passive real-world interface props for neurosurgical visualization," in Proceedings of CHI94, pp. 452-458, NY: ACM, 1994.

[96] K. Hinckley, R. Pausch, D. Proffitt, J. Patten, and N. Kassell, "Cooperative bimanual action," in Proceedings of the SIGCHI conference on Human factors in computing systems, pp. 27-34, NY: ACM, 1997.

[97] S. Hinske, M. Langheinrich, and M. Lampe, "Towards guidelines for designing augmented toy environments," in Proceedings of DIS 2008, pp. 78-87, NY: ACM.

[98] J. D. Hollan, E. Hutchins, and D. Kirsh, "Distributed cognition: A new foundation for human-computer interaction research," ACM Transactions on Computer-Human Interaction (TOCHI), vol. 7, no. 2, pp. 174-196, 2000.

[99] D. Holman and R. Vertegaal, "Organic user interfaces: Designing computers in any way, shape, or form," Communications of the ACM, vol. 51, no. 6, pp. 48-55, 2008.

[100] D. Holman, R. Vertegaal, and N. Troje, "PaperWindows: Interaction techniques for digital paper," in Proceedings of ACM CHI'05, pp. 591-599, NY: ACM, 2005.

[101] L. E. Holmquist, J. Redström, and P. Ljungstrand, "Token-based acces to digital information," in Proceedings of the 1st International Symposium on Handheld and Ubiquitous Computing, (H. Gellersen, ed.), pp. 234-245, Lecture Notes In Computer Science, vol. 1707, London: Springer-Verlag, 1999.

[102] M. S. Horn, E. T. Solovey, R. J. Crouser, and R. J. K. Jacob, "Comparing the use of tangible and graphical programming interfaces for informal science education," in Proceedings of CHI'09, pp. 975-984, NY: ACM, 2009.

[103] M. S. Horn, E. T. Solovey, and R. J. K. Jacob, "Tangible programming for informal science learning: Making TUIs work for Museums," in Proceedings of 'th International Conference on Interaction Design and Children IDC'08, pp. 194-201, NY: ACM, 2008.

[104] E. Hornecker, "Creative idea exploration within the structure of a guiding framework: The card brainstorming game," in Proceedings of TEI'10, pp. 101108, NY: ACM, 2010.

[105] E. Hornecker and J. Buur, "Getting a grip on tangible interaction: A framework on physical space and social interaction," in Proceedings of CHIO6, pp. 437-446, NY: ACM, 2006.

[106] E. Hornecker, R. Jacob, C. Hummels, B. Ullmer, A. Schmidt, E. van den Hoven, and A. Mazalek, "TEI goes on: Tangible and embedded interaction," IEEE Pervasive Computing Magazine/Journal, vol. 7, no. 2, pp. 91-95, AprilJune 2008.

[107] E. Hornecker and T. Psik, "Using ARToolKit markers to build tangible prototypes and simulate other technologies," in Proceedings of Interact 2005, pp. 30-42, Springer.

[108] C. J. Huang, E. Yi-Luen Do, and M. D. Gross, "MouseHaus table," in Proceedings of CAAD Futures, 2003.

[109] Y. Huang, M. D. Gross, E. Y. Do, and M. Eisenberg, "Easigami: A reconfigurable folded-sheet TUI," in Proceedings of TEI '09, pp. 107-112, NY: ACM, 2009. 
[110] C. Hummels, K. C. Overbeeke, and S. Klooster, "Move to get moved: A search for methods, tools and knowledge to design for expressive and rich movementbased interaction," Personal Ubiquitous Computing, vol. 11, no. 8, pp. 677690, 2007.

[111] J. Hurtienne and J. H. Israel, "Image schemas and their metaphorical extensions: Intuitive patterns for tangible interaction," in Proceedings of TEIO\%, pp. 127-134, NY: ACM, 2007.

[112] J. Hurtienne, J. H. Israel, and K. Weber, "Cooking up real world buisiness applications combining physicality, digitality, and image schemas," in Proceedings of TEI'08, pp. 239-246, NY: ACM, 2008.

[113] E. Hutchins, Cognition in the Wild. Cambridge, London: MIT Press. 3d Pressing, 1999

[114] E. Hutchins and L. Palen, "Constructing meaning from space, gesture, and speech," in Discourse, Tools, and Reasoning - Essays on Situated Cognition, Series F: Computer and System Sciences, Vol. 160, (L. B. Resnick, R. Säljö, C. Pontecorvo, and B. Burge, eds.), pp. S. 23-40, NATO ASI Series, 1993.

[115] H. Ishii, "The tangible user interface and its evolution," Communications of the ACM, vol. 51, no. 6, pp. 32-36, 2008.

[116] H. Ishii, C. Ratti, B. Piper, Y. Wang, A. Biderman, and E. Ben-Joseph, "Bringing clay and sand into digital design - Continuous tangible user interfaces," BT Technology Journal, vol. 22, no. 4, pp. 287-299, October 2004.

[117] H. Ishii and B. Ullmer, "Tangible bits: Towards seamless interfaces between people, bits and atoms," in Proceedings of CHI97, pp. 234-241, NY: ACM, 1997.

[118] S. Izadi, A. Butler, S. Hodges, D. West, M. Hall, B. Buxton, and M. Molloy, "Experiences with building a thin form-factor touch and tangible tabletop," in Proceedings of IEEE Tabletop '08, pp. 193-196, 2008.

[119] R. J. K. Jacob, A. Girouard, L. M. Hirshfield, M. S. Horn, O. Shaer, E. T. Solovey, and J. Zigelbaum, "Reality-based interaction: A framework for postWIMP interfaces," in Proceedings of CHI 2008, pp. 201-210, NY: ACM, 2008.

[120] R. J. K. Jacob, H. Ishii, G. Pangaro, and J. Patten, "A tangible interface for organizing information using a grid," in Proceedings of CHI '02, pp. 339-346, NY: ACM, 2002.

[121] M. Jacobsson, J. Bodin, and L. E. Holmquist, "The see-Puck: A platform for exploring human-robot relationships," in Proceedings of CHIO8, pp. 141-144, NY: ACM, 2008.

[122] M. V. Jensen and M. Stienstra, "Making sense: Interactive sculptures as tangible design material," in Proceedings of DPPI0\%, Designing Pleasurable Products and Interfaces, pp. 255-269, NY: ACM, 2007.

[123] Johnson, The body in the mind. The bodily basis of meaning, imagination, and reason, University of Chicago Press, 1987.

[124] S. Jordà, "On stage: The reactable and other musical tangibles go real," International Journal of Arts and Technology (IJART), vol. 1, no. 3/4, pp. 268-287, Special Issue on Tangible and Embedded Interaction 2008. 
[125] S. Jordà, G. Geiger, M. Alonso, and M. Kaltenbrunner, "The reacTable: Exploring the synergy between live music performance and tabletop tangible interfaces," in Proceedings of TEI '0\%, pp. 139-146, NY: ACM, 2007.

[126] B. Jordan and A. Henderson, "Interaction analysis foundations and practice," Journal of the Learning Sciences, vol. 4, no. 1, pp. 39-103, 1995.

[127] E. Kabisch, A. Williams, and P. Dourish, "Symbolic objects in a networked gestural sound interface," in Proceedings of CHIO5 extended abstracts, pp. 1513-1516, NY: ACM, 2005.

[128] J. J. Kalanithi and V. M. Bove, "Connectibles: Tangible social networks," in Proceedings of TEI08, pp. 199-206, NY: ACM, 2008.

[129] M. Kaltenbrunner, Website on Tangible Music. Read April 2009, http:// modin.yuri.at/tangibles/.

[130] M. Kaltenbrunner and R. Bencina, "reacTIVision: A computer-vision framework for table-based tangible interaction," in Proceedings of TEI 'O', pp. 6974, NY: ACM, 2007.

[131] H. Kato and M. Billinghurst, "Marker tracking and HMD calibration for a video-based augmented reality conferencing system," in Proceedings of the 2nd International Workshop on Augmented Reality (IWAR 99), 1999.

[132] H. Kato, M. Billinghurst, I. Poupyrev, N. Tetsutani, and K. Tachibana, "Tangible augmented reality for human computer interaction," in Proceedings of Nicograph 2001, Nagoya, Japan, 2001.

[133] H. Kato and M. Billinghurst et al., "Virtual object manipulation on a table-top AR environment," in Proceedings of International Symposium on Augmented Reality ISAR 2000, pp. 111-119, 2000.

[134] M. J. Kim and M. L. Maher, "The impact of tangible user interfaces on designers' spatial cognition," Human-Computer Interaction, vol. 23, no. 2, 2008.

[135] D. S. Kirk, A. Sellen, S. Taylor, N. Villar, and S. Izadi, "Putting the physical into the digital: Issues in designing hybrid interactive surfaces," in Proceedings of HCI 2009, 2009.

[136] D. Kirsh, "The intelligent use of space," Artificial Intelligence, vol. 73, no. 1-2, pp. 31-68, British Computer Society, 1995.

[137] D. Kirsh and P. Maglio, "On distinguishing epistemic from pragmatic actions," Cognitive Science, vol. 18, no. 4, pp. 513-549, 1994.

[138] S. R. Klemmer, B. Hartmann, and L. Takayama, "How bodies matter: Five themes for interaction design," in Proceedings of DIS2006 Conference on Designing Interactive Systems, pp. 140-149, NY: ACM, 2006.

[139] S. R. Klemmer, J. Li, J. Lin, and J. A. Landay, "Papier Mâché: Toolkit support for tangible input," in Proceedings of CHI2004, pp. 399-406, NY: ACM, 2004.

[140] S. R. Klemmer, M. W. Newman, R. Farrell, M. Bilezikjian, and J. A. Landay, "The designers outpost: A tangible interface for collaborative web site design," in Proceedings of UIST'2001: ACM Symposium on User Interface Software and Technology, pp. 1-10, NY: ACM, 2001.

[141] K. Kobayashi, M. Hirano, A. Narita, and H. Ishii, "A tangible interface for IP network simulation," in Proceedings of CHI '03 extended abstracts, pp. 800801, NY: ACM, 2003. 
[142] B. Koleva, S. Benford, K. H. Ng, and T. Rodden, A Framework for Tangible User Interfaces, Physical Interaction, (PI03) - Workshop on Real World User Interfaces, Mobile HCI Conference 2003, Udine, Italy, 2003.

[143] G. Kurtenbach, G. Fitzmaurice, T. Baudel, and B. Buxton, "The design of a GUI paradigm based on tablets, two-hands, and transparency," in Proceedings of CHI97, pp. 35-42, NY: ACM, 1997.

[144] J.-B. Labrune and W. Mackay, "Tangicam: Exploring observation tools for children," in Proceedings of IDC 2005, pp. 95-102, NY: ACM, 2005.

[145] G. Lakoff and M. Johnson, Metaphors We Live By. University of Chicago Press, 1980.

[146] B. Laurel, Computers as Theater. Addison-Wesley Professional, 1993.

[147] V. LeClerc, A. Parkes, and H. Ishii, "Senspectra: A computationally augmented physical modeling toolkit for sensing and visualization of structural strain," in Proceedings of CHI '07, pp. 801-804, NY: ACM, 2007.

[148] G. A. Lee, C. Nelles, M. Billinghurst, and G. J. Kim, "Immersive authoring of tangible augmented reality applications," in Proceedings of IEEE/ACM International Symposium on Mixed and Augmented Reality, pp. 172-181, IEEE Computer Society, 2004.

[149] J. Leitner, M. Haller, K. Yun, W. Woo, M. Sugimoto, and M. Inami, "IncreTable, a mixed reality tabletop game experience," in Proceedings of the 2008 International Conference on Advances in Computer Entertainment Technology, pp. 9-16, NY: ACM, 2008.

[150] W. E. Mackay and A.-L. Fayard, "Designing interactive paper: Lessons from three augmented reality projects," in Proceedings of IWAR98, International Workshop on Augmented Reality, Natick, MA, 1999.

[151] C. L. MacKenzie and T. Iberall, The Grasping Hand. North Holland, 1994.

[152] C. Magerkurth, M. Memisoglu, T. Engelke, and N. A. Streitz, "Towards the next generation of tabletop gaming experiences," in Graphics Interface 2004 (GI'04), pp. 73-80, AK Peters, 2004.

[153] A. Manches, C. O'Malley, and S. Benford, "Physical manipulation: Evaluating the potential for tangible designs," in Proceedings of TEI 09, NY: ACM, 2009.

[154] V. Maquil, T. Psik, and I. Wagner, "The ColorTable: A design story," in Proceedings of TEI '08, pp. 97-104, NY: ACM, 2008.

[155] N. Marquardt and S. Greenberg, "Distributed physical interfaces with shared Phidgets," in Proceedings of TEI 'O\%, pp. 13-20, NY: ACM, 2007.

[156] P. Marshall, "Do tangible interfaces enhance learning?" in Proceedings of TEI'07, pp. 163-170, NY: ACM, 2007.

[157] P. Marshall, S. Price, and Y. Rogers, "Conceptualizing tangibles to support learning," in Proceedings of IDC 2003, pp. 101-109, NY: ACM, 2003.

[158] E. S. Martinussen and T. Arnall, "Designing with RFID," in Proceedings of TEI 2009, pp. 343-350, NY: ACM, 2009.

[159] N. Matsushita and J. Rekimoto, "HoloWall: Designing a finger, hand, body, and object sensitive wall," in Proceedings of UIST9\%, pp. 209-210, NY: ACM, 1997.

[160] A. Mazalek and E. van den Hoven, "Framing tangible interaction frameworks. Tangible interaction for design special issue," AIEDAM journal, Spring 2009, vol. 23, pp. 225-235, 2009. 
[161] T. S. McNerney, "From turtles to tangible programming bricks: Explorations in physical language design," Pers Ubiquit Comput, vol. 8, pp. 326-337, 2004.

[162] M. Merleau-Ponty, Phenomnologie de la perception. Paris: Gallimard, 1945.

[163] Microsoft Corp. Microsoft Surface, http://www.microsoft.com/surface/, 2009.

[164] J. Moen, "From hand-held to body-worn: Embodied experiences of the design and use of a wearable movement-based interaction concept," in Proceedings of TEI'07, pp. 251-258, NY: ACM, 2007.

[165] E. Mugellini, E. Rubegni, S. Gerardi, and O. A. Khaled, "Using personal objects as tangible interfaces for memory recollection and sharing," in Proceedings of TEI'07, pp. 231-238, NY: ACM, 2007.

[166] B. A. Myers, "Why are human-computer interfaces difficult to design and implement?," Technical Report. UMI Order Number: CS-93-183., Carnegie Mellon University, 1993.

[167] H. Newton-Dunn, H. Nakano, and J. Gibson, "Blockjam: A tangible interface for interactive music," in Proceedings of the 2003 Conference on New Interfaces for Musical Expression (NIME-03), pp. 170-177, 2003.

[168] D. Norman, The Psychology of Everyday Things. New York: Basic Books, 1988.

[169] D. Norman, Things that Make Us Smart. Defending Human Attributes in the Age of the Machine. Reading, Mass, Addison Wesley, 1994.

[170] D. Norman, "Affordance, Conventions, and Design," Interactions, vol. 6, no. 3, pp. 38-43, 1999.

[171] D. Norman, "The next UI breakthrough, Part 2: Physicality," Interactions, vol. 14 , no. 4, pp. 46-47, 2007.

[172] D. R. Olsen, User Interface Management Systems: Models and Algorithms. Morgan Kaufmann, San Francisco, 1992.

[173] C. O'Malley and D. Stanton, "Tangible technologies for collaborative storytelling," in Proceedings of the 1st European Conference on Mobile and Contextual Learning, pp. 3-7, 2002.

[174] C. O'Malley and D. Stanton Fraser, "Literature review in learning with tangible technologies," NESTA futurelab report 12, Bristol, 2004.

[175] D. O'Sullivan and T. Igoe, Physical Computing: Sensing and Controlling the Physical World with Computers. Boston: Muska and Lipman, 2004.

[176] K. Overbeeke and S. Wensveen, "From perception to experience, from affordances to irresistibles," in Proceedings of DPPI03 (Designing Pleasurable Products and Interfaces), pp. 92-97, NY: ACM, 2003.

[177] G. Pangaro, D. Maynes-Aminzade, and H. Ishii, "The actuated workbench: Computer-controlled actuation in tabletop tangible interfaces," in Proceedings of UIST 2002 Symposium on User Interface Software and Technology, pp. 181190, NY: ACM, 2002.

[178] A. Parkes and H. Ishii, "Kinetic sketchup: Motion prototyping in the tangible design process," in Proceedings of TEI '09, pp. 367-372, NY: ACM, 2009.

[179] V. Parmar, G. Groeneveld, A. Jalote-Parmar, and D. Keyson, "Tangible user interface for increasing social interaction among rural women," in Proceedings of TEI 2009, pp. 139-145, NY: ACM, 2009. 
[180] J. Patten and H. Ishii, "A comparison of spatial organization strategies in graphical and tangible user interfaces," in Proceedings of Designing Augmented Reality Environments, DARE '00, pp. 41-50, NY: ACM, 2000.

[181] J. Patten and H. Ishii, "Mechanical constraints as computational constraints in tabletop tangible interfaces," in Proceedings of CHI'07, pp. 809-818, NY: ACM, 2007.

[182] J. Patten, B. Recht, and H. Ishii, "Audiopad: A tag-based interface for musical performance," in Proceedings of the International Conference on New Interface for Musical Expression NIME02, pp. 24-26, 2002.

[183] E. W. Pedersen and K. Hornbæk, "mixiTUI: A tangible sequencer for electronic live performances," in Proceedings of TEI '09, pp. 223-230, NY: ACM, 2009.

[184] C. S. Peirce, "Collected Papers of Charles Sanders Peirce," in 8 Volumes, (C. Hartshorne, P. Weiss, and A. Burks, eds.), Cambridge MA: Harvard University Press, 1931-1958.

[185] R. Perlman, Using Computer Technology to Provide a Creative Learning Environment for Preschool Children. MIT Logo Memo 24, 1976.

[186] M. G. Petersen, "Squeeze: Designing for playful experiences among co-located people in homes," in Proceedings of CHI 2007 Extended Abstracts, pp. 26092614, NY: ACM, 2007.

[187] C. Petri, "Kommunikation mit Automaten," Ph.D. thesis, University of Bonn, 1962.

[188] B. Piper, C. Ratti, and H. Ishii, "Illuminating clay: A 3-D tangible interface for landscape analysis," in Proceedings of CHI '02, pp. 355-362, NY: ACM, 2002.

[189] I. Poupyrev, T. Nashida, and M. Okabe, "Actuation and tangible user interfaces: The Vaucanson duck, robots, and shape displays," in Proceedings of Tangible and Embedded interaction, TEI 'O\%, pp. 205-212, NY: ACM, 2007.

[190] R. Poynor, "The hand that rocks the cradle," ID Magazine, pp. 60-65, May/June 1995.

[191] S. Price, "A representation approach to conceptualizing tangible learning environments," in Proceedings of TEI 2008, pp. 151-158, NY: ACM, 2008.

[192] S. Price and Y. Rogers, "Let's get physical: The learning benefits of interacting in digitally-augmented physical spaces," Journal of Computers and Education, vol. 15, no. 2, pp. 169-185, 2004.

[193] S. Price, Y. Rogers, M. Scaife, D. Stanton, and H. Neale, "Using tangibles to support new ways of playing and learning," in Proceedings of Interaction Design for Children 2002, 2002.

[194] J. Qi and L. Buechley, "Electronic popables: Exploring paper-based computing through an interactive pop-up book," in Proceedings of TEI '10, pp. 121-128, NY: ACM, 2010.

[195] H. Raffle, C. Vaucelle, R. Wang, and H. Ishii, "Jabberstamp: Embedding sound and voice in traditional drawings," in Proceedings of IDC, pp. 137-144, NY: ACM, 2007.

[196] H. S. Raffle, A. J. Parkes, and H. Ishii, "Topobo: A constructive assembly system with kinetic memory," in Proceedings of the ACM CHI'04, pp. 647654, NY: ACM, 2004. 
[197] J. Rekimoto and Y. Ayatsuka, "CyberCode: Designing augmented reality environments with visual tags," in Proceedings of DARE 2000, NY: ACM, 2000.

[198] M. Resnick, "Behavior construction kits," Communications of the ACM, vol. 36, no. 7, pp. 64-71, July 1993.

[199] M. Resnick, F. Martin, R. Berg, R. Borovoy, V. Colella, K. Kramer, and B. Silverman, "Digital manipulatives: New toys to think with," in Proceedings of CHI'98, pp. 281-287, NY: ACM, 1998.

[200] Y. Rogers and H. Muller, "A framework for designing sensor-based interactions to promote exploration and reflection in play," International Journal of Human Computer Studies, vol. 64, no. 1, pp. 1-14, 2006.

[201] Y. Rogers, M. Scaife, S. Gabrielli, I.-I. Smith, and E. A. Harris, "Conceptual framework for mixed reality environments: Designing novel learning activities for young children," Presence: Teleoperators and Virtual Environments, vol. 11, no. 6, pp. 677-686, 2002.

[202] T. Rohrer, "The body in space: Embodiment, experientialism and linguistic conceptualization," in Body, Language and Mind, vol. 2, (J. Zlatev, T. Ziemke, R. Frank, and R. Dirven, eds.), Berlin: Mouton de Gruyter, 2006.

[203] A. Rydarowski, O. Samanci, and A. Mazalek, "Murmur: Kinetic relief sculpture, multi-sensory display, listening machine," in Proceedings of TEI'08, pp. 231-239, NY: ACM, 2008.

[204] K. Ryokai and J. Cassell, "StoryMat: A play space for collaborative storytelling," in Proceedings of CHI'99, pp. 272-273, NY: ACM, 1999.

[205] K. Ryokai, S. Marti, and H. Ishii, "I/O brush: Drawing with everyday objects as ink," in Proceedings of CHI 2004, pp. 303-310, NY: ACM, 2004.

[206] G. Saul and M. D. Gross, "Co-designed paper devices," in Programming Reality: From Transitive Materials to Organic User Interfaces, a CHI 2009 Workshop, 2009.

[207] M. Scaife and Y. Rogers, "External cognition: How do graphical representations work?," International Journal of Human-Computer Studies, vol. 45, no. 2, pp. 185-213, 1996.

[208] K. Schäfer, V. Brauer, and W. Bruns, "A new approach to human-computer interaction - Synchronous modeling in real and virtual spaces," in Proceedings of DIS 1997, pp. 335-344, NY: ACM, 1997.

[209] B. Schiettecatte and J. Vanderdonckt, "AudioCubes: A distributed cube tangible interface based on interaction range for sound design," in Proceedings of TEI '08, pp. 3-10, NY: ACM, 2008.

[210] A. Schmidt and K. Van Laerhoven, "How to build smart appliances," IEEE Personal Communications, Special Issue on Pervasive Computing, vol. 8, no. 4, IEEE Press, pp. 66-71, 2001.

[211] D. Schön, Educating the Refective Practicioner. San Francisco, London: Jossey-Bass Publications, 1989.

[212] E. Schweikardt, N. Elumeze, M. Eisenberg, and M. D. Gross, "A tangible construction kit for exploring graph theory," in Proceedings of TEI '09, pp. 373376, NY: ACM, 2009.

[213] J. A. Seitz, "The bodily basis of thought," in Proceedings of 29th Annual Symposium of the Jean Piaget Society, Mexico City, Mexico, 1999. 
[214] O. Shaer, M. S. Horn, and M. S. Jacob, Tangible user interface laboratory: Teaching tangible interaction design in practice, AIEDAM Special Issue on Tangible Interaction for Design, 2009.

[215] O. Shaer and R. J. K. Jacob, "A specification paradigm for the design and implementation of tangible user interfaces," ACM Transactions on ComputerHuman Interaction, vol. 16, no. 4, pp. 251-261, 2009.

[216] O. Shaer, N. Leland, E. H. Calvillo-Gamez, and R. J. K. Jacob, "The TAC paradigm: Specifying tangible user interfaces," Personal and Ubiquitous Computing, vol. 8, pp. 359-369.

[217] O. Shaer, B. Ziraknejad, K. Camarata, E. Yi-Luen Do, and M. Gross A Computationally Enhanced Play Board for Group Interaction, a Hot Spot paper, Pervasive, 2004.

[218] E. Sharlin, Y. Itoh, B. Watson, Y. Kitamura, S. Sutphen, and L. Liu, "Cognitive cubes: A tangible user interface for cognitive assessment," in Proceedings of CHIO2, pp. 347-354, NY: ACM, 2002.

[219] E. Sharlin, B. Watson, Y. Kitamura, F. Kishino, and Y. Itoh, "On tangible user interfaces, humans and spatiality," Personal and Ubiquitous Computering, vol. 8, no. 5, pp. 338-346, 2004.

[220] H. Sharp, Y. Rogers, and J. Preece, Interaction Design. John Wiley, 2007.

[221] J. Sheridan and G. Kortuem, "Affordance-based design of physical interfaces for ubiquitous environments," in Proceedings of UCS06, pp. 183-199, Springer, 2006.

[222] J. G. Sheridan and N. Bryan-Kinns, "Designing for performative tangible interaction," International Journal of Arts and Technology (IJART), vol. 1, no. 3/4, pp. 288-308, 2008.

[223] L. Sherman, A. Druin, J. Montemayor, A. Farber, M. Platner, S. Simms, J. Porteous, H. Alborzi, J. Best, J. Hammer, A. Kruskal, J. Matthews, E. Rhodes, C. Cosans, and L. Lal, "StoryKit: Tools for children to build room-sized interactive experiences," in Extended Abstracts, Interactive Video Poster, CHI2001, NY: ACM, 2001.

[224] A. Singer, D. Hindus, L. Stifelman, and S. White, "Tangible progress: Less is more in somewire audio spaces," in Proceedings of CHI '99, pp. 104-111, NY: ACM, 1999.

[225] L. Sitorus, S. S. Cao, and J. Buur, "Tangible user interfaces for configuration practices," in Proceedings of TEI0\%, pp. 223-230, NY: ACM, 2007.

[226] W. Spiessl, N. Villar, H. Gellersen, and A. Schmidt, "VoodooFlash: Authoring across digital and physical form," in Proceedings of TEI 'O\%, NY: ACM, 2007.

[227] M. Stringer, E. F. Toye, J. Rode, and A. Blackwell, "Teaching rhetorical skills with a tangible user interface," in Proceedings of IDC 2004, pp. 11-18, NY: ACM, 2004.

[228] R. Strong and B. Gaver, "Feather, scent and shaker: Supporting simple intimacy," in Proceedings of CSCW 1996, pp. 29-30, NY: ACM, 1996.

[229] J. Sturm, T. Bekker, B. Groenendaal, R. Wesselink, and B. Eggen, "Key issues for the successful design of an intelligent, interactive playground," in Proceedings of IDC 2008, pp. 258-265, NY: ACM, 2008. 
[230] H. Suzuki and H. Kato, "AlgoBlock: A tangible programming language, a tool for collaborative learning," in Proceedings of the 4th European Logo conference (Eurologo93), pp. 297-303, Athens, Greece, 1993.

[231] H. Suzuki and H. Kato, "Interaction-level support for collaborative learning: AlgoBlock - An open programming language," in Proceedings of CSCL, 1995.

[232] L. Terrenghi, D. Kirk, H. Richter, S. Krämer, O. Hilliges, and A. Butz, "Physical handles at the interactive surface: Exploring tangibility and its benefits," in Proceedings of AVI 2008, pp. 138-145, NY: ACM, 2008.

[233] K. N. Truong, G. R. Hayes, and G. D. Abowd, "Storyboarding: An empirical determination of best practices and effective guidelines," in Proceedings of Designing interactive Systems DIS '06, pp. 12-2, NY: ACM, 2006.

[234] S. Turkle, Evocative Objects: Things We Think With. Cambridge, MA: MIT Press, 2007.

[235] B. Ullmer, "Tangible interfaces for manipulating aggregates of digital information," PhD Dissertation, Massachusetts Institute of Technology, 2002.

[236] B. Ullmer and H. Ishii, "Mediablocks: Tangible interfaces for online media," in Proceedings of CHI '99, pp. 31-32, NY: ACM, 1999.

[237] B. Ullmer and H. Ishii, "Emerging frameworks for tangible user interfaces," IBM Systems Journal, vol. 39, no. 3-4, pp. 915-931, July 2000.

[238] B. Ullmer and H. Ishii, "Emerging frameworks for tangible user interfaces," in Human-Computer Interaction in the New Millenium, (J. M. Carroll, ed.), pp. 579-601, Addison-Wesley, 2001.

[239] B. Ullmer, H. Ishii, and R. Jacob, "Token+constraint systems for tangible interaction with digital information," ACM Transactions on ComputerHuman Interaction, vol. 12, no. 1, pp. 81-118, 2005.

[240] J. Underkoffler and H. Ishii, "Illuminating light: An optical design tool with a luminous-tangible interface," in Proceedings of CHI98, pp. 542-549, NY: ACM, 1998.

[241] J. Underkoffler and H. Ishii, "Urp: A luminous-tangible workbench for urban planning and design," in Proceedings of CHI '99, pp. 386-393, NY: ACM, 1999.

[242] E. van den Hoven and B. Eggen, "Tangible computing in everyday life: Extending current frameworks for tangible user interfaces with personal objects," in Proceedings of EUSAI 2004, pp. 230-242, Springer, LNCS 3295, 2004.

[243] E. van den Hoven, J. Frens, D. Aliakseyeu, J. B. Martens, K. Overbeeke, and P. Peters, "Design Research and Tangible Interaction," Proceedings of TEI'0\%, pp. 109-115, 2007.

[244] E. van Loenen, T. Bergman, V. Buil, K. van Gelder, M. Groten, G. Hollemans, J. Hoonhout, T. Lashina, and S. van de Wijdeven, "EnterTaible: A solution for social gaming experiences," in Tangible Play: Research and Design for Tangible and Tabletop Games, Workshop at the 2007 Intelligent User Interfaces Conference, pp. S. 16-19, Honolulu, Hawaii, USA, 2007.

[245] C. L. Vaughan, "Understanding movement," in Proceedings of CHI 97, NY: ACM, 1997.

[246] R. Vertegaal and I. Poupyrev, "Introduction to the special issue on Organic user interfaces," Communications of the ACM, vol. 51, no. 6, pp. 26-30, 2008. 
[247] N. Villar and H. Gellersen, "A malleable control structure for softwired user interfaces," in Proceedings of TEI '07, pp. 49-56, NY: ACM, 2007.

[248] M. Virnes, E. Sutinen, and E. Kärnä-Lin, "How childrens individual needs challenge the design of educational robotics," in Proceedings of IDC 2008, pp. 274-281, NY: ACM, 2008.

[249] R. Want, K. Fishkin, A. Gujar, and B. Harrison, "Bridging physical and virtual worlds with electronic tags," in Proceedings of CHI99, pp. 370-377, NY: ACM, 1999.

[250] G. Weinberg and S. Gan, "The squeezables: Toward an expressive and interdependent multi-player musical instrument," Computer Music Journal, vol. 25, no. 2, pp. 37-45, 2001.

[251] M. Weiser, "Some computer science issues in ubiquitous computing," Communications of the ACM, vol. 36, no. 7, pp. 74-84, 1993.

[252] M. P. Weller, E. Y. Do, and M. D. Gross, "Posey: Instrumenting a poseable hub and strut construction toy," in Proceedings of TEI '08, pp. 39-46, NY: ACM, 2008.

[253] P. Wellner, W. Mackay, and R. Gold, "Computer-augmented environments. Back to the real world," Communications of the ACM, vol. 36, no. 7, pp. 24-26, 1993.

[254] S. A. Wensveen, J. P. Djajadiningrat, and C. J. Overbeeke, "Interaction frogger: A design framework to couple action and function through feedback and feedforward," in Proceedings of DIS '04, pp. 177-184, NY: ACM, 2004.

[255] J. Werner, R. Wettach, and E. Hornecker, "United-pulse: Feeling your partners pulse," in Proceedings of MobileHCI 2008, pp. 535-553, NY: ACM, 2008.

[256] T. Westeyn, J. Kientz, T. Starner, and G. Abowd, "Designing toys with automatic play characterization for supporting the assessment of a childs development," in Proceedings of IDC08, pp. 89-92, NY: ACM, 2008.

[257] F. R. Wilson, The Hand - How Its Use Shapes the Brain, Language, and Human Culture. Vintagebooks/Random House, 1998.

[258] P. Wyeth and H. C. Purchase, "Tangible programming elements for young children," in Proceedings of CHI '02 Extended Abstracts, pp. 774-775, NY: ACM, 2002.

[259] R. Young, D. Pezzutti, S. Pill, and R. Sharp, "The development of tools to assist the design of motion in system operated products," in Proceedings of Design and Semantics of Form and Movement - DesForM 2005, pp. 13-22, Koninklijke Philips Electronics N.V, Eindhoven, 2005.

[260] R. Young, D. Pezzutti, S. Pill, and R. Sharp, "The language of motion in industrial design," in Proceedings of Design and Semantics of Form and Movement - DesForM 2005, pp. 6-12, Koninklijke Philips Electronics N.V., Eindhoven, 2005.

[261] J. Zhang, "The nature of external representations in problem solving," Cognitive Science, vol. 21, pp. 179-217, 1997.

[262] J. Zhang and D. A. Norman, "Representations in distributed cognitive tasks," Cognitive Science, vol. 18, no. 1, pp. 87-122, 1994.

[263] Z. Zhou, A. D. Cheok, T. Chan, J. H. Pan, and Y. Li, "Interactive entertainment systems using tangible cubes," in Proceedings of IE2004, Australian Workshop on Interactive Entertainment, 2004. 
[264] J. Zigelbaum, M. Horn, O. Shaer, and R. Jacob, "The tangible video editor: Collaborative video editing with active tokens," in Proceedings of TEI " 0 \%, pp. 43-46, NY: ACM, 2007.

[265] J. Zigelbaum, A. Kumpf, A. Vazquez, and H. Ishii, Slurp: Tangibility, spatiality, and an eyedropper. CHI Extended Abstracts pp. 2565-2574, NY: ACM, 2008.

[266] O. Zuckerman, S. Arida, and M. Resnick, "Extending tangible interfaces for education: Digital montessori-inspired manipulatives," in Proceedings of $\mathrm{CHI}$ '05, pp. 859-868, NY: ACM, 2005.

[267] G. Zufferey, P. Jermann, A. Lucchi, and P. Dillenbourg, "TinkerSheets: Using paper forms to control and visualize tangible simulations," in Proceedings of TEI09, pp. 377-384, NY: ACM, 2009. 